\title{
Interactions of the Ebola virus glycoprotein with host cell factors during viral entry and release
}

\author{
Dissertation \\ for the award of the degree \\ "Doctor of Philosophy" \\ Division of Mathematics and Natural Sciences \\ of the Georg-August-Universität Göttingen
}

within the doctoral program Emerging Infectious Diseases (EIDIS) of the Georg-August University School of Science (GAUSS)

submitted by

Mariana González Hernández

from Mexico City, Mexico

Göttingen 2019 


\section{Thesis Committee}

Prof. Dr. Stefan Pöhlmann

Infection Biology Unit, German Primate Center

Prof. Dr. Lutz Walter

Department of Primate Genetics, German Primate Center

Prof. Dr. Stephan Becker

Institute for Virology, Philipps-Universität Marburg

Members of the Examination Board

Referee: Prof. Dr. Stefan Pöhlmann

Infection Biology Unit, German Primate Center

$2^{\text {nd }}$ Referee: Prof. Dr. Lutz Walter

Department of Primate Genetics, German Primate Center

\section{Further members of the Examination Board}

Prof. Dr. Uwe Gross

Department of Medical Microbiology, Institute for Medical Microbiology, University Medical Center Göttingen

Dr. Alexander Hahn

Junior Research Group Herpesviruses, German Primate Center

Dr. Jens Gruber

Medical RNA Biology, German Primate Center

Prof. Dr. Rüdiger Behr

Platform Degenerative Diseases, German Primate Center

Date of oral examination: 18.03.2019 


\section{Affidavit}

I hereby declare, that my doctoral thesis entitled "Interactions of the Ebola virus glycoprotein with host cell factors during viral entry and release" has been written independently with no other sources than quoted.

Göttingen, February 2019

Mariana González Hernández 


\section{Index}

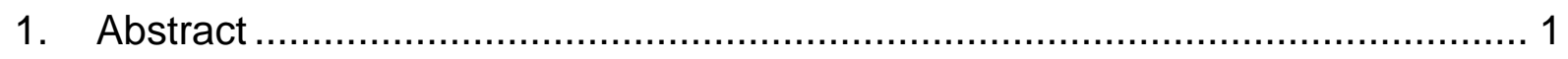

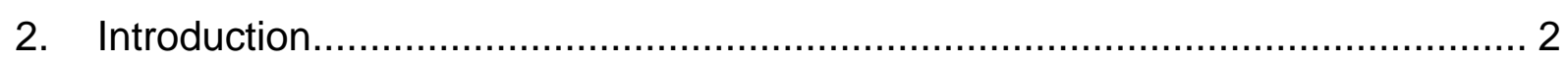

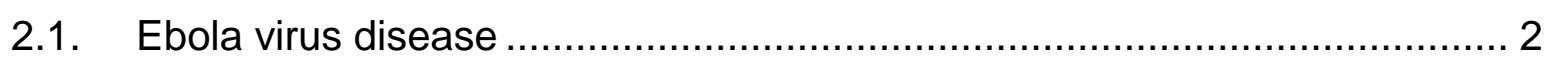

2.1.1. Ebola virus disease outbreaks ........................................................... 5

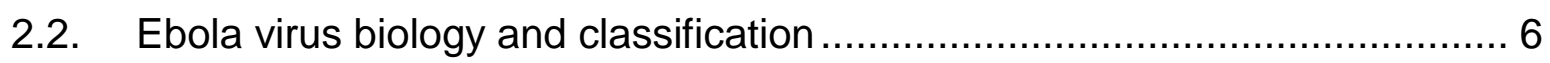

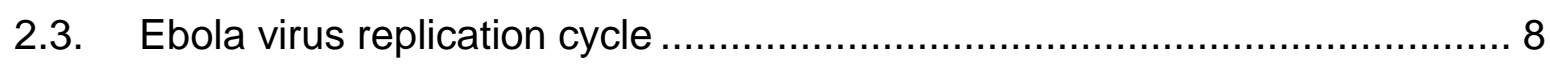

2.4. Ebola virus glycoprotein ............................................................... 10

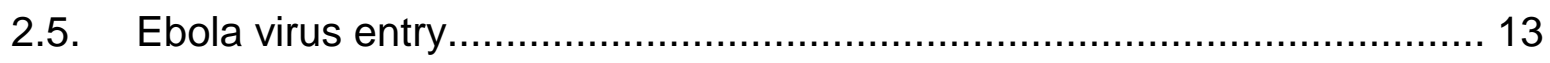

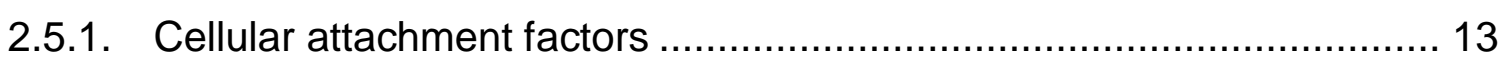

2.5.1.1. Phosphatidylserine-mediated virus entry enhancing receptors ...... 13

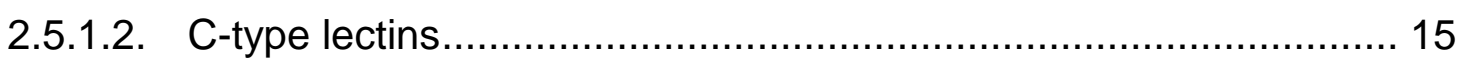

2.5.2. Virus internalization and processing of EBOV-GP …........................ 17

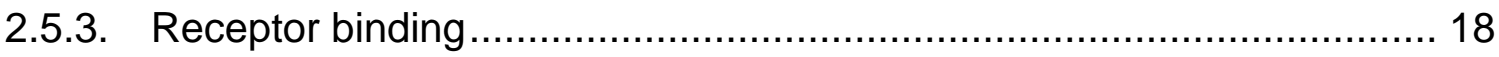

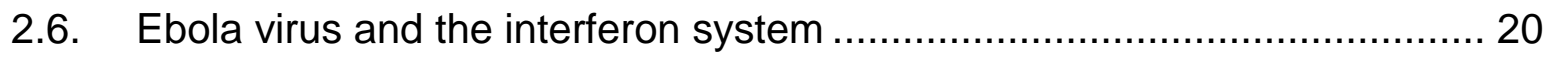

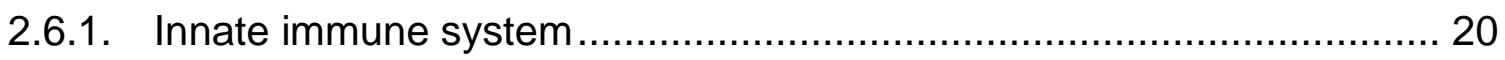

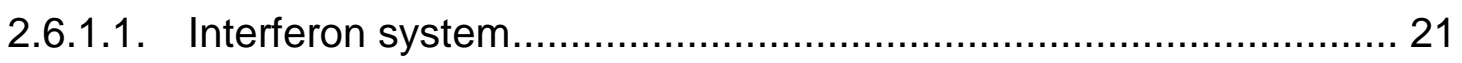

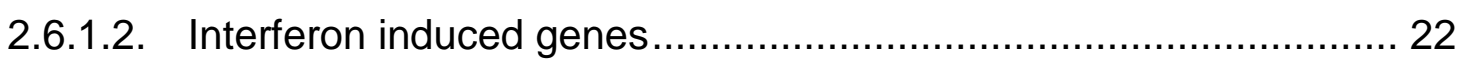

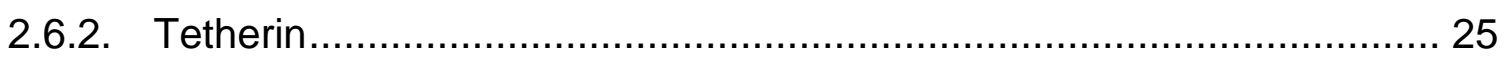

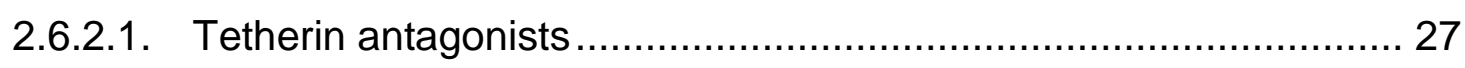

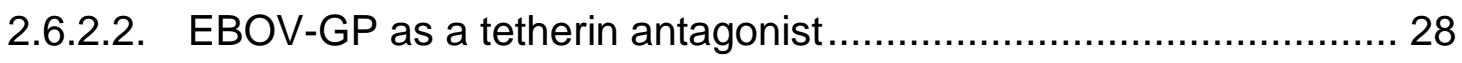

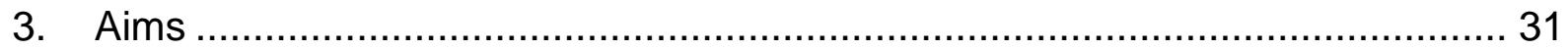

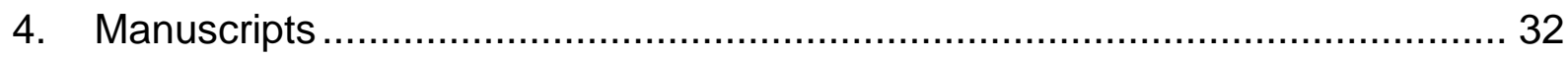

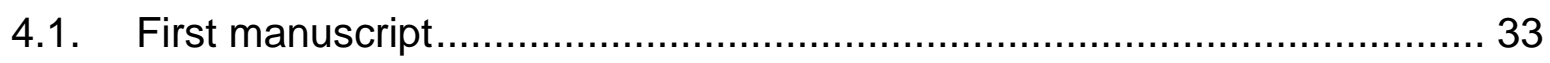

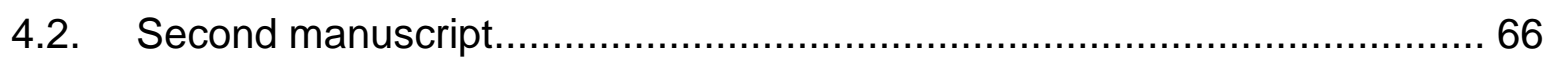

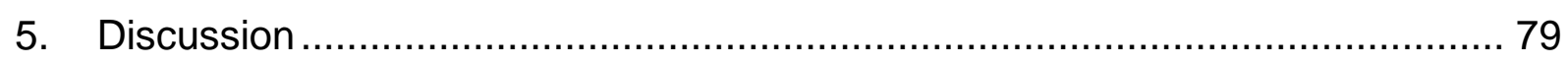

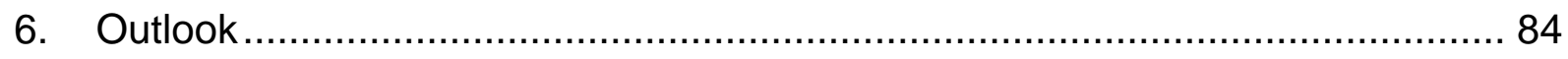

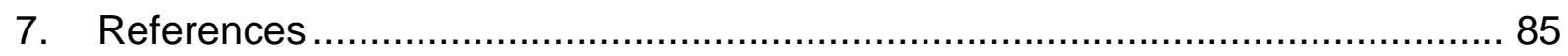

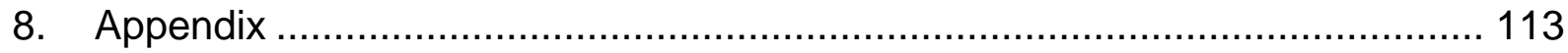

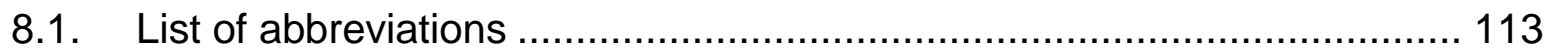

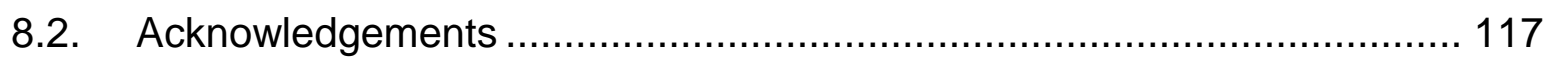

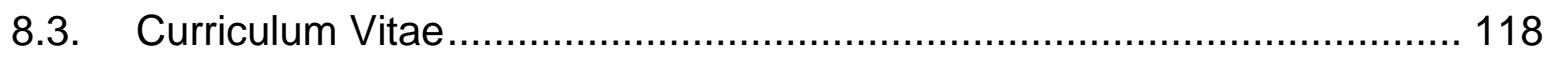

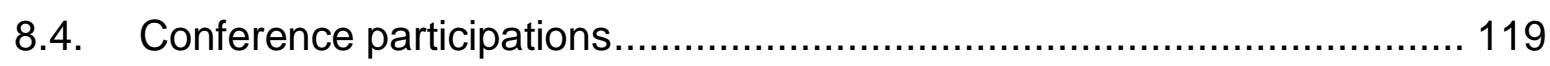

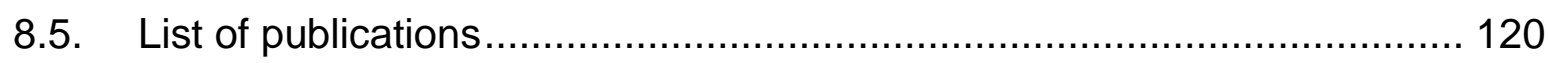




\section{Abstract}

Ebola virus disease (EVD) is a severe disease that affects humans and non-human primates. The recent EVD epidemic in West Africa shows that EVD poses a severe threat to human health. EVD is caused by Ebola virus (EBOV) and other ebolaviruses, which belong to the family Filoviridae. The EBOV glycoprotein (GP) is the only viral surface protein and mediates host cell entry. Processing of GP by host cell proteases (priming) is required for viral entry into target cells and cathepsin (Cat) $B$ and $L$ have been implicated in GP priming in cell culture. However, these enzymes may be dispensable for viral spread in the infected host and the determinants governing CatB/L dependence of viral entry are incompletely understood. Therefore, the first goal of this thesis was to identify such determinants. Apart from mediating viral entry, EBOV-GP also promotes viral release by antagonizing the interferon-induced antiviral host cell protein tetherin. However, the domains in GP that govern counteraction of tetherin and the contribution of this process to viral spread are incompletely understood. The second aim of the present thesis was thus to identify GP domains and amino acid motifs that control tetherin antagonism.

The results of the present thesis show that EBOV-GP-driven entry depends on CatB/L activity irrespective of the shape of the viral particle and the target cell type. Moreover, Calu-3, a human cell line with low endogenous CatL expression, was found to be largely resistant to entry driven by EBOV-GP and other filovirus GPs. Finally, entry was restored by directed expression of CatL or the attachment promoting factor DC-SIGN. Regarding tetherin counteraction by GP, the results obtained show that a GXXXA motif in the transmembrane domain of GP is largely dispensable for GP expression, particle incorporation and host cell entry but is required for tetherin antagonism. Lack of tetherin antagonism was observed in transfected cells and was confirmed in the context of an infectious vesicular stomatitis virus chimera encoding EBOV-GP. In summary, the present thesis identifies Calu-3 cells as one of the few cell lines largely resistant to filovirus GP-driven entry and shows that entry is limited at the stage of attachment and GP priming. Moreover, the results identify a GXXXA motif in GP as essential for tetherin antagonism and provide the first evidence that antagonism can promote viral spread, at least in the context of a surrogate system. 


\section{Introduction}

Ebola virus disease (EVD) is a severe, frequently fatal disease that affects human and non-human primates (NHP). It is caused by ebolaviruses, is highly contagious and associated with a high case-fatality rate. The EVD epidemic in West Africa from 2013 to 2016 was the largest outbreak ever witnessed and caused more cases and deaths than all previous outbreaks combined. Furthermore, secondary cases outside Africa occurred [1]. This highlights that EVD poses a severe global threat to human health

\subsection{Ebola virus disease}

Ebola virus disease is a zoonotic disease which was first described in 1976 [2]. The main causative agent of EVD is Ebola virus (EBOV), which belongs to the family Filoviridae. It has been suggested that fruit bats are the natural reservoir of EBOV. Thus, serological analyses have shown that these animals harbor EBOV-specific antibodies or viral RNA [3,4]. However, infectious EBOV has so far not been isolated from bats. This is in contrast to other filoviruses like Marburg virus (MARV), for which infectious virus has been isolated from fruit bats [5-8]. Thus, fruit bats are most likely the reservoir of EBOV but contribution of other animals to maintenance in nature cannot be excluded $[9,10]$.

Transmission of EBOV to humans occurs via contact with body fluids or organs of infected wild animals such as chimpanzees, gorillas, fruit bats, monkeys, or forest antelopes [11,12]. Once the virus has been introduced into the human population, human-to-human transmission may occur via direct contact with body fluids from infected patients or deceased people. In addition, recent studies suggest that EBOV can be sexually transmitted and that transmission can occur for up to 12 months after recovery of male survivors [1,13-17].

The incubation period of EVD ranges from 2 to 21 days in humans $[2,18]$. Symptoms start abruptly with fever, muscle pain, cough, headache and abdominal pain. During the next phase of the disease symptoms like vomiting, diarrhea, dyspnea, hypovolemic shock, and organ failure occur [19-21]. Studies conducted during the outbreak in West Africa revealed that hemorrhages occur in approximately $5 \%$ of the patients but are 
not associated with fatal outcome [20-23]. These studies confirm results obtained during the EBOV outbreaks in Kikwit 1995 [24] and Uganda 2000 [25]. Instead, hypovolemic shock and organ failure lead to death usually within 7 to 14 days after the onset of the symptoms $[20,21,26]$. The adequate supportive care, like administration of intravenous fluids, control of electrolytes, and prevention of shock associated complications, increases the chances of survival [21,26]. During the West African outbreak persistence of EBOV in immune-privileged sites (i.e. testicles [14,27], eye [28], central nervous system [29], and breast milk [30]) of EVD survivors was recorded. Moreover, persistently infected convalescent patients transmitted the virus to others raising the possibility that EVD might become endemic in the African population [31].

Vaccination against EBOV. To date, there are no approved vaccines or antiviral agents to combat EVD. However, several pre- and post-exposure treatments for EVD are under development, including vaccines based on inactivated EBOV, recombinant viruses, virus-like particles, DNA and plant-based antibodies. The first attempt to develop a vaccine against EVD was focused on heat- or formalin-inactivated EBOV preparations. Both showed protection in a guinea pig model. However, incomplete inactivation is always a concern [32,33]. In another trial, virus-like particles (VLPS), based on the EBOV glycoprotein (GP), viral protein (VP) 40 and nucleoprotein (NP), were protective in rodent models [34]. The most promising results in vaccine development have been obtained with recombinant viral vectors. Among these, the replication-competent vesicular stomatitis virus-EBOV chimera (VSV-EBOV), which harbors the gene for EBOV-GP, has been used in clinical trials which revealed that it is well tolerated when used in low doses $[35,36]$. However, when higher vaccine doses were applied, vaccinated volunteers developed arthralgia and arthritis [35-38]. Notably, VSV-EBOV conferred up to $100 \%$ protection in NHP models [39] and was found to be highly efficient in preventing EVD in humans [38] as demonstrated during the epidemic in West Africa. Moreover, it is being used in the recent outbreak in the Democratic Republic of Congo (DRC) [40,41]. However, it has not been approved by regulatory agencies so far and is still in phase III clinical trials $[42,43]$. The vaccine GamEvac-Combi has been tested in clinical trials in Russia. This vaccine contains two EBOV-GP expression systems: live attenuated recombinant VSV and a recombinant replication defective adenovirus serotype-5 (Ad5) [44]. During the clinical trial 
vaccinated patients did not show serious adverse effects and strong humoral and cellular immune response against EBOV-GP were detected [44]. Furthermore, vaccination with VSV-EBOV-prime Ad5-boost vaccine induced a significantly stronger antibody response than immunization with VSV-EBOV alone [44-46].

EVD treatment. Passive immunization using whole blood, plasma or serum from EVD survivors, is a treatment option recommended by the World Health Organization (WHO) in emergency conditions. Unfortunately, studies in NHP and in EVD patients showed no association of treatment with survival [47-53]. Therefore, application of purified antibodies is a preferable therapeutic option. Antibody cocktails such as ZMapp, ZMab or MIL77 confer full protection against EBOV infection in NHP. For instance, studies carried out during the 2013-2016 epidemic suggest that ZMapp improves survival of EVD patients [54]. Accordingly, the humanized version of ZMapp antibodies has been recommended as EVD treatment by WHO [55,56]. Another monoclonal antibody that has been under study is $K Z 52$, a neutralizing antibody obtained from an EVD survivor [57]. This antibody prevents EBOV-GP interaction with host cell proteases and protected guinea pigs against EBOV challenge, but failed to protect rhesus macaques [57-59]. Despite the largely encouraging results discussed above, efficiency and cross-reactivity of the monoclonal antibodies with other ebolavirus species need to be improved.

For post-exposure treatment antiviral agents that can inhibit viral replication are available. Favipiravir (T-705), a drug first developed for influenza treatment, was found to inhibit the RNA polymerase of EBOV in cell culture and was administrated to humans during the EVD epidemic in West Africa. However, treatment had only a weak effect on viral replication and was not associated with a survival benefit [60]. Several other small molecules are currently under study for their possible use in EVD treatment. Interferons (IFN) are natural antivirals and IFN-y has been approved by the Food and Drug Administration as a treatment for chronic diseases, such as Chronic Granulomatous Disease and osteopetrosis [61]. Furthermore, IFN- $\gamma$ treatment can reduce the mortality rate in mice when administrated before or after EBOV challenge [62]. These results indicate that IFNs could be repurposed and adapted as a prophylactic treatment of EVD $[41,62]$. Toremifene and clomiphene can block EBOV 
entry into target cells and exert antiviral activity in cell culture and in mice models $[63,64]$. Nafamostat mesylate is a protease inhibitor that reduces cathepsin (Cat) $B$ levels in rat pancreas [65] and for EBOV entry the viral GP needs to be processed by CatB. Therefore, Nafamostat might be suitable for EVD treatment but its antiviral activity has not been assessed in animal models [66]. Another strategy to treat EVD are small interfering RNAs (siRNAs) that target viral mRNAs encoding for proteins essential for EBOV replication and assembly, such as the RNA polymerase (L), and the viral proteins VP35 and VP24. A study showed that a combination of siRNAs targeting these three proteins can provide post exposure protection against EBOV challenge in a NHP model [41]. In sum, treatment options against EVD are available. However, efficiency and activity against diverse ebolavirus species need to be improved.

\subsubsection{Ebola virus disease outbreaks}

The first recorded EVD outbreaks occurred in 1976 in the DRC (formerly Zaire), and in Sudan and were caused by members of two different ebolavirus species, EBOV (species Zaire ebolavirus) and Sudan virus (SUDV, species Sudan ebolavirus), respectively. Since then, several EVD outbreaks caused by EBOV (most frequently), SUDV, Tai Forest virus (TAFV, species Taï Forest ebolavirus), and Bundibugyo virus (BDBV, species Bundibugyo ebolavirus) have been recorded in remote areas within Central Africa and although case-fatality ratios were high, the case numbers were limited to a maximum several hundred per outbreak $[1,18]$.

In March 2014, the WHO reported an EVD outbreak in Guinea that was caused by EBOV. The outbreak started in December 2013 and then turned into an epidemic that spread to Sierra Leone and Liberia. It was declared a Public Health Emergency of International Concern (PHEIC) in August 2014. During the 2013-2016 EVD epidemic in West Africa, the virus reached densely populated areas, partially due to a delayed response of local authorities and WHO. Furthermore, secondary cases outside Africa (Spain and the United States of America) were recorded. By the time the WHO lifted the PHEIC in March 2016, there were more than 28,000 cases and 11,000 deaths reported, which are more cases than in all previous outbreaks combined. In June 2016, the WHO declared the end of EVD transmission in Guinea and Liberia $[18,67]$. 
After the West African epidemic, a small EVD outbreak in the DRC was registered in May 2017 and declared over in July 2017. During this outbreak five confirmed EVD and three probable cases have been reported, four of which died [68]. An ongoing EVD outbreak in the DRC, again caused by EBOV, started in August 2018 and until January $22^{\text {th }}, 2019$ there were 713 cases (664 laboratory confirmed cases by RT-PCR, positive IgM or virus isolation) and 439 deaths reported. So far, only north-eastern provinces of DRC including North Kivu and Ituri have been affected, but current outbreak hotspots (Butembo, Katwa) encompass an urban area with a population of roughly one million people. Containing the current outbreak has been challenging due to security conflicts, a long-term humanitarian crisis and other ongoing epidemics in the area. Currently, WHO's risk assessment is high at regional and national levels $[18,69]$.

\subsection{Ebola virus biology and classification}

Ebola virus belongs to the family Filoviridae, which comprises three genera: Ebolavirus, Marburgvirus and Cuevavirus. According to the International Committee on Taxonomy of Viruses there are five species in the genus Ebolavirus: Zaire ebolavirus (single member: Ebola virus, EBOV), Sudan ebolavirus (single member: Sudan virus, SUDV), Taï Forest ebolavirus (single member: Tai Forest virus, TAFV), Bundibugyo ebolavirus (single member: Bundibugyo virus, BDBV) and Reston ebolavirus (single member: Reston virus, RESTV) [70]. All members of the genus Ebolavirus are highly pathogenic for humans, except for RESTV. A recent study discovered a sixth species, Bombali ebolavirus (single member: Bombali virus, BOMV) [6]. BOMV has only been detected in bats but the viral glycoprotein can mediate entry into human cells. However, no human infections were documented so far. The genus Marburgvirus contains only one species Marburg marburgvirus (two members: Marburg virus, MARV; and Ravn virus, RAVV) [71], both are known to be pathogenic in humans, and MARV outbreaks have been reported in the past [72]. Lloviu cuevavirus (single member: Lloviu virus, LLOV) is the only species within the Cuevavirus genus, but no human infections have been reported so far [5].

Filovirus particles are filamentous (Figure 1A), with a fixed diameter of $80 \mathrm{~nm}$ and a variable length (up to $14,000 \mathrm{~nm}$ ). They are enveloped and contain a negative sense 
single-stranded RNA (ssRNA) genome of approximately $19 \mathrm{~kb}$. EBOV is the most studied member within the genus Ebolavirus, consequently the subsequent discussion will focus on EBOV. The EBOV genome consists of seven open reading frames (ORFs) that encode for seven structural proteins: nucleoprotein (NP), viral protein $35,40,30$, 24 (VP35; VP40; VP30; VP24), RNA polymerase (L), and glycoprotein (GP) (Figure 1B) [73-75]. NP encapsidates the viral genome, jointly with VP30, which acts as a transcriptional activator. The polymerase $L$ is responsible for viral genome transcription and replication $[76,77]$. VP35 is a polymerase cofactor and along with NP, VP30 and $\mathrm{L}$, forms the ribonucleocapsid. VP24 is required for nucleocapsid maturation [77-79]. VP40 forms the matrix of the virus and is responsible for virus assembly and budding $[80,81]$. Finally, GP is incorporated into the viral membrane and drives viral entry into target cells [82].

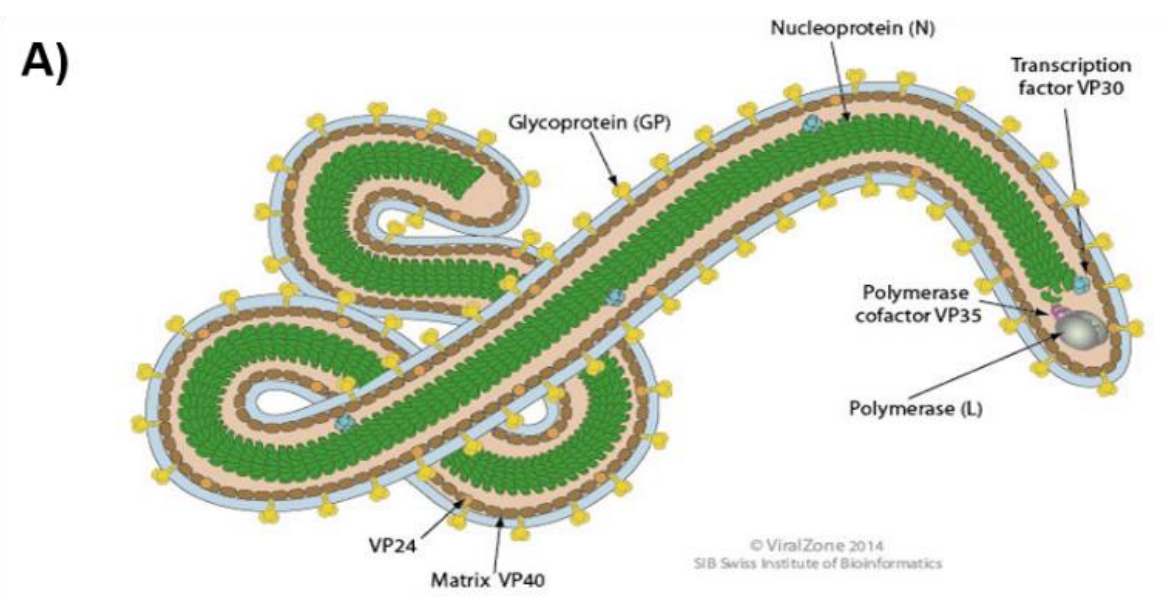

B)

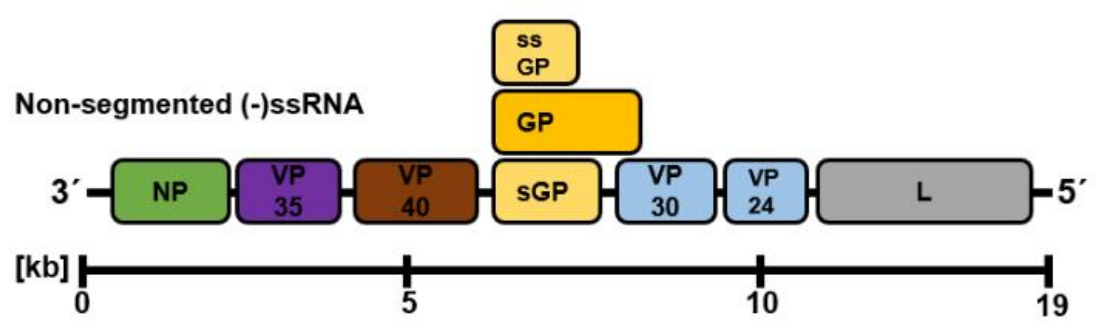

Figure 1. Particle architecture and genome organization of Ebola virus. A) EBOV particles are filamentous; GP is the only viral surface protein. VP35 is a polymerase cofactor and along with NP, VP30 and L, forms the ribonucleocapsid. VP40 forms the viral matrix along with VP24. B) Schematic representation of EBOV genome organization and the encoded proteins. (Adapted from ViralZone [83]) 


\subsection{Ebola virus replication cycle}

EBOV and other filoviruses replicate in the cytoplasm. First, EBOV-GP attaches to host cell surface factors and viral particles are internalized via macropinocytosis [84]. Subsequently, virions are trafficked to late endosomes where GP is processed (primed) by the cellular cysteine proteases CatB/ L. Primed EBOV-GP then interacts with the intracellular receptor Niemann-Pick C1 (NPC1) $[85,86]$. Engagement of NPC1 and a subsequent poorly defined stimulus triggers GP-driven fusion of the viral envelope with the endosomal membrane [87-89]. This requires large conformational changes in GP that are also observed with unrelated viral glycoproteins, collectively referred to a class I membrane fusion proteins [90]. In the course of these conformational rearrangements a fusion loop in GP2 is inserted into the host membrane and a thermostable six-helix bundle structure in GP2 is formed, which results in close approximation of the viral and the cellular membrane. Finally, a fusion pore is formed which allows the release of the nucleocapsid into the host cytoplasm [91-97]. Then, the viral polymerase L produces mRNAs and cRNA that will serve as template for generation of new genomic (-)ssRNA [98]. New viral ribonucleoprotein complexes (RNP) are assembled in the perinuclear region and, stimulated by VP40, transported to the budding sites via actin-dependent RNP trafficking [99,100]. GP is transported via the endoplasmic reticulum (ER) and Golgi apparatus to the site of viral budding where it colocalizes with VP40 in cholesterol-enriched microdomains [101103]. At the plasma membrane, VP40 forms hexamers and facilitates the incorporation of the RNP into filamentous particles. In order to facilitate release, VP40 recruits cellular proteins including Nedd4/Rsp5, Tsg101 and Vps4 [104-106], which induce translocation of VP40 to the phosphatidylserine-enriched inner leaflet of the plasma membrane and catalyze fission of the plasma membrane during budding [107] (Figure 2). 


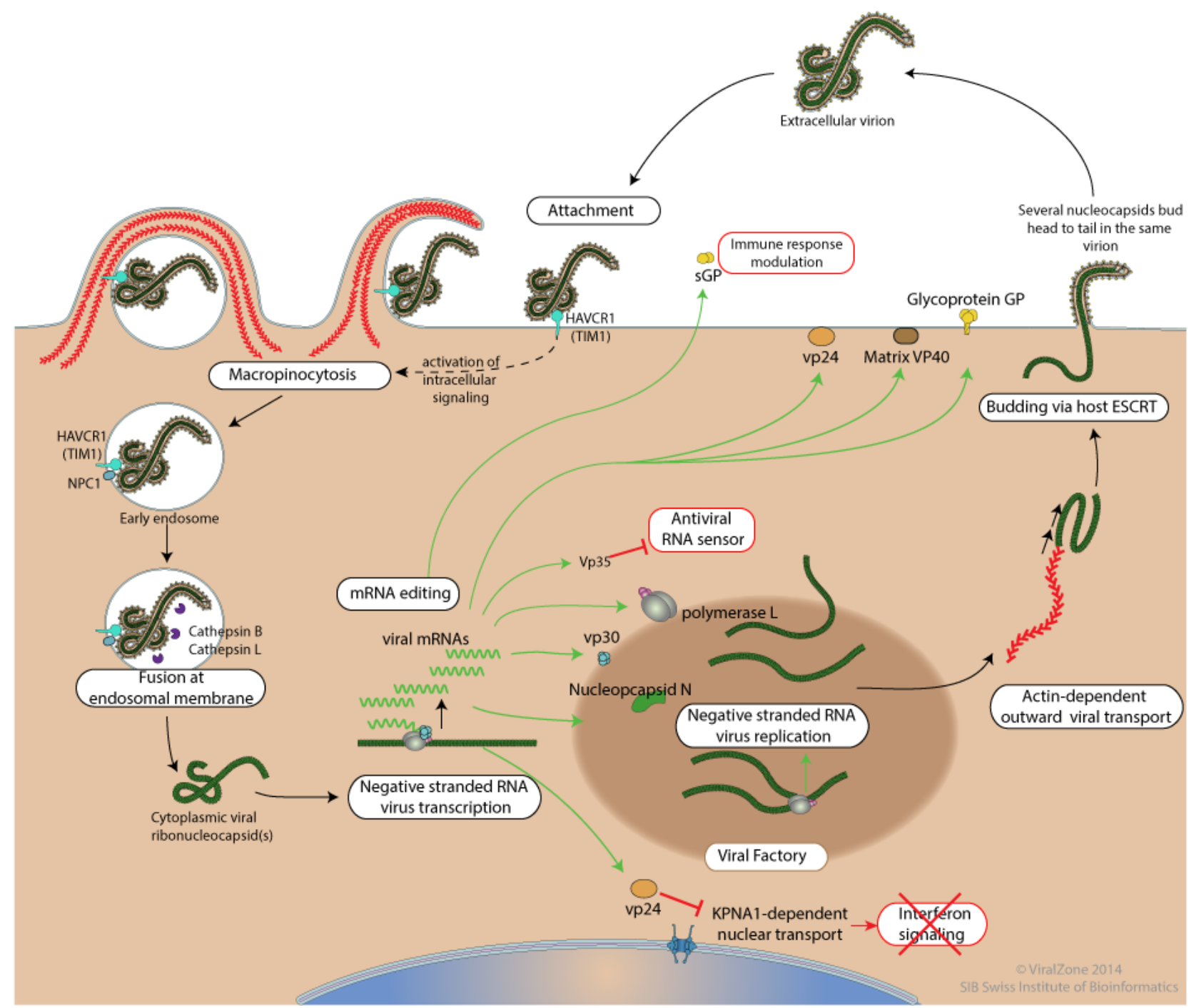

Figure 2. Ebola virus replication cycle. (Reprinted from [83]) 


\subsection{Ebola virus glycoprotein}

The GP gene is the only gene of EBOV able to encode for different proteins. It can produce three mRNAs encoding for pre-soluble GP, pre-GP $\left(\mathrm{GP}_{0}\right)$ and pre-small soluble GP proteins in approximately 14:5:1 ratio, although this ratio can be cell type dependent (Figure 3) [76,108].
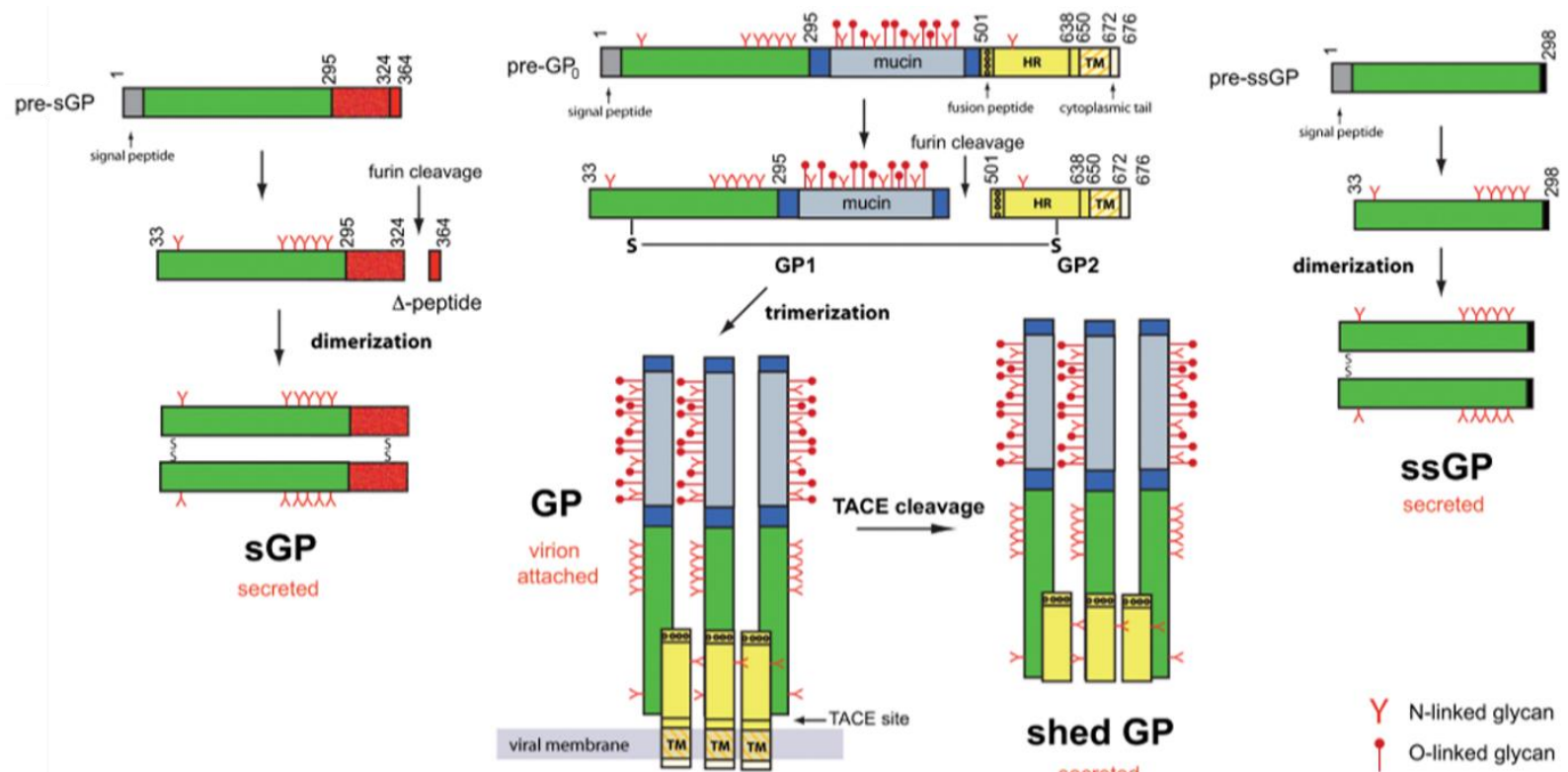

dimerization
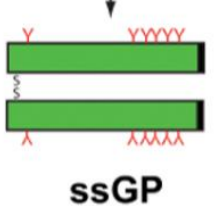

secreted

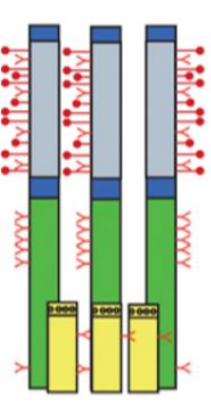

shed GP

secreted

$Y$ N-linked glycan

- O-linked glycan

Figure 3. Proteins encoded by the GP open reading frame. Pre-sGP (left), is encoded by the primary transcript and is most abundant. Processing of pre-sGP leads to formation of mature $\mathrm{N}$-glycosylated dimers of sGP and O-glycosylated monomers of $\Delta$-peptide. The mRNA encoding for

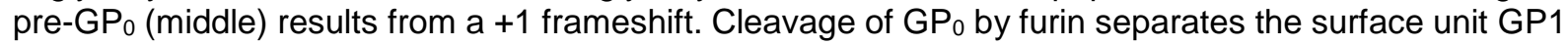
from the transmembrane unit GP2, which remain associated by a disulfide bond. GP trimers are incorporated into the viral membrane that decorate the viral surface. Cleavage of GP by ADAM17/TACE results in the production of shed GP. Pre-ssGP (right) is encoded by a +2 frameshift in the GP ORF and is the least abundant transcript. Mature ssGP is secreted as N-glycosylated dimer. (Adapted from [109])

The soluble GP (sGP) is encoded by unedited mRNA and is the most abundant product of the GP gene. It is synthesized as pre-sGP and post-translational modifications include proteolytic cleavage by furin resulting in two products, sGP and delta-peptide ( $\Delta$-peptide). Both proteins are secreted due to their lack of transmembrane domains [110]. Mature SGP is released extracellularly as $\mathrm{N}$-glycosylated dimer [111,112]. The role that SGP plays in EBOV pathogenesis is not completely understood. Since it shares the N-terminal 295 aa with full-length GP, it has been suggested that sGP acts as a decoy antigen for antibodies directed against full-length GP [101]. Furthermore, it has been postulated that it can lead to antigenic subversion, i.e. high amounts of sGP divert the immune response away from full-length GP by acting as a target for neutralizing antibodies [113]. Studies performed in cell culture have shown that sGP 
may exert anti-inflammatory activity, such as restoration of the barrier function of endothelial cells, and inactivation of neutrophils [114,115]. Finally, it has been suggested that SGP could reduce viral cytotoxicity by limiting the expression of EBOV-GP $[116,117]$.

The $\Delta$-peptide is the C-terminal cleavage product of the pre-sGP protein and is O-glycosylated [110]. It has been proposed that $\Delta$-peptide may prevent virus superinfection by binding to permissive cells and interfering with viral entry [118]. However, the in vivo relevance of this process has not yet been determined. Another hypothesis, based on in silico analysis of $\Delta$-peptide sequences, suggests that it acts as a viroporin, but experimental evidence is lacking [119].

The small soluble protein (ssGP) is produced as the result of a +2 frameshift in the GP ORF [76,120]. It is largely $\mathrm{N}$-glycosylated and secreted as dimers. Despite having similar biochemical properties and primary sequence as sGP, ssGP does not exhibit the same anti-inflammatory function in endothelial cells as sGP. To date, ssGP function and its contribution to EBOV pathogenesis are still unknown [108,121].

The full-length glycoprotein (GP) is the only viral surface protein. It is synthesized as a precursor $\left(\mathrm{GP}_{0}\right)$ as the result of $a+1$ frameshift of the GP ORF [76,120]. GPo post-translational modifications start in the ER where it is $\mathrm{N}$ - and O-glycosylated. Then, it is transported into the Golgi apparatus where furin cleavage divides it into two subunits, the surface unit GP1 and the transmembrane unit GP2, remain associated via a disulfide bond between GP1 Cys53 and GP2 Cys609 [102,122]. The GP1-GP2 heterodimers assemble into trimers that are transported to the cell surface where they are incorporated into in the viral envelope [109,123]. The GP1 subunit contains the signal peptide, the receptor binding domain (RBD), and a heavily $\mathrm{N}$ - and O-glycosylated mucin-like domain (MLD) that is rich in serine, threonine and proline residues. The GP2 subunit comprises an internal fusion loop, two heptad repeat regions (HR1, HR2), a transmembrane domain (TMD) and a short cytoplasmic tail $[93,102]$. A schematic representation of EBOV-GP domains and trimeric structure is depicted in figure 4. Additionally, EBOV-GP located at the plasma membrane can be cleaved by tumor necrosis factor alpha converting enzyme (TACE), a member of the 
disintegrin and metalloproteinase family, resulting in release of shed GP [124]. As well as sGP, shed GP can act as an antibody decoy capturing anti-EBOV-GP antibodies or stimulate antibody dependent enhancement of infection [124]. In addition, it has been suggested that shed GP might modulate the homeostasis of the endothelium, the secretion of pro-inflammatory cytokine $[125,126]$, and may reduce the abundance of GP on the cell surface and in virions $[117,127]$.
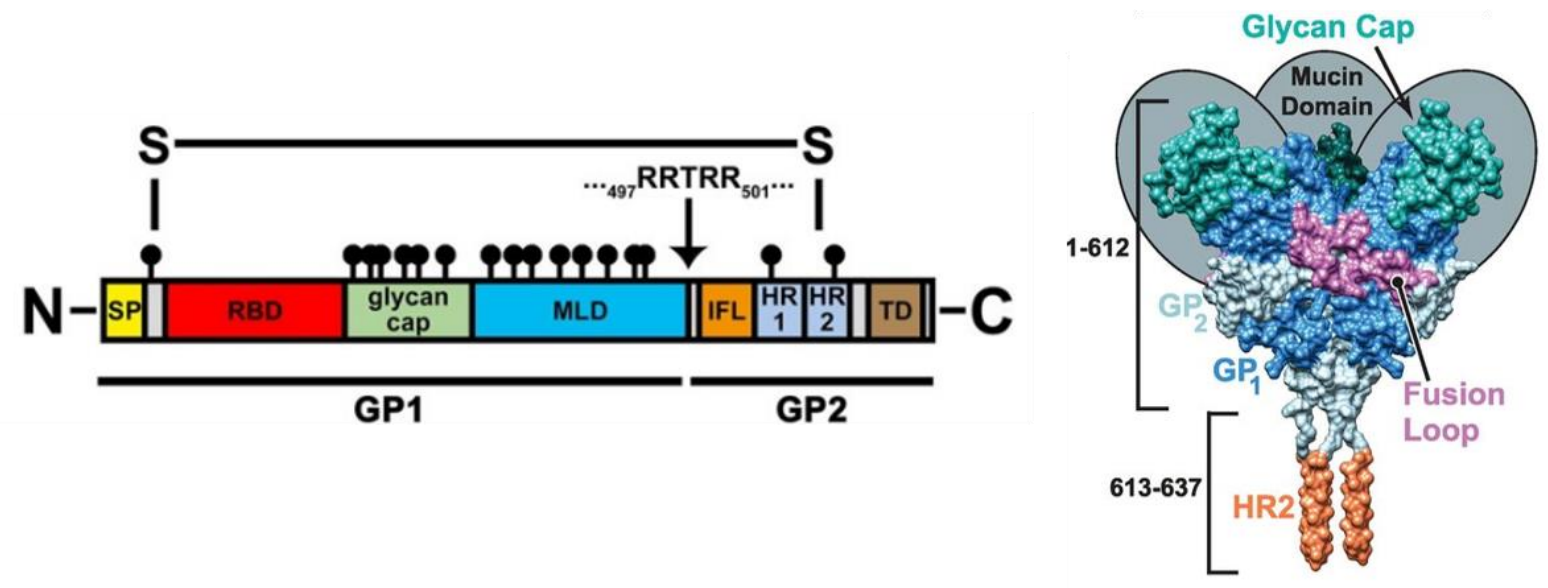

Figure 4. Ebola virus glycoprotein domain organization and 3D structure. Schematic representation of the domain organization of EBOV-GP is shown in the left panel. SP = signal peptide (yellow), RBD = receptor binding domain (red), glycan cap (green), MLD = mucin-like domain (blue), IFL $=$ internal fusion loop (orange), HR = heptad repeat 1 and 2 (light blue), TD = Transmembrane domain (brown) (Adapted from [128]). The right panel shows the domain organization of the GP in the context of the 3D structure of the protein (Adapted from [129])

Role of the Ebola virus glycoprotein in viral pathogenesis. EBOV-GP mediates viral entry into host cells, the first step in the EBOV replication cycle. Entry comprises viral attachment to the cell surface, viral uptake into cells, binding of GP to its intracellular receptor NPC1 and membrane fusion, as outlined above and as described in detail below [82,130]. Several studies have suggested that GP is also involved in EBOV pathogenesis and in the activation of the inflammatory response [115,131,132]. Expression of EBOV-GP causes cytotoxic effects, cell rounding and detachment of adherent cells in vitro. Moreover, analogous observations have been reported in explanted blood vessels, which results in increased vascular permeability [133,134]. The MLD contributes to cellular cytotoxicity by masking adhesion molecules, including intercellular cell adhesion molecule-1 (ICAM-1), platelet/endothelial cell adhesion molecule-1 (PECAM-1), vascular cell adhesion molecule-1 (VCAM-1) and epidermal growth factor receptor (EGFR) of the host cell surface [135-137]. The steric shielding not only reduces accessibility and function of adhesion molecules but also of the major 
histocompatibility complex class I, suggesting that the immune cell recruitment might be altered. Therefore, shielding might contribute to immune suppression and activation of the inflammatory response during EBOV infection [131,132,136,138,139]. Although it has been shown that the MLD plays a major role in EBOV-GP mediated cytotoxic effects, it is dispensable for EBOV-GP-driven entry [102,140]. It has been suggested that the MLD glycans cover antigenic epitopes within the RBD. This might be an immune evasion strategy mediated by the MLD since it blocks the activity of neutralizing antibodies directed against this domain [136,137,141]. Finally, sGP and shed GP might modulate immune responses and integrity of the vasculature as outlined above.

\subsection{Ebola virus entry}

Ebola virus entry can be divided in four steps: attachment, viral uptake and proteolytic priming of GP, receptor binding and membrane fusion.

\subsubsection{Cellular attachment factors}

Attachment is mediated by interactions of the GP1 subunit or phosphatidylserine (PtdSer) in the viral envelope with proteins located at the surface of host cells [142144]. Engagement of these attachment promoting factors can augment virion uptake but is not essential for EBOV-GP- driven entry

\subsubsection{Phosphatidylserine-mediated virus entry enhancing receptors}

PtdSer-mediated virus entry enhancing receptors (PVEERs) are a group of receptors and receptor complexes with the ability to bind PtdSer present on the viral envelope. The mechanism underlying augmentation of viral infectivity by PVEERs is called apoptotic mimicry. It was first postulated to be used by hepatitis B virus and was first experimentally confirmed in the context of vaccinia virus infection [145,146]. PVEERs bind to PtdSer, which is expressed on the inner leaflet of the plasma membrane of living cells. However, PtdSer is exposed on the surface of apoptotic or necrotic cells, allowing phagocytic cells to recognize and remove these cells [145,147]. PtdSer binding to PVEERs can occur through direct interaction or through a ligand that can bind both PtdSer and the receptor [148,149]. Several viruses, including members of the flavivirus, filoviruses, arenavirus, baculovirus and alphavirus families are known to 
take advantage of apoptotic mimicry $[143,144,149,150]$. The PVERRs used by filoviruses for attachment are TIM-1, TIM-4, Axl, Dtk and MER [143,151,152].

T-cell immunoglobulin and mucin (TIM) family. The human TIM family proteins are type I cell surface glycoproteins with an $\mathrm{N}$-terminal immunoglobulin variable (IgV)-like domain, a mucin-like domain (O-glycosylated), a transmembrane domain and a C-terminal cytoplasmic tail [153]. The ligand binding pocket of TIM-1 and TIM-4 is located between two loops of the IgV domain [154-156]. Aspartate and asparagine residues present in the upper loop coordinate calcium ions and form hydrogen bonds with the phosphate and serine groups of PtdSer [157]. Binding of PtdSer to TIM proteins is necessary for clearance of apoptotic bodies and immune cell regulation [158-162]. TIM-1 is mainly expressed on immune cells such as B cells, mast cells, Th2 $\mathrm{CD}^{+} \mathrm{T}$ cells but is also found on epithelial cells of kidney, airway and eye mucosa [163-167]. TIM-4 is expressed on macrophages and mature dendritic cells (DCs) located in spleen, lymph node, and peritoneum [154,155,168]. Macrophages and DCs are known to be early and sustained target cells during EBOV infection [169,170]. It has been demonstrated that EBOV-GP can interact with TIM-1 and TIM-4 via its receptor binding domain, further leading to virus internalization and increased infectivity [167]. However, subsequent studies showed that TIM proteins recognize PtdSer displayed on the viral envelope and thereby augment viral entry $[143,144]$.

Tyro3/AxI/Mer (TAM) family. Tyro3, Axl, and Mer (TAM) belong to the family of receptor tyrosine kinases. They possess two N-terminal immunoglobulin (lg)-like domains, followed by two fibronectin type III domains, a single transmembrane domain, and a cytoplasmic protein tyrosine kinase (PTK) domain. TAM proteins interact via their Ig-like domains with their ligands Gas6 (Axl) and protein S (Tyro3 and Mer). This interaction activates the TAM receptors and results in autophosphorylation of tyrosines in the PTK domain [171,172]. TAM proteins are involved in cytokine release, cell proliferation, survival, and adhesion [173]. Similar to TIM proteins, they bind to PtdSer exposed on apoptotic cells, and binding is facilitated by the $\mathrm{Y}$-carboxyglutaminc acid residues present in Gas6/Protein S [174,175]. PTK activity and signaling of TAM proteins is required for inhibition of inflammation and augmentation of viral entry $[149,176,177]$. TAM proteins are expressed on a broad spectrum of tissue and cells, 
e.g. lung, brain, kidney, platelets, macrophages, natural killer (NK) cells, and DCs [173,178-181] and might thus promote EBOV entry into a broad spectrum of target cells. Finally, it is noteworthy that AxI may promote EBOV host cell entry by enhancing macropinocytosis [151,182-184], and that $A x I$, unlike TIM-1, requires proper ligand binding and kinase signaling to enhance EBOV entry $[185,186]$.

The mechanism by which PtdSer is exposed on the outer leaflet of the membrane of enveloped viruses is unknown. A recent study by Nanbo and colleagues suggests that XK-related protein (Xkr) 8, a cellular scramblase responsible for exposure of PtdSer on apoptotic cells, plays an important role. Thus, Xkr8 and EBOV-GP are trafficked together to the viral budding sites and are incorporated into EBOV-like particles, and incorporation of $\mathrm{Xkr8}$ is required for augmentation of viral entry in a PtdSer-dependent manner [187].

\subsubsection{C-type lectins}

C-type lectins (CTLs) are one of the largest families of animal lectins that have been classified into 17 groups (I-XVII), based on phylogeny as well as structural and functional properties [188]. In vertebrates, CTLs functions include serum glycoprotein homeostasis, pathogen sensing, and the initiation of immune response [189]. CTLs depend on $\mathrm{Ca}^{2+}$ to recognize glycan ligands. They recognize a wide range of ligands including proteins, lipids, inorganic molecules, and ice crystals [190].

Myeloid C-type lectins. Myeloid CTLs are pattern recognition receptors (PRRs) specialized in glycolipid and glycoprotein recognition [191,192]. They are mainly expressed by antigen-presenting cells including DCs, macrophages and monocytes [189]. C-type lectin receptors (CLRs) are at the frontline of innate and adaptive antiviral immune responses, CLR-virus interaction triggers a signaling cascade that induces the production of pro-inflammatory cytokines [193,194]. Dendritic cell-specific intercellular adhesion molecule-3-grabbing non-integrin (DC-SIGN) also known as CD209, is a type II transmembrane receptor composed of a short $\mathrm{N}$-terminal cytoplasmic tail, a transmembrane region, a flexible neck domain involved in oligomerization, and a $\mathrm{Ca}^{2+}$-dependent carbohydrate recognition domain. DC-SIGN binds to high mannose glycans and fucose-containing glycans [195-197]. Several viruses, including human 
immunodeficiency virus type 1 (HIV-1), EBOV, dengue virus (DENV) and severe acute respiratory syndrome coronavirus (SARS-CoV) are recognized by DC-SIGN [198]. Lymph node-specific intercellular adhesion molecule-3-grabbing integrin (L-SIGN) also known as DC-SIGNR has a similar structure as DC-SIGN. However, it has a polymorphic neck region and the length of the DC-SIGNR neck region may impact affinity for ligands and viral pathogen specificity [193,198]. L-SIGN binds to N-linked high mannose oligosaccharides, with preference for mannosylated residues [193,198,199]. EBOV [200], MARV [201], HIV-1 [202], hepatitis C virus (HCV) [203], hepatitis B [204], and SARS-CoV [205] exploit L-SIGN for glycoprotein-mediated attachment and internalization. Liver and lymph node sinusoidal endothelial cell C-type lectin (LSECtin) recognizes glycans containing mannose, $\mathrm{N}$-acetylglucosamine and fucose residues. LSECtin can enhance EBOV and SARS-CoV but not HIV-1 or HCV infection and may do so in a mannose-independent manner [206-208]. Macrophage galactose C-type lectin (MGL) binds galactose and $\mathrm{N}$-acetylgalactosamine residues, and viruses like EBOV, MARV and influenza A virus (IAV) use it for cellular entry and for evasion of host immune responses [209-213].

Asialoglycoprotein receptors. Asialoglycoprotein receptors (ASGPRs) are $\mathrm{Ca}^{2+}$ dependent type II transmembrane proteins, with a short cytoplasmic tail, a transmembrane domain and a carbohydrate binding domain. Their main function is to regulate glycoprotein levels in serum, thereby maintaining homeostasis. ASPGR-1 is expressed in liver and has been shown to enhance MARV entry [188,214,215].

Cellular lectins such as MGL, DC-SIGN, L-SIGN, LSECtin, and ASGPR-1 can increase filovirus attachment to susceptible cells [200,207,209,214,216,217] by binding to $\mathrm{N}$ - and/or O-linked glycans on GP. However, binding to these molecules is usually not sufficient to allow for infectious entry, although one study suggested that directed expression of DC-SIGN and L-SIGN on the T cell line Jurkat renders these cells susceptible to GP-driven entry [200,216,218,219]. 


\subsubsection{Virus internalization and processing of EBOV-GP}

The next steps of EBOV entry are particle uptake and proteolytic processing of GP. Initially it was thought that EBOV and filoviruses in general were internalized in a clathrin- and/or caveolin-dependent fashion [220-222]. However recent studies showed that macropinocytosis is the main mechanism for EBOV uptake and it depends on particle size, since particles bigger than $100 \mathrm{~nm}$ are usually internalized via macropinocytosis. Furthermore, it was shown that EBOV induces PI3K, Rac1, PKC, Cdc42 and Pak1 activity and triggers plasma membrane ruffling, which are factors required for macropinocytosis [84,182,184,223]. Moreover, it is known that macropinocytosis is active in macrophages [224] and DCs [225], primary targets of EBOV. After uptake, virions are trafficked to late endosomes where GP is primed by the cysteine proteases CatB and L.

Cathepsin $B$ and $L$. Cathepsins are divided into three families according to the amino acid present in their active site: serine, aspartate and cysteine cathepsins. The latter are members of the family of papain-like cysteine proteases. At sequence level, there are 11 human cysteine cathepsins, B, C, F, H, K, L, O, S, V, X, and W [226-228]. Cathepsins are synthetized as preproenzymes. The signal peptide is cleaved by signal peptidase during the import of the nascent polypeptide chain into the ER. The propeptide maintains cathepsins in their inactive form while they are trafficked through the ER and Golgi apparatus. Once sorted into late endosomes they are proteolytically processed into their mature and proteolytically active form [229-232]. Most cathepsins have endopeptidase activity. However, CatB can act as carboxypeptidase [233,234]. They require a slightly acidic environment, such as the one found in endosomes and lysosomes, to have proper proteolytic activity $[230,235]$. Cathepsins are ubiquitously and redundantly expressed. Hence, cathepsins can be upregulated to take over the function of a related enzyme that has been eliminated by gene knockout or inhibited $[227,229,236-238]$.

CatB and CatL are involved in antigen processing and presentation, and apoptosis $[239,240]$. Also, they have been identified as the proteases responsible for filovirus GP priming in endosomes [220,241-243]. Priming of EBOV-GP by CatB/L occurs in two steps: First, GP1 (130 kDa) is cleaved by CatB and/or CatL into a $50 \mathrm{kDa}$ form, that is 
further cleaved, again by CatB/L or probably by other host proteases into a $19 \mathrm{kDa}$ form [242,244-246]. In this step, the glycan cap and the MLD are removed by these enzymes, exposing the RBD and potentiating the capacity for membrane fusion. The GP1-RBD remains associated with GP2 via a disulfide bond in a metastable conformation $[82,244,245]$. Schornberg and colleagues showed that virions bearing the $19 \mathrm{kDa}$ GP1 (achieved by processing virions bearing EBOV-GP with thermolysin, a bacterial derived protease that mimics $\mathrm{CatB} / \mathrm{L}$ cleavage, and recombinant $\mathrm{CatB} / \mathrm{L}$ ) depicted higher infectivity as compared to untreated virions which harbor unprocessed GP. Furthermore, it was demonstrated that entry of virions containing the $19 \mathrm{kDa}$ GP1 was largely resistant to $\mathrm{CatB} / \mathrm{CatL}$ inhibitors but remained sensitive to a lysosomotropic agent and a cysteine protease inhibitor [87,242,243], suggesting that GP is first cleaved by CatB/CatL and subsequently another lysosomal factor, likely a thiol reductase, is required to trigger membrane fusion. It is important to note that CatB/L dependence varies among filoviruses, for instance CatB activity is required for EBOV-, TAFV- and BDBV- but not for SUDV-, RESTV- or MARV-GP-driven entry [247]. Moreover, several but not all studies demonstrating a role of CatB/L in filovirus entry used vesicular stomatitis virus (VSV) [248] or murine leukemia virus (MLV) [249] vectors pseudotyped with filovirus GPs [247,250] and shape and size of these particles differs significantly from those of authentic filoviruses. Notably, Marzi and colleagues showed that $\mathrm{CatB}^{-/}$or $\mathrm{Cath}^{-/}$knockout mice supported EBOV spread as efficient as wild type mice [251]. These contradictory findings suggest that protease choice of EBOV in cell culture might not adequately reflect protease choice in the infected host or that EBOV can use proteases other than CatB/L to secure GP priming in the host.

\subsubsection{Receptor binding}

After GP priming by CatB/L, the primed EBOV-GP interacts with the intracellular receptor NPC1 $[85,86]$, which, jointly with an unknown stimulus, triggers membrane fusion, the final step of viral entry.

Niemann-Pick C1. NPC1 is a late endosomal/lysosomal protein that is ubiquitously expressed. It has 13 transmembrane-spanning helices (sterol sensing domain, SSD), and 3 luminal domains, domain A ( $\mathrm{N}$-terminal domain, NTD), domain $\mathrm{C}$ and domain I [252-255]. NPC1 has been implicated in cholesterol homeostasis and absorption. To 
accomplish this function, NPC1 (NTD domain and domain C loop 2) binds to Niemann-Pick C2 (NPC2) which in turn binds to cholesterol [256-259]. Abrogation of NPC1 or NPC2 function leads to cholesterol accumulation in lysosomes, causing the fatal Niemann-Pick disease [252,260-262]. NPC1 was first identified to be an important entry factor for EBOV by two studies: the first one, performed by Carette and colleagues, used genome-wide screening of haploid human cells to identify those that were not compatible with filovirus GP-driven entry [86]. The second study was done by Côté and coworkers, who screened a library of chemical compounds for filovirus entry inhibitors, including U18666A, which mimics Niemann-Pick disease [85]. Later it was shown that U18666A blocks EBOV-driven entry into host cells, despite the fact that it binds to a different domain (SSD) than EBOV-GP (domain C). However, the concentration of U18666A needed to block EBOV-driven entry is 100-fold higher $[85,87,263,264]$ than the one needed to inhibit cholesterol trafficking. It was hypothesized that when applied at high concentrations, U18666A and other cationic amphiphiles may interact with the NPC1 C-loop 2 with low affinity but sufficient to block virus entry [265]. Further, studies revealed that primed EBOV-GP but not unprocessed EBOV-GP binds to loops 1 and 2 from domain C, indicating that the RBD has to be fully exposed in order to interact with NPC1 $[255,266,267]$. The in vivo relevance of NPC1 was demonstrated by Herbert and colleagues, using NPC1 1 mice [268]. They could show that these mice were completely resistant to mouse-adapted EBOV infection, which indicates that NPC1 expression is essential for filovirus replication and pathogenesis, making it a promising therapeutic target. In sum, NPC1 is an intracellular receptor of EBOV and interactions of primed GP with NPC1 in conjunction with low pH might help release the internal fusion loop (IFL) and facilitate the conformational changes in GP required for membrane fusion $[93,255,269]$. 


\subsection{Ebola virus and the interferon system}

\subsubsection{Innate immune system}

All live forms are constantly exposed to pathogens, and humans are no exception. However, the immune system allows us to prevent and or control infections. In vertebrates, the immune system is divided in an innate and an adaptive arm. Adaptive responses are highly specific but slow, taking between one or two weeks to develop upon the first exposure to a pathogen, since specific clones of $B$ and $T$ cells reactive against the new pathogen need to be activated and proliferate [270]. Subsequently, the adaptive but not the innate responses lead to the development of immunologic memory. Thus, memory $B$ and $T$ cells have the ability to respond more rapidly to subsequent encounters with the same antigen or pathogen and may provide lifelong protection [271].

The innate immune system is not only present in vertebrates, but also in invertebrates and plants. It is the first line of defense against pathogens, taking only minutes to activate and hours to remove the threat. It plays a critical role in activation and regulation of the adaptive immune system [272,273]. The innate immune system is known to be a non-specific response against pathogens. However, this concept is changing. New evidence suggests that NK cells and macrophages can develop specific immune memory based on recognition of certain PAMPs. After PAMP recognition, epigenetic changes at the level of histone methylation and acetylation reprogram the cells, thereby increasing their activity upon secondary stimulation with the same or similar stimuli and providing protection in a T/B-cell-independent manner [274-276]. The main components of the innate immunity include physical barriers (i.e. skin, mucosal membranes, and gut-associated lymphoid tissue), chemical barriers (i.e. low $\mathrm{pH}$, hydrolytic enzymes, antimicrobial peptides and pro-inflammatory cytokines) present on the surface of physical barriers. The third component includes a cellular response, carried out by phagocytic cells (macrophages, neutrophils, monocytes and DCs), which is triggered upon invasion by a pathogen that was able to overcome the epithelial barriers [272,277].

Macrophages, neutrophils and DCs are able to recognize pathogen-associated molecular patterns (PAMPs), including lipopolysaccharide (LPS), peptidoglycans or 
complex carbohydrates present on bacteria, fungi and other pathogens. PAMPs are sensed by PRRs and PRR binding triggers gene expression of proteins of the innate immune system (e.g. interferons, defensins, cytokines) [272,278,279].

\subsubsection{Interferon system}

The interferon (IFN) system is an important innate defense against viral infections. IFNs are divided in type I, type II and type III, based on their structural homology, chromosomal location and interaction with their receptor chains. Type I IFNs include IFN- $\alpha$, IFN- $\beta$, IFN- $\varepsilon$, IFN- - , and IFN- $\omega$ and are essential for eliminating viral infections. Type II IFNs (IFN-y) are also required for defense against viral infection. In addition, they regulate expression of major histocompatibility complex and modulate the adaptive immune response [272,277,280,281]. Type III IFNs comprise IFN- $\lambda 1$, IFN- $\lambda 2$, IFN- $\lambda 3$ and IFN- $\lambda 4$. They display similar functions as type I IFNs [282-284].

Expression of IFNs is induced in infected cells upon recognition of PAMPs by PRRs such as toll-like receptors (TLRs), retinoic acid-inducible gene-I (RIG-I)-like receptors (RLRs), and nucleotide-binding oligomerization domain (NOD)-like receptors (NLRs) [285]. TLRs are membrane spanning proteins with a leucine-rich repeats (LRRs) extracellular region and toll-IL-1 receptor (TIR)-domains. So far, 10 TLRs have been identified in humans. They can be expressed in the plasma membrane (TLR1, TLR2, TLR4, TLR5, TLR6) or in endosomes/lysosomes (TLR3, TLR7, TLR8, TLR9). The TLRs responsible for sensing viral PAMPs are: TLR3 (double stranded RNA, dsRNA), TLR7 and TLR8 (single stranded RNA, ssRNA), and TLR9 (unmethylated CpG) $[272,286]$.

RLRs and NLRs receptors are cytosolic PRRs. RLRs are RNA helicases with caspase recruitment domains (CARD) that recognize different species of RNAs: RIG-I binds to short dsRNA and 5'-tri- or diphosphate ssRNA [287-289]. MDA5 recognizes long dsRNA and viral mRNAs lacking 2'-O-methylation [290,291]. The third RLR is LGP2, which can enhance MDA5 activation when binding to dsRNA in tandem with MDA5, and inhibit RIG-I by direct interaction or by competing for dsRNA [292-294]. NLRs induce production of immune and inflammatory responses. They are able to recognize flagellin, LPS, peptidoglycans, bacterial toxins, and nucleic acids from bacteria and 
viruses. The best characterized NLRs are NOD1 and NOD2, similar to RLRs, they have a CARD domain and can recognize breakdown products formed during synthesis or degradation of the bacterial cell wall. NOD2 can also activate responses against viruses like IAV [272,295,296].

After viral PAMPs recognition, a signaling cascade to produce nuclear factor kappa-light-chain-enhancer of activated B cells (NF-KB), IFN-regulatory factors (IRFs) and type I and III IFNs is triggered. First, PRRs interact with their adapter proteins (TLRs: TRIF, MyD88; RLRs: MAVS). In the case of NLRs the cascade starts with NOD binding to the serine/threonine kinase RIP2. Adaptor proteins then recruit TRAF ubiquitin ligases, IKKa/ $\beta$ and TBK1 kinases. Subsequently, phosphorylation, dimerization, activation and nuclear translocation of IRF3, IRF7, and NF-KB takes place. Finally, these protein bind to the promoter region of IFN-stimulated elements inducing expression of type I and III IFNs and some IFN-stimulated genes (ISGs) [272,297-302]. IFNs are secreted into the extracellular space, where they bind to IFN receptors (IFNR1, IFNR2, IFNLR) on neighboring cells [303]. IFN receptor binding leads to the activation of the JAK/STAT pathway. In short, Janus-kinases (JAK-1 and TYK2) are activated upon IFN binding and phosphorylate signal transducer and activator of transcription (STAT) factors 1 and 2. This leads to the formation of the IFN-stimulated gene factor 3 complex via the interaction of STAT1 and STAT2 with IRF9. Finally, this complex translocates into the nucleus, binds to IFN-stimulated response elements and triggers expression of over 500 ISGs [300,301,304-306].

\subsubsection{Interferon induced genes}

IFNs induce a so-called antiviral state, and synthesis of different ISGs helps to inhibit different stages of the viral replication cycle. In the following paragraphs some of the most characterized will be discussed.

Myxovirus resistance $(M x)$ proteins. Mx proteins belong to the family of dynamin-like large guanosine triphosphatases (GTPases). They have an N-terminal GTPase (G) domain, a middle domain (MD), and a C-terminal GTPase effector domain (GED). The $M D$ and GED domains are important for Mx antiviral activity, self-oligomerization and formation of the ring-like structures. These ring-like structures bind to the viral 
nucleocapsid, induce conformational changes and stimulate GTPase activity. In human cells two Mx proteins are expressed, Mx1 (MxA) and Mx2 (MxB) [307-310]. They inhibit early stages of the virus replication cycle and display broad antiviral activity and. For example, MxA traps incoming viral nucleocapsids from IAV or Thogoto viruses, preventing their import into the nucleus. Also, it can inhibit mRNA synthesis of VSV and human parainfluenza viruses [311-314]. Human MxB prevents integration of HIV-1 into the host genome by targeting the viral capsid and inhibiting uncoating, nuclear uptake or integration by the viral pre-integration complex [315-318].

Human interferon-induced transmembrane (IFITM) proteins. IFITMs comprise four proteins: IFITM1, IFITM2, IFITM3 and IFITM5. IFITM1, 2, and 3 are viral restriction factors with constitutive and ubiquitous expression. However, upon IFN stimulation their expression is enhanced [319,320]. IFITM1 is mainly located at the plasma membrane while IFITM2 and IFITM3 localize in late endosomes, lysosomes and autolysosomes [321,322]. IFITM proteins inhibit the fusion of the viral envelope with the membrane of the target cell [323]. IFITMs inhibit viruses like IAV, coronaviruses, filoviruses, flaviviruses and HIV-1 [321,324,325]. There are two models that could explain IFITMs antiviral mechanism. One suggests that IFITM proteins alter endosome acidification, inhibit cellular proteases required for priming of viral glycoproteins [326,327] or redirect viruses to a non-fusogenic pathway [322,327]. The second mechanism proposes that IFITMs may inhibit membrane fusion by altering the biological properties of cellular membranes such as curvature and fluidity [328].

Tripartite motif (TRIM) family proteins. Tripartite motif (TRIM) family proteins are highly conserved proteins with a N-terminal RBBC motif, one or two B-box domains and a C-terminal coiled-coil domain. They are a group of E3 ubiquitin ligases involved in cell functions including cell cycle progression and autophagy, and can display antiviral activity [329,330]. TRIM5a is known to inhibit HIV-1 uncoating. Thus, recognition of incoming capsids by TRIM5 $\alpha$ accelerates uncoating and resulting in premature exposure of the nucleoprotein complex [331]. TRIM22 is another protein that inhibits HIV-1. TRIM22 targets Gag trafficking to the plasma membrane, resulting in decreased particle production [332]. In addition, TRIM22 can inhibit hepatitis B virus by preventing the transcription of viral genes [333], encephalomyocarditis virus by promoting 
ubiquitination of the viral C3 protease [334], and IAV by targeting the viral nucleoprotein for degradation [335].

2'-5'-oligo-adenylate synthetase (OAS) proteins and the protein kinase $R(P K R)$. Two examples of antiviral effector proteins that interfere with viral infection at the stage of protein synthesis are OAS proteins and PKR. The 2'-5'-OAS family in humans comprises four genes: OAS1, 2, 3 and OAS-like (OASL) and they are able to inhibit viral protein synthesis [336]. For this, they bind to dsRNA, synthesize 2',5'-linked phosphodiester bonds, which in turn form 2'-5'-olygoadenylates that activate the latent ribonuclease $L$ (RNase $L$ ). RNase $L$ then degrades cellular and viral RNAs [337,338]. Similar to OAS, PKR binds to viral dsRNA. PKR is constitutively expressed but its expression is upregulated upon IFN stimulation [339]. The antiviral activity of PKR relies on phosphorylation of the alpha unit of eukaryotic translation initiation factor 2 . Phosphorylation leads to sequestration of a guanine nucleotide exchange factor called elF2b which facilitates recycling of guanidine diphosphate (GDP) to guanidine triphosphate (GTP). This results in inhibition of both cellular and viral translation by preventing the conversion GDP to GTP [340,341].

Virus inhibitory protein, endoplasmic reticulum-associated, IFN-inducible (Viperin). ISGs can also target late steps of the replication cycle. One well-known example is viperin. Viperin is a virus inhibitory protein associated with the ER that can inhibit farnesyl diphosphate synthase. Decreased expression of this enzyme interferes with cell membrane fluidity. By altering fluidity of lipid rafts, it affects budding of enveloped viruses that require these microdomains, such as IAV and HIV-1 [342-344]. Furthermore, it has been shown that viperin inhibits RNA replication of HCV by binding to the non-structural 5A protein and interfering with the stability and function of the viral replication complex [345]. Another ISG that inhibits a late step in viral replication is tetherin and this protein will be discussed in detail in the following chapter. 


\subsubsection{Tetherin}

Tetherin, also known as BST-2, HM1.24 or CD317, is constitutively expressed and its expression is upregulated by type I and II IFNs [346,347]. It was first identified as a surface marker of terminally differentiated normal and neoplastic B cells $[348,349]$. Tetherin is a type II transmembrane protein composed of 180 amino acids and exhibits the following domain organization: A N-terminal cytoplasmic tail, a transmembrane domain (TMD), followed by a coiled-coil ectodomain and a C-terminal glycosylphosphatidylinositol (GPI)-anchor [350,351]. Tetherin forms homodimers via disulfide bonds between cysteine residues 53,63 , and 91 [352,353], which are located in the extracellular domain. It is mainly located at the plasma membrane within lipid rafts but it can also be found in the trans-Golgi network (TGN) and in vesicular compartments $[350,354]$. The GPI anchor of tetherin is essential for its transport through the ER and localization in lipid rafts, while the cytoplasmic tail, via a conserved double tyrosine motif, mediates endocytic recycling through the TGN in a clathrin-dependent manner $[355,356]$. Also, upon virus tethering the cytoplasmic tail of tetherin can induce activation of NF-KB, resulting in the expression of ISGs and thereby amplification of the innate immune response $[357,358]$.

The unique structure and domain organization of tetherin, having two membrane anchors, allows it to inhibit release of budding virions from infected cells by forming a physical tether between them. This ability was discovered when HIV-1 virions, lacking viral protein $U(\mathrm{Vpu})$ were retained at the cell surface [359-361]. Later it was shown that tetherin prevents release of several enveloped virus families such as rhabdo- [362], alpha- [363,364], arena-, paramyxo-, orthomyxo- [365,366], herpes- [367], orthohepadna- [368], flavi- [369-371], and filoviruses [372,373].

There were three proposed mechanism by which tetherin cross-links virions. First, one molecule within a tetherin dimer might insert into envelope of the budding virus, while the second molecule remains on the host cell membrane. In this scenario, the disulfide bonds between the ectodomains of the tetherin molecules are responsible for the tether effect [374]. A study performed by Fitzpatrick and colleagues provided evidence that supports this theory. Treatment with phosphatidyl inositol-specific phospholipase $\mathrm{C}$, an enzyme that cleaves the GPI anchor, did not efficiently release tethered virions 
from the cell surface [375]. The second mechanism suggests that both GPI-anchors of a tetherin homodimer are taken up by the virus, in a parallel orientation, and the TMDs remain inserted into the plasma membrane, or vice versa [375]. Findings that support this scenario are that dithiothreitol treatment, which reduces disulfide bonds, failed to release tethered particles [375]. Furthermore, data observed via cryo-electron tomography showed that expected and experimental distance between virions and cell membrane matched, when the coiled-coil domains of tetherin are extended, [376]. Finally, an antiparallel orientation to tether virions has been suggested. In this configuration, the GPI anchor of one tetherin molecule and the TMD of the second one are incorporated into the virion while their opposite membrane anchors remain inserted in the plasma membrane [376,377]. A study performed by Venkatesh and Bieniaz provided conclusive proof that the tetherin conformation associated with antiviral activity are homodimers in which tetherin monomers are assembled in a parallel fashion and in which the GPI anchors are preferably inserted into the viral membrane [378]. Studies to identify determinants important for tetherin antiviral activity concluded that domain organization, topology, and intracellular transport are more important than amino acid sequence. Most importantly, Perez-Caballero and colleagues found that a synthetic molecule that was engineered to have the same domain organization as tetherin but not sequence homology was able to restrict viral release [360].

The in vivo relevance of tetherin has been shown in tetherin-deficient mice. In Chikungunya infected mice, viral load at the inoculation site was higher in tetherin deficient animals than in wild type mice and more prominent infection of lymphoid tissue was observed in the absence of tetherin [379]. Similar results were found when tetherin knockout mice were infected with murine retroviruses including mouse mammary tumor virus and MLV $[380,381]$. Furthermore, studies conducted with Friend retrovirus showed that wild type mice had increased $\mathrm{CD} 4^{+}$and $\mathrm{CD} 8^{+} \mathrm{T}$ cell responses as compared to tetherin knockout mice. These responses were critical for recovery and are possibly driven by tetherin cytoplasmic tail signaling [382]. Thus, tetherin can inhibit viral spread in the host and modulate immune response in vitro and in vivo. 


\subsubsection{Tetherin antagonists}

Several viruses susceptible to tetherin restriction evolved countermeasures - viral proteins that interfere with tetherin expression or antiviral activity at the site of viral budding or antiviral activity $([361,383]$ table 1$)$.

\begin{tabular}{|c|c|c|}
\hline Virus & Tetherin antagonist & Mechanism \\
\hline HIV-1 & Vpu $[359,384]$ & $\begin{array}{l}\text { Downregulation, degradation, } \\
\text { removal from lipid rafts }\end{array}$ \\
\hline HIV-2 & Env $[385,386]$ & Intracellular sequestration \\
\hline SIV & Nef/Env [387-389] & Downregulation \\
\hline KSHV & K5 [390] & Downregulation and degradation \\
\hline HSV & $\mathrm{gM}[391]$ & Downregulation \\
\hline SARS-CoV & ORF7a [392] & Glycosylation interference \\
\hline CHIKV & nSP1 [363] & Downregulation \\
\hline hPIV-2 & V protein [393] & Downregulation \\
\hline Sendaivirus & GP [394] & Unknown \\
\hline EBOV & GP $[372,373,395]$ & Unknown \\
\hline MARV & GP [365] & Unknown \\
\hline
\end{tabular}

Table 1. Tetherin antagonists and mechanisms.

The best studied tetherin antagonists is HIV-1 Vpu protein. Vpu's antagonism, relies on interaction between its TMD with the TMD of tetherin [396,397]. An AXXXAXXXAXXXW motif within Vpu's TMD was found to be crucial for tetherin-Vpu interaction, mutation of the alanine residues in this motif impairs the ability of $\mathrm{Vpu}$ to counteract tetherin $[396,398]$. As mentioned above, the mechanisms employed by Vpu to counteract tetherin include cellular surface down-regulation and degradation of tetherin [384,399-401]. For degradation, tetherin is ubiquitinated and targeted for lysosomal degradation in the presence of Vpu [401,402]. For this, Vpu recruits E3 ubiquitin ligase via a conserved phosphoserine motif in its cytoplasmic tail. Mutation of this motif prevents Vpu's interaction with the ubiquitin ligase complex, B-TrCP2, and hence the initiation of the ubiquitination cascade and ultimately tetherin antagonism [403,404]. Furthermore, Vpu inhibits the anterograde transport of tetherin by preventing proper cellular transport of newly synthetized tetherin from the ER and/or 
from early endosomes to the cell surface [397,405]. In the case of tetherin removal from lipid rafts, Vpu forms a complex with tetherin via the tryptophan residue 76 of the cytoplasmic tail of $\mathrm{Vpu}[401,406]$. This anchors the C-terminus of Vpu to the lipid bilayer and displaces tetherin from budding sites [406]. However, tetherin antagonism by several viral proteins other than Vpu has in not been fully elucidated and it is largely unclear how tetherin is antagonized by EBOV-GP.

\subsubsection{EBOV-GP as a tetherin antagonist}

Kaletsky and colleagues demonstrated that EBOV-GP like HIV-1 Vpu can promote release of VP40-based, EBOV-like particles from tetherin-positive cells [395]. Moreover, they were able to prove that EBOV-GP interacts with tetherin, a finding confirmed by separate studies, without altering tetherin expression levels. Additionally, they provided initial insight on the domains within GP involved in tetherin antagonism. Thus, they demonstrated that SGP and shed GP, which lack the TMD and cytoplasmic tail of EBOV-GP, did not inhibit tetherin, indicating that full-length GP is required for tetherin antagonism. However, deletion of the MLD did not affect tetherin counteraction by GP. Later, Lopez and colleagues and other laboratories showed that Vpu requires a specific sequence within the tetherin TMD to counteract tetherin and target it for degradation while EBOV-GP was able to antagonize even artificial tetherin and did not remove tetherin from the cell surface [372]. Radoshitzky and colleagues confirmed previous findings showing that release of virus-like partices (VLPS) based on the matrix proteins of several enveloped viruses, including EBOV, HIV-1 and Lassa virus (LASV) are restricted by tetherin and that co-expression of several viral antagonist could rescue viral release. In contrast, EBOV-GP and MARV-GP failed to rescue LASV-like particles release from tetherin-positive cells [365] and the reasons for the failure of GP to promote particle release from tetherin-positive cells is at present unknown. Finally, they demonstrated that tetherin expression does not reduce EBOV infection [365], a finding confirmed by another study [407], and that GP and tetherin fail to colocalize in infected cells. Whether these findings indicate that GP-mediated tetherin antagonism promotes viral spread in tetherin-positive cells or that EBOV-release is intrinsically not sensitive to tetherin remains to be elucidated. 
Kühl et. al. and subsequent studies showed that EBOV-GP can antagonize tetherin orthologues of diverse species [360,373,408], confirming that tetherin antagonism is sequence independent. Furthermore, they provided evidence that EBOV-GP interacts with tetherin via GP2 and confirmed that co-expression of GP does not modulate tetherin levels at the cell surface $[372,373]$. Lopez and colleagues investigated whether GP might remove tetherin from lipid rafts, which have been proposed as platform for EBOV budding $[103,409]$. However, they found that neither EBOV-GP nor Vpu affected localization of tetherin in lipid rafts and did not prevent colocalization of viral particles and tetherin at the budding sites. Furthermore, they did not find evidence of EBOV-GP being a lipid-raft protein, which contrasts previous findings [103].

Gustin and colleagues reported that EBOV-GP enhances egress of VP40-based VLPs by preventing the interaction of tetherin and VP40 $[395,410]$. Moreover, they found that GP can mask tetherin from recognition by antibodies and that tetherin is efficiently incorporated into VP40-based particles. A role for VP40 in tetherin sensitivity was also supported by a study conducted with MARV: It was reported that MARV-VP40 is largely resistant to inhibition by tetherin and that MARV adaptation to mice resulted in acquisition of mutations in VP40 that markedly increased sensitivity towards human but not mouse tetherin [411]. Collectively, these results suggest that both VP40 and GP can impact tetherin sensitivity and that GP might antagonize tetherin by disrupting VP40-tetherin interactions. Finally, it is noteworthy that tetherin antagonism by feline immunodeficiency virus (FIV) envelope (Env) glycoprotein was reported to entail Env-mediated exclusion of tetherin from virions or virion assembly sites and failed to promote release of HIV-based particles from tetherin-positive cells [412]. Unlike GP, FIV-Env can only counteract carnivore tetherins and only in the context of FIV particles. These findings suggest that Env affects tetherin sensitivity at the point of FIV budding and does not exert a cell-wide phenotype and the same might apply to EBOV-GP.

The studies discussed above demonstrated that the GP2 subunit interacts with tetherin [373] and a separate study showed that the TMD of EBOV-GP contributes to tetherin counteraction [413]. However, introducing the EBOV-TMD into LASV-GPC did not confer tetherin antagonism to LASV-GPC. This indicated that the TMD is not the only determinant in EBOV-GP that controls tetherin antagonism [413]. Indeed, during the 
course of the present thesis Vande Burgt and colleagues confirmed the importance of the TMD for tetherin antagonism [413,414] and showed that also the glycan cap of GP is important [414]. Moreover, Brinkmann and colleagues showed that mutations in the EBOV-GP RBD can abrogate tetherin antagonism [395,415] and that tetherin antagonism requires adequate GP glycosylation [415]. In sum, the TMD, glycan cap and RBD as well as proper glycosylation of EBOV-GP are required for GP driven tetherin antagonism but it is unknown how they contribute to tetherin antagonism. 


\section{Aims}

Ebola virus disease (EVD) is a severe disease that threatens human and animal health. Infection by Ebola virus (EBOV) is major cause of EVD and understanding how EBOV interacts with host cells and evades immune control might open novel avenues for antiviral intervention. The EBOV glycoprotein (GP) is the only viral surface protein and mediates the first step in EBOV infection, viral entry into target cells [82]. For this, EBOV-GP needs to be processed (primed) by the host cell proteases cathepsin B/L (CatB/L) [241], at least in cell culture. However, studies with $\mathrm{CatB}^{-/-}$or $\mathrm{CatL}^{-/-} \mathrm{knockout}^{-}$ mice indicated that these proteases are dispensable for viral spread in the infected host [251]. The reasons for the discrepancy between the in vitro and in vivo findings are poorly understood. Therefore, one goal of the present thesis was to identify determinants that control CatB/CatL dependence of EBOV entry.

Apart from mediating viral entry, EBOV-GP also counteracts the interferon-induced antiviral host cell factor tetherin $[82,395]$. It is conceivable that tetherin counteraction may allow EBOV to spread in tetherin-positive cells and tissues, and efficient spread of EBOV in tetherin transfected cells has been demonstrated. However, the determinants in GP that govern counteraction of tetherin and the contribution of this process to viral spread are incompletely understood [413-415]. Therefore, a second aim of this thesis was to identify determinants in EBOV-GP that contribute to tetherin antagonism and to obtain insights whether antagonism can promote viral spread in cell culture. A GXXXA motif within the transmembrane domain (TMD) of EBOV-GP was found to be required for the well-documented cytotoxic effects associated with expression of EBOV-GP in cell culture [416] and served as starting point for the thesis research. In summary, the aims of this thesis were to:

1) Identify determinants governing the CatB/L dependence of EBOV-GP-driven entry into host cells.

2) Identify the contribution of a GXXXA motif in EBOV-GP to tetherin antagonism and determine whether antagonism contributes to viral spread. 
4. Manuscripts

Calu-3 cells are largely resistant to entry driven by filovirus glycoproteins and the entry defect can be rescued by directed expression of DC-SIGN or cathepsin L.

Submitted to Virology, January 2019

A GXXXA Motif in the Transmembrane Domain of the Ebola Virus Glycoprotein Is Required for Tetherin Antagonism. Journal of Virology, 2018 


\subsection{First manuscript}

Calu-3 cells are largely resistant to entry driven by filovirus glycoproteins and the entry defect can be rescued by directed expression of DC-SIGN or cathepsin L.

González-Hernández, M., Hoffmann, M., Pöhlmann, S.

Virology, submitted on January $9^{\text {th }}, 2019$. Submission number: VIRO-19-3

Individual contributions:

I performed the experiments that resulted in the data shown in figure 1 (panels $A, D$, and $E$ ), figure 2 and figure 4 (panels $A-E$ ). Moreover, I analyzed all the data that I generated and I contributed to the writing of the manuscript. 
${ }^{\star}$ Manuscript

Click here to view linked References

1 Calu-3 cells are largely resistant to entry driven by filovirus glycoproteins and the

2 entry defect can be rescued by directed expression of DC-SIGN or cathepsin L

3

4 Mariana González-Hernández ${ }^{\mathrm{a}, \mathrm{b}}$, Markus Hoffmann ${ }^{\mathrm{a}}$ and Stefan Pöhlmann ${ }^{\mathrm{a}, \mathrm{b}}$

5

$6{ }^{\mathrm{a}}$ Infection Biology Unit, German Primate Center - Leibniz Institute for Primate Research,

7 Kellnerweg 4, 37077 Göttingen

$8{ }^{\mathrm{b}}$ Faculty of Biology and Psychology, Wilhelm-Weber-Str. 2, University Göttingen, 37073

9 Göttingen, Germany

10

11 Keywords: Filovirus, Ebola virus, Marburg virus, glycoprotein, host cell entry, cathepsin L, DC-

12 SIGN

13

14

15

16

17

18

19

20

21

22

23 
4

5

7

\section{ABSTRACT}

25 Priming of the viral glycoprotein (GP) by the cellular proteases cathepsin B and L (CatB, CatL) is

26 believed to be essential for cell entry of filoviruses. However, non-filamentous particles have

27 frequently been used to prove this concept. Here, we report that GP-mediated entry of spherical,

28 bullet-shaped and filamentous particles depends on CatB/CatL activity and that this effect is cell

29 line-independent. Moreover, we show that the human cell line Calu-3, which expresses low

30 amounts of CatL, is largely resistant to entry driven by diverse filovirus GPs. Finally, we

31 demonstrate that Calu-3 cell entry mediated by certain filovirus GPs can be rescued upon directed

32 expression of CatL or DC-SIGN. Our results identify Calu-3 cells as largely resistant to filovirus

33 GP-driven entry and demonstrate that entry is limited at the stage of virion attachment and GP 


\section{1. Introduction}

48 The family Filoviridae comprises three genera, Ebolavirus, Marburgvirus and Cuevavirus. To

49 date, there are five species of ebolaviruses, Zaire ebolavirus (only member: Ebola virus [EBOV])

50 Sudan ebolavirus (only member: Sudan virus [SUDV]), Bundibugyo ebolavirus (only member:

51 Bundibugyo virus [BDBV]), Tai Forest ebolavirus (only member: Tai Forest virus [TAFV]),

52 Reston ebolavirus (only member: Reston virus, [RESTV]), one species of marburgvirus,

53 Marburg marburgvirus (two members: Marburg virus [MARV] and Ravn virus [RAVV]) and

54 one species of cuevavirus, Lloviu cuevavirus (only member: Lloviu virus [LLOV]) (Kuhn, 2017).

55 There is compelling evidence that all members of the three genera circulate in bats, which are

56 believed to serve as their natural reservoir, and that zoonotic transmissions of most of these

57 viruses to humans can induce severe disease (Mandl et al., 2018; Olival and Hayman, 2014).

58 Multiple filovirus outbreaks in Africa were recorded during the last 52 years and usually entailed

59 less than 500 cases (Centers for Disease Control and Prevention, 2018). In contrast, an outbreak

60 of Ebola virus disease (EVD) in West Africa in 2013 resulted in an EVD epidemic with more

61 than 10.000 deaths and secondary transmission events in Europe and the US (Lo et al., 2017).

62 Further, a recent outbreak of EVD has started in the Democratic Republic of Congo, causing 543

63 confirmed cases and 309 deaths (WHO, 2018), and is still ongoing (as of January 2019). These

64 examples show that filovirus infection constitutes a serious health threat that is not limited to

65 African countries in which most filoviruses are endemic. Despite promising results of clinical

66 trials, neither vaccines nor antiviral agents approved by regulatory agencies are currently

67 available to combat filovirus infection. Therefore, the development of antiviral strategies remains

68 a research focus and host cell factors required by diverse filoviruses for spread but dispensable

69 for host cell survival are potential drug targets. 
71 O-glycosylated type I transmembrane protein and it is the sole viral protein incorporated into the 72 viral envelope (Martin et al., 2016). Host cell entry of filoviruses is best studied for EBOV and 73 we will therefore focus the subsequent discussion on factors important for EBOV GP-driven 74 entry. To initiate infection, EBOV particles first attach to target cells, which can be facilitated by 75 GP interactions with attachment factors like the C-type lectin DC-SIGN (Alvarez et al., 2002; 76 Simmons et al., 2003). In addition or alternatively, attachment can be facilitated in a GP77 independent fashion: Phosphatidylserine residues on the viral envelope can be recognized by 78 TIM (T-Cell Immunoglobulin And Mucin Domain-Containing) proteins (Jemielity et al., 2013; 79 Kondratowicz et al., 2011; Moller-Tank et al., 2013) or, via an adaptor, by tyro 3 kinases, 80 including Axl, which are located on the cell surface (Shimojima et al., 2006). After attachment, 81 virions are taken up into cells by macropinocytosis (Quinn et al., 2009; Saeed et al., 2010) and 82 are trafficked into late endosomes, where GP is processed by the host cell cysteine proteases 83 cathepsin B and L (CatB, CatL) (Chandran et al., 2005; Kaletsky et al., 2007; Schornberg et al., 84 2006), which are known to also process several other viral glycoproteins (Pager et al., 2006;

85 Simmons et al., 2005). Proteolytically processed GP (also termed primed GP) then interacts with 86 the cholesterol transporter Niemann-Pick C1 (NPC1) (Carette et al., 2011; Cote et al., 2011) and, 87 upon low pH exposure and a poorly defined additional stimulus (Bale et al., 2011; Brecher et al., 88 2012; Schornberg et al., 2006) mediates the fusion of viral and late endosomal membranes, 89 thereby allowing the release of the viral genetic information in the host cell cytoplasm. Priming 90 of GP by CatB and to a lesser degree CatL is essential for host cell entry and results frequently 91 obtained with surrogate systems indicate that entry driven by the GPs of all filoviruses might 92 depend on CatB/CatL activity (Gnirss et al., 2012; Maruyama et al., 2014; Misasi et al., 2012). 
93 However, the relative dependence varies, with RESTV showing generally modest CatB/CatL

94 dependence and MARV exhibiting pronounced CatL (Misasi et al., 2012) but not CatB

95 dependence (Gnirss et al., 2012; Misasi et al., 2012). Moreover, EBOV entry into dendritic cells

96 was reported to be CatB but not CatL dependent (Martinez et al., 2010). Notably, a study by

97 Marzi and colleagues conducted with authentic viruses suggested that EBOV and BDBV entry

98 into cultured cells is CatB dependent while entry of several other ebolaviruses is not (Marzi et al.,

99 2012). Moreover, the study showed that lack of CatB or CatL expression is compatible with full

100 viral spread and pathogenesis in the rodent host (Marzi et al., 2012). The discrepancies between

101 these results and the CatB/CatL dependence reported by several other studies are incompletely

102 understood. However, one can speculate that particle shape - filoviruses are filamentous while

103 bullet-shaped or spherical particles are frequently used to model filovirus entry - and target cell

104 type may play a role.

105 Here, we show that EBOV-GP-driven entry is CatB/CatL dependent irrespective of the 106 surrogate system, particle shape and target cell type. Moreover, we demonstrate that Calu-3 cells

107 express very low levels of endogenous CatL, in keeping with a previous study (Park et al., 2016),

108 and are largely refractory to entry driven by ebola-, marburg- and cuevavirus GPs. Finally, we

109 show that entry into Calu-3 cells can be rescued by directed expression of DC-SIGN or CatL,

110 suggesting that attachment and GP priming can limit entry into these cells. 


\section{Results}

\subsection{EBOV-GP-driven entry into cell lines requires CatB/CatL activity irrespective of the shape}

of the GP-bearing particle and the target cell type

We first examined whether retroviral (spherical), rhabdoviral (bullet-shaped) and filoviral (filamentous) particles differ in their CatB/CatL-dependence for host cell entry. For this, we used retro- and rhabdoviral vectors previously used to analyze EBOV-GP-mediated host cell entry (Takada et al., 1997; Wool-Lewis and Bates, 1998) as well as replication competent vesicular stomatitis virus (VSV) encoding EBOV-GP (Gonzalez-Hernandez et al., 2018). Moreover, we employed two systems that depend exclusively on filovirus proteins. First, we produced EBOVlike particles harboring luciferase by directed expression of all viral proteins from plasmids, including a VP30 version that is fused to firefly luciferase (Dietzel et al., 2017). Second, we employed the EBOV trVLP system in which a truncated EBOV minigenome is packaged into filoviral particles due to expression of the missing open reading frames (EBOV-NP, -VP30, VP35 and - L) in trans (Watt et al., 2014). While the VP30-Luc system allows measuring a single delivery of VP30-luciferase into cells, the trVLP system allows studying entry and spread of filovirus-like particles. Finally, we employed the endosomal cysteine protease inhibitors E-64d and MDL28170 to block CatB/CatL activity in target cells. These inhibitors were previously shown to interfere with CatB/CatL activity (Brana et al., 1999; Gewies and Grimm, 2003; Hoffmann et al., 2016; Simmons et al., 2005) and were previously used to inhibit EBOV-GPdriven entry (Gnirss et al., 2012; Kaletsky et al., 2007). 
Analysis of GP-driven entry into 293 T cells revealed that entry was blocked by E-64d and MDL28170 in a concentration-dependent manner, irrespective of whether GP was presented on retro-, rhabdo- or filovirus particles (Fig. 1). In contrast, entry driven by the glycoprotein (G) of vesicular stomatitis virus (VSV) was not affected by E-64d and MDL28170, as expected.

Moreover, analysis of entry of EBOV-GP-bearing rhabdoviral particles into six different cell lines of human (accidental host; 293T, Huh-7, A549 and Calu-3), non-human primate (accidental host; Vero E6) and fruit bat (suspected reservoir host; EpoNi/22.1) origin revealed that entry was sensitive to inhibition by E-64d irrespective of the target cell type, although it should be stated that entry into Calu-3 was very close to background (Fig. 2). Collectively, our results show that, under the experimental conditions chosen, EBOV-GP-driven entry was CatB/CatL-dependent irrespective of the type of GP-bearing particle and target cell line. Moreover, our results identify Calu-3 cells as one of the very few cell lines that are largely resistant to GP-mediated entry.

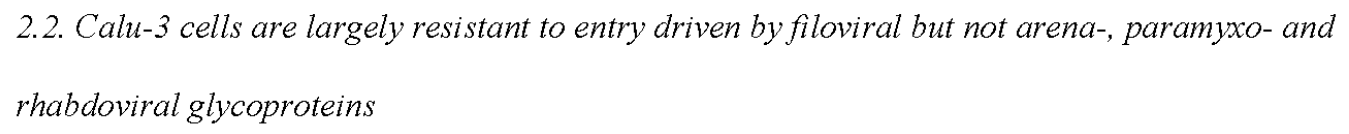
phenotype was specific for EBOV-GP or could also be observed for other viral glycoproteins. In order to answer this question, we investigated whether glycoproteins from viruses of diverse viral families also fail to mediate efficient Calu-3 entry or whether this effect is rather specific for EBOV-GP. We found that glycoproteins of arena- (Lassa virus [LASV], lymphocytic choriomeningitis virus [LCMV] and Machupo virus [MACV]), paramyxo- (Mumps virus [MuV] and Nipah virus [NiV]) and rhabdoviruses (VSV) mediated entry into Calu-3 cells with high 
efficiency and to roughly the same levels as measured for Vero E6 cells (Fig. 3A). EBOV-GPdriven entry into Vero E6 cells was highly efficient and comparable to that measured for the other glycoproteins tested, while entry into Calu-3 cells was severely attenuated ( 200 -fold compared to Vero E6 cells) (Fig. 3A). In order to investigate whether Calu-3 cells are largely refractory to entry driven by filovirus GPs in general, we performed the same experiment but tested GPs from viruses of all known filovirus species. All GPs tested mediated efficient entry into control Vero E6 cells, with entry driven by LLOV-GP being slightly less efficient than that observed for the other GPs (Fig. 3B). In contrast, Calu-3 cell entry was severely attenuated for all filovirus GPs with MARV-GP-driven entry being most efficient and ranging $\sim 10$-fold over background (Fig. 3B). These results reveal that Calu-3 cells are poorly susceptible to entry driven by filoviral glycoproteins, with the exception of MARV-GP, but are readily susceptible to entry driven by arena-, paramyxo- and rhabdoviral glycoproteins.

2.3. Directed expression of DC-SIGN or CatL can rescue Calu-3 cells entry driven by several but not all filovirus GPS

A previous study found that Calu-3 cells express very low amounts of endogenous CatL (Park et al., 2016). Quantitative RT-PCR analysis confirmed the presence of low amounts of CatL transcripts as compared to 293 T ( $\sim 183$-fold higher levels than Calu-3) and Huh-7 cells $(\sim 264-$ fold higher levels than Calu-3) (Fig. 4A). In contrast, CatB transcript numbers in Calu-3 cells were comparable or only slightly lower than those in $293 \mathrm{~T}$ and Huh7 cells ( $\sim 3-$ and 13-fold), respectively (Fig. 4A). Therefore, we next asked whether the low endogenous CatL levels in Calu-3 cells were responsible for inefficient GP-mediated cell entry. In parallel, we examined 
whether directed expression of other cellular factors known to promote EBOV entry could rescue entry efficiency. To address these questions, we transduced Calu-3 cells with vectors encoding CatB, CatL, Axl, DC-SIGN and NPC1, selected transduced cells via antibiotics and then examined protein expression by immunoblot. These analyses revealed robust expression of CatB, CatL, DC-SIGN, NPC1 and Axl, indicating that transduction and selection were successful (Fig. 4B).

Next, we determined whether the directed expression of the entry factors stated above modulated entry driven by EBOV-GP and other filovirus GPs. All particles examined were readily able to transduce Vero E6 control cells with high efficiency, whereas entry into Calu-3 cells was poor (Figs 3B and 4C). In comparison, directed expression of Cat $L$ in Calu-3 cells strongly increased transduction driven by MARV-GP ( $\sim 51.6$-fold) (Fig. $4 \mathrm{C})$. In addition, a moderate increase was observed for EBOV-GP-, SUDV-GP-, TAFV-GP- and RESTV-GP-driven entry ( 2.4-, $\sim 3.3-, \sim 2.5$ - and $\sim 5.6$-fold, respectively), while no appreciable enhancement was observed for entry driven by BDBV-GP and LLOV-GP ( 1.3-fold, both). Moreover, directed expression of DC-SIGN in Calu-3 cells robustly promoted entry driven by EBOV-GP $(\sim 15.9-$ fold), SUDV-GP ( 24.1-fold), and MARV-GP ( 81.9-fold). For particles bearing RESTV-GP or TAFV-GP entry efficiency was moderately increased ( $\sim 3.7$ - and $\sim 2.4$-fold, respectively), whereas entry driven by BDBV-GP and LLOV-GP was again almost at background levels ( $\sim 1.5$ - and $\sim 1.8$-fold increase, respectively) (Fig. 4C). Finally, expression of the other entry factors did not modulate transduction mediated by any of the filovirus GPs and none of the factors had an impact on transduction mediated by VSV-G, as expected (Fig. 4C). These results indicate that filovirus GP-driven entry into Calu- 3 cells is limited at the stage of attachment and priming and that the severity of these limitations varies among the GPs of different filovirus species. 
2.4 Augmentation of filovirus GP-driven entry into Calu-3 cells via directed expression of CatL or DC-SIGN can be blocked by a cathepsin L inhibitor and a DC-SIGN antibody, respectively

We next sought to provide formal proof that the increased filovirus GP-driven entry into Calu-3 cells expressing DC-SIGN and CatL as compared to Calu-3 control cells was a direct consequence of the expression of these entry factors. For this, we investigated whether entry augmentation could be blocked by the CatL inhibitor MDL28170 and an antibody directed against DC-SIGN. We selected particles bearing MARV-GP for these experiments, since MARV-GP-driven entry into CatL and DC-SIGN expressing cells was markedly more efficient than entry into Calu-3 control cells. Directed CatL expression in Calu-3 cells increased the efficiency of MARV-GP-driven entry as expected and pre-treatment with MDL28170 abolished this effect (Fig. 4D). Similarly, directed expression of DC-SIGN augmented entry, confirming our results discussed above, and augmentation was partially abrogated by pretreatment of target cells with a monoclonal antibody directed against the ectodomain of DC-SIGN (Fig. 4E). In contrast, entry of control particles bearing VSV-G was not sensitive to DC-SIGN antibody or MDL28170. These results indicate that directed expression of CatL or DC-SIGN augments MARV-GP-driven Calu-3 cell entry through direct effects. 


\section{3. Discussion}

231 Controversial results regarding the requirement of CatB/CatL activity for filovirus entry have 232 been reported (Chandran et al., 2005; Marzi et al., 2012) and differences in the experimental 233 systems used to study GP-driven entry might account, at least in part, for the discrepant 234 observations. Our study shows that EBOV-GP-driven entry into different cell lines depends on $235 \mathrm{CatB} / \mathrm{CatL}$ activity and that $\mathrm{CatB} / \mathrm{CatL}$ dependence is irrespective of the shape of the GP 236 presenting particles. Moreover, we identify Calu-3 cells as largely resistant to entry driven 237 EBOV-GP and other filovirus GPs and found that entry can be restored by promoting attachment 238 and/or CatL-dependent GP priming. that relied on both surrogate systems and infectious EBOV (Chandran et al., 2005). Moreover, subsequent analyses performed with surrogate models and/or authentic filoviruses confirmed CatB/CatL dependence of EBOV-GP-driven entry but also provided evidence that $\mathrm{CatB} / \mathrm{CatL}$ dependence of entry driven by other filoviral GPs was variable (Chandran et al., 2005; Gnirss et al., 2012; Martinez et al., 2010; Misasi et al., 2012; Sanchez, 2007). For instance, directed expression of CatB in CatB/CatL double knockout cells increased EBOV-GP- and TAFV-GPdriven entry while directed expression of CatL had only a moderate effect. Moreover, expression of both proteases only moderately increased SUDV-GP-driven entry. In contrast, directed expression of either CatB or CatL markedly increased RESTV-GP-driven entry and expression of CatL but not CatB efficiently promoted MARV-GP-driven entry (Misasi et al., 2012). The general requirement for CatB and/or CatL activity for entry driven by most ebola- and marburgvirus glycoproteins demonstrated by these studies was contrasted by a report by Marzi and colleagues, who showed that Vero cell infection by EBOV and to some degree BDBV 
253 depends on CatB but not CatL activity while entry driven by TAFV, SUDV and RESTV is 254 independent of CatB/CatL activities (Marzi et al., 2012). Finally, this study demonstrated that 255 knockout of CatB or CatL does not interfere with EBOV spread and pathogenesis in mice (Marzi 256 et al., 2012). The reason for the discrepancy between these results is largely unclear and accountable.

The present study shows that the shape of the particles used to study EBOV-GP-driven entry does not have a prominent impact on CatB/CatL dependence. Thus, 293T cell entry of spherical retroviral particles, bullet-shaped rhabdoviral particles and filoviral particles was inhibited by E-64d and MDL28170. In this context, it should be stated that particles produced using the trVLP system should adequately mimic virions produced in EBOV infected cells, since particle production in the trVLP system exclusively depends on viral proteins and all proteins encoded by EBOV are expressed in the particle producing cells (Watt et al., 2014). Importantly, the filamentous structure of these particles has been recently confirmed (Wang et al., 2018), thereby supporting our overall conclusion that particle shape is not a major determinant of CatB/CatL dependence of EBOV-GP-driven entry.

Analysis of lung- (A549, Calu-3), liver- (Huh-7) and kidney-derived human cell lines showed that $\mathrm{CatB} / \mathrm{CatL}$ dependence was independent of the target cell type. Similarly, CatB/CatL dependence was not impacted by the donor species, since EBOV-GP-driven entry into human, non-human primate and fruit bat cell lines was CatB/CatL-dependent, confirming a previous study (Hoffmann et al., 2016). However, these experiments revealed that Calu-3 cell entry driven by EBOV-GP and all other filovirus GPs studied was inefficient. This finding was remarkable for two reasons: First, EBOV exhibits a broad cell tropism and EBOV-GP is known to mediate entry 
276 into a diverse panel of cell lines, with only lymphoid cells being refractory for incompletely 277 understood reasons (Dube et al., 2010; Wool-Lewis and Bates, 1998; Yang et al., 1998). Second, 278 Calu-3 cells have previously been shown to express very low levels of endogenous CatL, which 279 were incompatible with CatL-dependent entry of MERS-CoV into these cells (Park et al., 2016). 280 This finding raised the question whether the low endogenous CatL expression might also limit

EBOV-GP-driven entry into these cells.

We confirmed low CatL expression in Calu-3 cells and equipped these cells with an expression cassette for CatL in order to unravel whether CatL expression limits EBOV-GP dependent entry. In parallel, we examined whether directed expression of the filovirus entry factors NPC1, Axl and DC-SIGN increased entry efficiency, which would suggest that attachment (DC-SIGN, Axl) or receptor engagement (NPC1) limits entry into Calu-3 cells. Finally, cells were equipped with CatB as control. We found that both directed expression of DC-SIGN and CatL can promote Calu-3 cell entry driven by filoviral GPs. However, the entry promoting effect of these factors was dependent on the viral GP studied. Expression of DCSIGN, a calcium-dependent lectin that binds to glycans on GP and promotes viral attachment to cells (Alvarez et al., 2002; Simmons et al., 2003), markedly increased entry driven by EBOV-GP, SUDV-GP and MARV-GP. Moreover, DC-SIGN expression moderately augmented entry driven by RESTV-GP and TAFV-GP, suggesting that viral attachment limits Calu-3 cell entry driven by most if not all GPs tested. In comparison, directed expression of CatL markedly increased MARV-GP- and moderately augmented EBOV-GP-, SUDV-GP-, TAFV-GP-, RESTV-GPdriven entry. These findings are largely in keeping with a previous report suggesting a pronounced contribution of CatL to RESTV- and MARV-GP-driven entry (Misasi et al., 2012) and demonstrate that priming can also limit GP-driven entry into Calu-3 cells. Finally, it is 


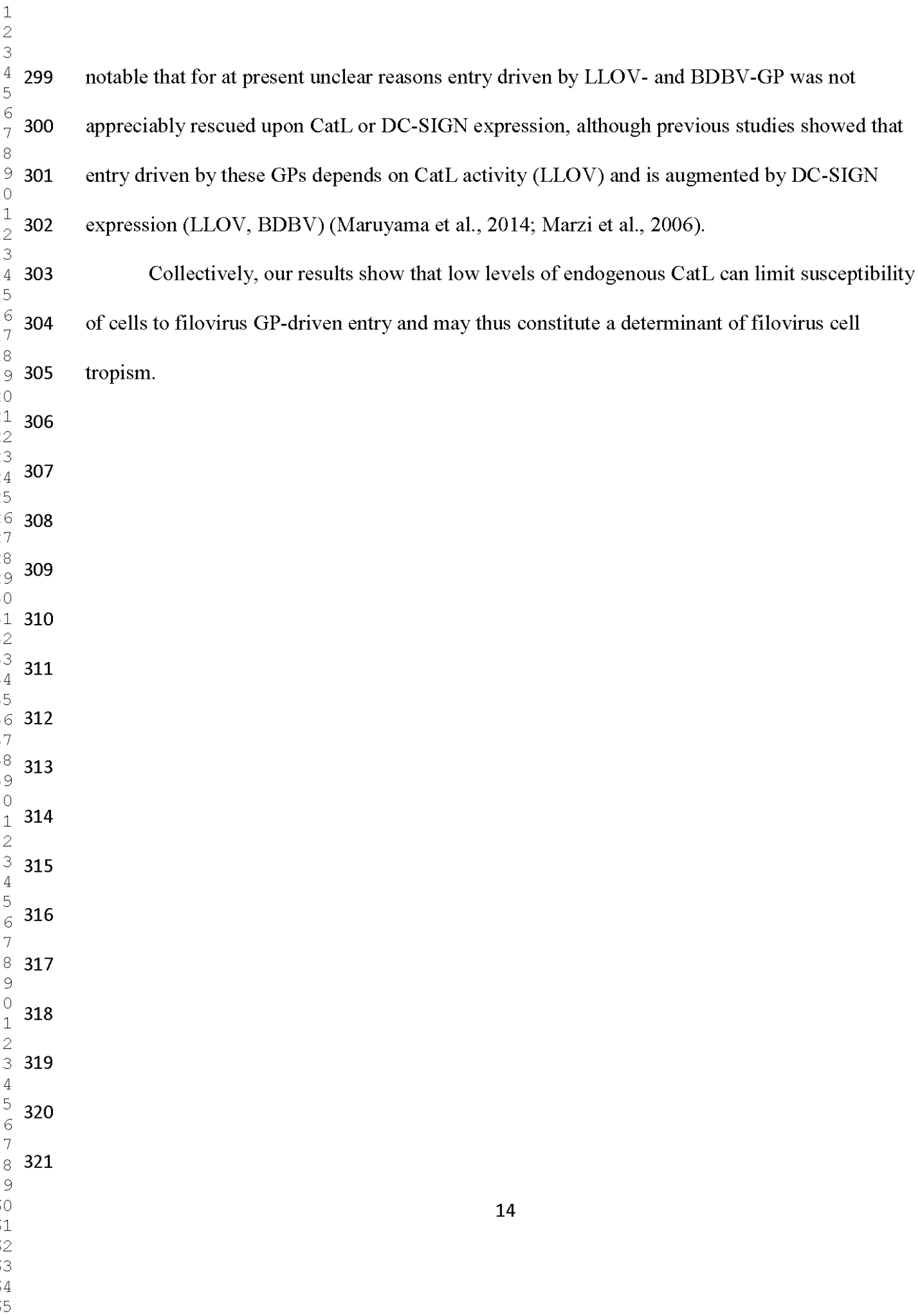




\section{Materials and methods}

\subsection{Cell culture}

Vero E6 (African green monkey kidney cells), 293T (human kidney cells), Huh-7 (human liver cells), A549 (human lung adenocarcinoma cells), Calu-3 (human bronchial adenocarcinoma cells) and EpoNi/22.1 (Buettikofer's epauletted fruit bat kidney cells) were incubated in a humidified atmosphere at $37^{\circ} \mathrm{C}$ and $5 \% \mathrm{CO}_{2}$. Vero E6, 293T, EpoNi/22.1, and Huh7 cell lines were maintained in Dulbecco's modified Eagle medium (DMEM, PAN-Biotech), A549 cells were grown in DMEM: Nutrient Mixture F-12 + GlutaMax medium (Gibco) and Calu-3 cells were maintained in minimum essential medium (MEM, Gibco). All media were supplemented with $10 \%$ fetal bovine serum (Biochrom) and $100 \mathrm{U} / \mathrm{ml}$ of penicillin and $0.1 \mathrm{mg} / \mathrm{ml}$ of streptomycin (PAN-Biotech). Calu-3 cells stably expressing cMYC-tagged CatB (GenBank: XM_017013097.2), CatL (GenBank: XM_005251716.4), DC-SIGN (GenBank: AB527563.1), NPC1 (GenBank: AB971140.1), and Axl (GenBank: BC032229.1) were generated using retroviral transduction and vector pQCXIP. For further cultivation, cells were maintained in MEM supplemented with $0.5 \mu \mathrm{g} / \mathrm{ml}$ of puromycin, $10 \%$ fetal bovine serum, and $1 \%$ of penicillinstreptomycin. All cells were detached by either resuspension in fresh culture medium (293T cells) or by the use of trypsin/EDTA (PAN-Biotech) for further subcultivation and seeding.

\subsection{Viruses}

We employed a previously described recombinant VSV (recVSV) containing a dual reporter consisting of eGFP and firefly luciferase (fLuc) that is located between the open reading frames 
345 for VSV-G and VSV-L(Gonzalez-Hernandez et al., 2018). Similarly, a chimeric VSV was used 346 in which the genetic information for VSV-G was replaced by that of EBOV-GP (Gonzalez347 Hernandez et al., 2018). All viruses were propagated on Vero E6 cells.

348 349 350 351

\subsection{Plasmids}

Expression plasmids encoding for the envelope glycoproteins of EBOV, BDBV, TAFV, RESTV, SUDV, MARV, LLOV and VSV have been described previously (Hoffmann et al., 2016). The plasmids required for the trVLP system (Watt et al., 2014) and VP30-luciferase containing particles have also been described before (Hoffmann et al., 2017). For the generation of CatB and CatL encoding retroviral vectors, the respective open reading frames were amplified by PCR from cDNA prepared from A549 cells and inserted into the vector pQCXIP using NotI and BamHI restriction enzymes. The same strategy was applied for generation of NPC1, DC-SIGN and Axl encoding vectors, cDNA prepared from Huh-7 cells (NPC1) and previously described expressions plasmids (DC-SIGN, Axl, (Pohlmann et al., 2001); (Watt et al., 2014)) were used as PCR template. The integrity of all PCR amplified sequences was confirmed by automated sequence analysis.

\subsection{Production of rhabdoviral pseudotypes}

The pseudotypes were generated and used for transduction as described previously (Hoffmann et al., 2016). Briefly, 293 T cells were seeded in 6-well plates and transfected using calcium phosphate precipitation with plasmids encoding the viral glycoproteins under study or empty 
1

5

plasmid (pCAGGS) as a negative control. At $18 \mathrm{~h}$ post transfection, the cells were inoculated at a multiplicity of infection (MOI) of 3 with a replication-deficient VSV, in which the ORF for VSV-G had been exchanged by two separate ORFs for eGFP and firefly luciferase (fLuc) (Berger Rentsch and Zimmer, 2011) (kindly provided by G. Zimmer). After $1 \mathrm{~h}$ incubation at $37^{\circ} \mathrm{C}$, the cells were washed with PBS and incubated for $1 \mathrm{~h}$ with a 1:1,000 dilution of I1 (an anti-VSV-G mouse hybridoma supernatant from CRL-2700; American Type Culture Collection) to neutralize residual input virus. Finally, fresh culture medium was added to the cells. At 18 to $20 \mathrm{~h}$ post transduction, supernatants were collected, clarified from cell debris by centrifugation at $4,700 \times \mathrm{g}$ for $10 \mathrm{~min}$, aliquoted, and stored at $-80^{\circ} \mathrm{C}$ until use.

\subsection{Production of MLV pseudotypes}

Transduction vectors based on murine leukemia virus (MLV, MLVpp) were generated according to an established protocol (Wrensch et al., 2014) with slight modifications. First, 293T cells grown in 6-well plates were transfected with MLV-Gag/Pol (4 $\mu$ g), MLV-Luc (6 $\mu g$ ) (Wrensch et al., 2014)and expression plasmid for the respective glycoprotein ( $4 \mu \mathrm{g})$. At 16 h post transfection, the cell culture supernatant was replaced by fresh culture medium. $48 \mathrm{~h}$ later, supernatants were collected and clarified from debris by centrifugation $(4,000 \times \mathrm{g}, 10 \mathrm{~min})$.

4.6. Production of EBOV-like particles 
389 Generation of EBOV-like particles containing VP30-Luc or the tetracistronic transcription and 390 replication competent EBOV minigenome (trVLP) was performed according to previously 391 published protocols(Hoffmann et al., 2017; Hoffmann et al., 2018; Watt et al., 2014):

392 Briefly, for production of VP30-Luc particles, 293T cells were grown in T-75 cell culture flasks 393 and transfected with expression plasmids for EBOV-NP $(1 \mu \mathrm{g})$, EBOV-VP35 $(1 \mu \mathrm{g}), \mathrm{EBOV}-\mathrm{L}$ $394(8.3 \mu \mathrm{g})$, EBOV-VP40 $(2 \mu \mathrm{g})$, EBOV-GP or VSV-G $(2 \mu \mathrm{g})$, and EBOV-VP30-Luc $(12.5 \mu \mathrm{g})$. At $39516 \mathrm{~h}$ post transfection, the cell culture supernatant was replaced by fresh culture medium. $24 \mathrm{~h}$

later, supernatants were collected, clarified from debris by centrifugation $(4,000 \times \mathrm{g}, 10 \mathrm{~min})$ and subsequently concentrated employing Vivaspin concentrator columns (molecular mass cutoff $=$ $30,000 \mathrm{kDa}$, Sartorius). Finally, volumes were equilibrated to $500 \mu \mathrm{l}$ by addition of DMEM. For production of EBOV trVLPs, $293 \mathrm{~T}$ cells were grown in 6-well plates and transfected with expression plasmids for EBOV-NP (125 ng), EBOV-VP35 (125 ng), EBOV-VP30 (75 ng) EBOV-L $(1 \mu \mathrm{g}), \mathrm{p} 4 \mathrm{cis}-\mathrm{vRNA}-\mathrm{RLuc}(250 \mathrm{ng})$ and T7-polymerase $(250 \mathrm{ng})$. At $16 \mathrm{~h}$ post transfection, the cell culture supernatant was replaced by fresh culture medium. $48 \mathrm{~h}$ later, supernatants were collected and clarified from debris by centrifugation $(4,000 \times \mathrm{g}, 10 \mathrm{~min})$.

\subsection{Transduction/Infection of cell lines and its inhibition with CatB and CatL inhibitors}

For transduction and infection, target cells were seeded in 96-well plates. At $24 \mathrm{~h}$ after seeding, medium was removed and cells were inoculated with equal volumes of transduction vectors (VSVpp, MLVpp, VP30-luc particles or trVLPs) or recVSV (MOI of 0.1). If required, cells were previously treated with inhibitor or antibody (see below). In case of transduction using EBOVlike particles based on the trVLP system, target cells were transfected with expression plasmids 

4 5 6 8

for EBOV-NP, -VP30, -VP35, -L and DC-SIGN prior $(24 \mathrm{~h})$ to transduction. In order to quantify transduction/infection efficiencies, firefly (fLuc, VSVpp, recVSV, MLVpp, VP30-Luc particles) or Renilla luciferase (rLuc, trVLP particles) activities in cell lysates were measured 24 (VSVpp, recVSV, VP30-Luc) or $48 \mathrm{~h}$ (MLVpp, trVLP) post transduction. For this, cell culture medium was aspirated, $50 \mu \mathrm{L}$ of cell culture lysis reagent (Promega) was added and cells incubated for 30 min at room temperature. Lysates were then transferred to a white, opaque-walled 96-well plate (Thermo Fisher Scientific), luciferin (fLuc; Beetle-Juice, PJK) or coelenterazine (rLuc; SigmaAldrich) was added and luciferase activity was measured in a microplate reader (Hidex Sense Microplate Reader) using the PlateReader Software (version 0.5.41.0, Hidex).

\subsection{Treatment of target cells with cathepsin inhibitors or anti-DC-SIGN antibody}

To investigate entry inhibition by MDL28170 (Tocris), E-64d (Tocris) or DC-SIGN antibody (120526, NIH-AIDS Research and Reference Reagent Program), the inhibitors or medium was added to target cells and the cells were incubated for $3 \mathrm{~h}$ (MDL28170/E-64d) or $1 \mathrm{~h}$ ( DC-SIGN antibody) before transduction/infection was performed

\subsection{Quantification of CatB/CatL $m R N A$ expression}

Total RNA from 293T, Huh-7, and Calu-3 cell lines was extracted using the RNeasy Mini Kit (Qiagen) following the manufacturer instructions. Afterwards, cDNA was produced using the 433 SuperScript III First Strand Synthesis System (Invitrogen) with random hexamers. To determine the copy numbers CatB/CatL mRNA expression, quantitative PCR was performed using the 
435 QuantiTect SYBR Green PCR Kit (Qiagen), $1 \mu 1$ of cDNA as template and the following 436 primers: Cathepsin B 5'-TACAGCCCGACCTACAAACA-3', 5'-

437 CCATGATGTCCTTCTCGCTA-3'; Cathepsin L 5'-GCAGGTCATGAGTCCTTCCT-3', 5'-

438 CTTTACGTAGCCACCCATGC-3'; $\beta$-actin 5'-GGCTCCCAGCACAATGAAGA-3', 5'439 GGAGCCGCCGATCCA-3'. As standard, serial dilutions of expression plasmids for CatB, CatL, 440 and $\beta$-actin were subjected to PCR analysis. Ct values were determined using the Rotor-Gene Q 441 device along with the Rotor-Gene Q software (Qiagen) and used to calculate their respective copy numbers. Further, copy numbers for CatB and CatL were normalized against that of B-actin.

To detect expression of entry and attachment factors in stably transduced Calu-3 cell lines, the cells were lysed using $200 \mu \mathrm{L} 2 \mathrm{x}$ sodium dodecyl sulfate (SDS)-containing lysis buffer (50 mM Tris (pH 6.8), 10\% glycerol, 2\% SDS, 5\% $\beta$-mercaptoethanol, $0.1 \%$ bromophenol blue, $1 \mathrm{mM}$ EDTA), and boiled for $15 \mathrm{~min}$ at $95^{\circ} \mathrm{C}$. Subsequently, samples were separated by SDS-PAGE and blotted onto nitrocellulose membranes (GE Healthcare Life Sciences; $0.2 \mu \mathrm{m}$ ). Afterwards, membranes were blocked for $30 \mathrm{~min}$ in PBS containing 5\% milk powder and $0.1 \%$ Tween 20 . Finally, expression of cMYC tagged CatL, CatB, DC-SIGN, NPC1 and Axl was determined using undiluted supernatants of a hybridoma cell line that secretes anti-MYC antibody (9E10). Bound antibodies were detected using a horseradish peroxidase linked anti-mouse antibody (Dianova) at a 1:5,000 dilution. Signals were detected using the ChemoCam imaging system along with the ChemoStarProfessional software (Intas). 


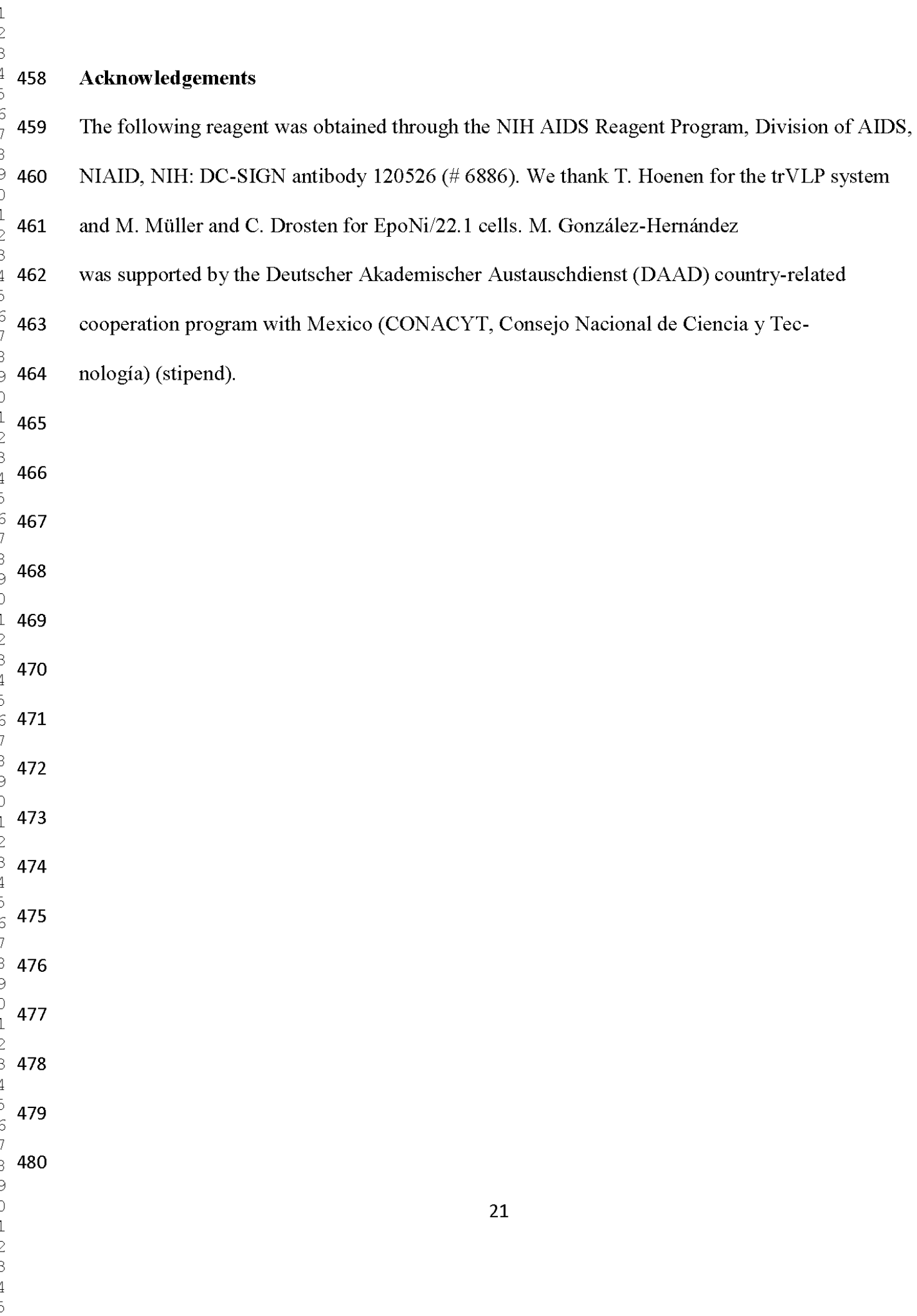




\section{References}

Alvarez, C.P., Lasala, F., Carrillo, J., Muniz, O., Corbi, A.L., Delgado, R., 2002. C-type lectins DC-SIGN and L-SIGN mediate cellular entry by Ebola virus in cis and in trans. J Virol 76, 6841-6844.

Bale, S., Liu, T., Li, S., Wang, Y., Abelson, D., Fusco, M., Woods, V.L., Jr., Saphire, E.O., 2011. Ebola virus glycoprotein needs an additional trigger, beyond proteolytic priming for membrane fusion. PLoS Negl Trop Dis 5, e1395.

Berger Rentsch, M., Zimmer, G., 2011. A vesicular stomatitis virus replicon-based bioassay for the rapid and sensitive determination of multi-species type I interferon. PLoS One 6, e25858.

Brana, C., Benham, C.D., Sundstrom, L.E., 1999. Calpain activation and inhibition in organotypic rat hippocampal slice cultures deprived of oxygen and glucose. Eur J Neurosci 11 , 2375-2384.

Brecher, M., Schornberg, K.L., Delos, S.E., Fusco, M.L., Saphire, E.O., White, J.M., 2012. Cathepsin cleavage potentiates the Ebola virus glycoprotein to undergo a subsequent fusionrelevant conformational change. J Virol 86, 364-372.

Carette, J.E., Raaben, M., Wong, A.C., Herbert, A.S., Obernosterer, G., Mulherkar, N., Kuehne, A.I., Kranzusch, P.J., Griffin, A.M., Ruthel, G., Dal Cin, P., Dye, J.M., Whelan, S.P., Chandran, K., Brummelkamp, T.R., 2011. Ebola virus entry requires the cholesterol transporter Niemann-Pick C1. Nature 477, 340-343.

Centers for Disease Control and Prevention, U., 2018. 40 Years of Ebola Virus Disease around the World.

Chandran, K., Sullivan, N.J., Felbor, U., Whelan, S.P., Cunningham, J.M., 2005. Endosomal proteolysis of the Ebola virus glycoprotein is necessary for infection. Science 308, 1643-1645.

Cote, M., Misasi, J., Ren, T., Bruchez, A., Lee, K., Filone, C.M., Hensley, L., Li, Q., Ory, D., Chandran, K., Cunningham, J., 2011. Small molecule inhibitors reveal Niemann-Pick C1 is essential for Ebola virus infection. Nature 477, 344-348.

Dietzel, E., Schudt, G., Krahling, V., Matrosovich, M., Becker, S., 2017. Functional Characterization of Adaptive Mutations during the West African Ebola Virus Outbreak. J Virol 91.

Dube, D., Schornberg, K.L., Shoemaker, C.J., Delos, S.E., Stantchev, T.S., Clouse, K.A., Broder, C.C., White, J.M., 2010. Cell adhesion-dependent membrane trafficking of a binding partner for the ebolavirus glycoprotein is a determinant of viral entry. Proc Natl Acad Sci U S A 107 , $16637-16642$.

Gewies, A., Grimm, S., 2003. Cathepsin-B and cathepsin-L expression levels do not correlate with sensitivity of tumour cells to TNF-alpha-mediated apoptosis. Br J Cancer 89, 1574-1580.

Gnirss, K., Kuhl, A., Karsten, C., Glowacka, I., Bertram, S., Kaup, F., Hofmann, H., Pohlmann, S., 2012. Cathepsins B and L activate Ebola but not Marburg virus glycoproteins for efficient entry into cell lines and macrophages independent of TMPRSS2 expression. Virology 424, 310.

Gonzalez-Hernandez, M., Hoffmann, M., Brinkmann, C., Nehls, J., Winkler, M., Schindler, M., Pohlmann, S., 2018. A GXXXA Motif in the Transmembrane Domain of the Ebola Virus Glycoprotein Is Required for Tetherin Antagonism. J Virol 92.

Hoffmann, M., Crone, L., Dietzel, E., Paijo, J., Gonzalez-Hernandez, M., Nehlmeier, I., Kalinke, U., Becker, S., Pohlmann, S., 2017. A Polymorphism within the Internal Fusion Loop of the Ebola Virus Glycoprotein Modulates Host Cell Entry. J Virol 91. 
Hoffmann, M., Gonzalez Hernandez, M., Berger, E., Marzi, A., Pohlmann, S., 2016. The Glycoproteins of All Filovirus Species Use the Same Host Factors for Entry into Bat and Human Cells but Entry Efficiency Is Species Dependent. PLoS One 11, e0149651.

Hoffmann, M., Nehlmeier, I., Brinkmann, C., Krahling, V., Behner, L., Moldenhauer, A.S., Kruger, N., Nehls, J., Schindler, M., Hoenen, T., Maisner, A., Becker, S., Pohlmann, S., 2018. Tetherin inhibits Nipah virus but not Ebola virus replication in fruit bat cells. J Virol.

Jemielity, S., Wang, J.J., Chan, Y.K., Ahmed, A.A., Li, W., Monahan, S., Bu, X., Farzan, M., Freeman, G.J., Umetsu, D.T., Dekruyff, R.H., Choe, H., 2013. TIM-family proteins promote infection of multiple enveloped viruses through virion-associated phosphatidylserine. PLoS Pathog 9, e1003232.

Kaletsky, R.L., Simmons, G., Bates, P., 2007. Proteolysis of the Ebola virus glycoproteins enhances virus binding and infectivity. J Virol 81, 13378-13384.

Kondratowicz, A.S., Lennemann, N.J., Sinn, P.L., Davey, R.A., Hunt, C.L., Moller-Tank, S., Meyerholz, D.K., Rennert, P., Mullins, R.F., Brindley, M., Sandersfeld, L.M., Quinn, K., Weller, M., McCray, P.B., Jr., Chiorini, J., Maury, W., 2011. T-cell immunoglobulin and mucin domain 1 (TIM-1) is a receptor for Zaire Ebolavirus and Lake Victoria Marburgvirus. Proc Natl Acad Sci U S A 108, 8426-8431.

Kuhn, J.H., 2017. Guide to the Correct Use of Filoviral Nomenclature. Curr Top Microbiol Immunol 411, 447-460.

Lo, T.Q., Marston, B.J., Dahl, B.A., De Cock, K.M., 2017. Ebola: Anatomy of an Epidemic. Annu Rev Med 68, 359-370.

Mandl, J.N., Schneider, C., Schneider, D.S., Baker, M.L., 2018. Going to Bat(s) for Studies of Disease Tolerance. Front Immunol 9, 2112.

Martin, B., Hoenen, T., Canard, B., Decroly, E., 2016. Filovirus proteins for antiviral drug discovery: A structure/function analysis of surface glycoproteins and virus entry. Antiviral Res $135,1-14$.

Martinez, O., Johnson, J., Manicassamy, B., Rong, L., Olinger, G.G., Hensley, L.E., Basler, C.F., 2010. Zaire Ebola virus entry into human dendritic cells is insensitive to cathepsin $\mathrm{L}$ inhibition. Cell Microbiol 12, 148-157.

Maruyama, J., Miyamoto, H., Kajihara, M., Ogawa, H., Maeda, K., Sakoda, Y., Yoshida, R., Takada, A., 2014. Characterization of the envelope glycoprotein of a novel filovirus, lloviu virus. J Virol 88, 99-109.

Marzi, A., Reinheckel, T., Feldmann, H., 2012. Cathepsin B \& L are not required for ebola virus replication. PLoS Negl Trop Dis 6, e1923.

Marzi, A., Wegele, A., Pohlmann, S., 2006. Modulation of virion incorporation of Ebolavirus glycoprotein: effects on attachment, cellular entry and neutralization. Virology 352, 345-356.

Misasi, J., Chandran, K., Yang, J.Y., Considine, B., Filone, C.M., Cote, M., Sullivan, N., Fabozzi, G., Hensley, L., Cunningham, J., 2012. Filoviruses require endosomal cysteine proteases for entry but exhibit distinct protease preferences. J Virol 86, 3284-3292.

Moller-Tank, S., Kondratowicz, A.S., Davey, R.A., Rennert, P.D., Maury, W., 2013. Role of the phosphatidylserine receptor TIM-1 in enveloped-virus entry. J Virol 87, 8327-8341.

Olival, K.J., Hayman, D.T., 2014. Filoviruses in bats: current knowledge and future directions. Viruses 6, 1759-1788.

Pager, C.T., Craft, W.W., Jr., Patch, J., Dutch, R.E., 2006. A mature and fusogenic form of the Nipah virus fusion protein requires proteolytic processing by cathepsin L. Virology 346, 251257. 
Park, J.E., Li, K., Barlan, A., Fehr, A.R., Perlman, S., McCray, P.B., Jr., Gallagher, T., 2016. Proteolytic processing of Middle East respiratory syndrome coronavirus spikes expands virus tropism. Proc Natl Acad Sci U S A 113, 12262-12267.

Pohlmann, S., Baribaud, F., Lee, B., Leslie, G.J., Sanchez, M.D., Hiebenthal-Millow, K., Munch, J., Kirchhoff, F., Doms, R.W., 2001. DC-SIGN interactions with human immunodeficiency virus type 1 and 2 and simian immunodeficiency virus. J Virol 75, 4664-4672.

Quinn, K., Brindley, M.A., Weller, M.L., Kaludov, N., Kondratowicz, A., Hunt, C.L., Sinn, P.L., McCray, P.B., Jr., Stein, C.S., Davidson, B.L., Flick, R., Mandell, R., Staplin, W., Maury, W., Chiorini, J.A., 2009. Rho GTPases modulate entry of Ebola virus and vesicular stomatitis virus pseudotyped vectors. J Virol 83, 10176-10186.

Saeed, M.F., Kolokoltsov, A.A., Albrecht, T., Davey, R.A., 2010. Cellular entry of ebola virus involves uptake by a macropinocytosis-like mechanism and subsequent trafficking through early and late endosomes. PLoS Pathog 6, e1001110.

Sanchez, A., 2007. Analysis of filovirus entry into vero e6 cells, using inhibitors of endocytosis, endosomal acidification, structural integrity, and cathepsin (B and L) activity. J Infect Dis 196 Supp1 2, S251-258.

Schornberg, K., Matsuyama, S., Kabsch, K., Delos, S., Bouton, A., White, J., 2006. Role of endosomal cathepsins in entry mediated by the Ebola virus glycoprotein. J Virol 80, 41744178 .

Shimojima, M., Takada, A., Ebihara, H., Neumann, G., Fujioka, K., Irimura, T., Jones, S., Feldmann, H., Kawaoka, Y., 2006. Tyro3 family-mediated cell entry of Ebola and Marburg viruses. J Virol 80, 10109-10116.

Simmons, G., Gosalia, D.N., Rennekamp, A.J., Reeves, J.D., Diamond, S.L., Bates, P., 2005. Inhibitors of cathepsin L prevent severe acute respiratory syndrome coronavirus entry. Proc Natl Acad Sci U S A 102, 11876-11881.

Simmons, G., Reeves, J.D., Grogan, C.C., Vandenberghe, L.H., Baribaud, F., Whitbeck, J.C., Burke, E., Buchmeier, M.J., Soilleux, E.J., Riley, J.L., Doms, R.W., Bates, P., Pohlmann, S., 2003. DC-SIGN and DC-SIGNR bind ebola glycoproteins and enhance infection of macrophages and endothelial cells. Virology 305, 115-123.

Takada, A., Robison, C., Goto, H., Sanchez, A., Murti, K.G., Whitt, M.A., Kawaoka, Y., 1997. A system for functional analysis of Ebola virus glycoprotein. Proc Natl Acad Sci U S A 94, 14764-14769.

Wang, Z., Li, J., Fu, Y., Zhao, Z., Zhang, C., Li, N., Li, J., Cheng, H., Jin, X., Lu, B., Guo, Z., Qian, J., Liu, L., 2018. A Rapid Screen for Host-Encoded miRNAs with Inhibitory Effects against Ebola Virus Using a Transcription- and Replication-Competent Virus-Like Particle System. Int J Mol Sci 19.

Watt, A., Moukambi, F., Banadyga, L., Groseth, A., Callison, J., Herwig, A., Ebihara, H., Feldmann, H., Hoenen, T., 2014. A novel life cycle modeling system for Ebola virus shows a genome length-dependent role of VP24 in virus infectivity. J Virol 88, 10511-10524.

WHO, 2018. Ebola situation reports: Democratic Republic of the Congo. World Health Organization.

Wool-Lewis, R.J., Bates, P., 1998. Characterization of Ebola virus entry by using pseudotyped viruses: identification of receptor-deficient cell lines. J Virol 72, 3155-3160.

Wrensch, F., Winkler, M., Pohlmann, S., 2014. IFITM proteins inhibit entry driven by the MERS-coronavirus spike protein: evidence for cholesterol-independent mechanisms. Viruses 6, 3683-3698 
1

4618 Yang, Z., Delgado, R., Xu, L., Todd, R.F., Nabel, E.G., Sanchez, A., Nabel, G.J., 1998. Distinct 619 cellular interactions of secreted and transmembrane Ebola virus glycoproteins. Science 279, 620 1034-1037. 


\section{Figure legends}

643
Fig. 1. EBOV-GP-driven entry into $293 \mathrm{~T}$ cells requires CatB/CatL activity irrespective of the transduction system. 293T were seeded in 96-well plates and pre-incubated with DMSO or the indicated concentrations of E-64d or MDL28170 and then transduced with equal volumes of (A) single-cycle VSV particles (VSVpp) pseudotyped with the indicated GPs or no glycoprotein as negative control; (B) replication-competent VSV or VSV-chimera encoding EBOV-GP instead of VSV-G at a MOI of 0.1 ; (C) MLV particles pseudotyped with the indicated glycoproteins or no glycoprotein; (D) EBOV-like particles containing VP30-luciferase and bearing EBOV-GP, VSVG or no glycoprotein; (E) EBOV-like particles generated in the trVLP system. Luciferase activities in cell lysates were determined at $24 \mathrm{~h}$ post transduction as indicator of transduction efficiency. In panels A-D, transduction mediated by EBOV-GP and VSV-G is shown relative to transduction mediated by control particles bearing no glycoprotein, which was set as 1 . In panel $\mathrm{E}$, transduction by particles produced in the absence of the viral polymerase, L, were set as 1 . The average of three independent experiments is shown in each panel. Error bars indicate standard error of the mean (SEM).

Fig. 2. EBOV-GP-driven entry is CatB/CatL-dependent irrespective of the target cell line. Equal volumes of VSVpp bearing the indicated glycoproteins or bearing no glycoprotein were used for transduction of 293T, Huh-7, A549, Calu-3, Vero E6, and EpoNi/22.1 cell lines pre-incubated for $3 \mathrm{~h}$ with E-64d in the indicated concentrations or DMSO as control. At $24 \mathrm{~h}$ post transduction, luciferase activities in cell lysates were quantified as indicator of transduction efficiency. Transduction mediated by EBOV-GP and VSV-G is shown relative to transduction mediated by 
control particles bearing no glycoprotein, which was set as 1 . The average of four independent experiments conducted with separate pseudotype preparations is shown. Error bars indicate SEM.

Fig. 3. Calu-3 cells are largely resistant to entry driven by filoviral but not arena-, paramyxo-and rhabdoviral glycoproteins. Vero E6 and Calu-3 cells were transduced with equal volumes of VSVpp pseudotyped with the glycoproteins from diverse viruses (A) or pseudotyped with filovirus glycoproteins (B). Particles bearing no glycoprotein served as negative control. At $24 \mathrm{~h}$ post transduction, luciferase activities in cell lysates were quantified as indicator of transduction efficiency. Transduction mediated by the different glycoproteins is shown relative to transduction mediated by control particles bearing no glycoprotein, which was set as 1 . The average of three independent experiments is shown. Error bars indicate SEM.

Fig. 4. Directed expression of Cat L and DC-SIGN can rescue filovirus GP-driven entry into Calu-3 cells. (A) CatB, CatL and $\beta$-actin mRNA expression in 293T, Huh-7 and Calu-3 cells was quantified by quantitative RT-PCR. CatB and CatL copy numbers were plotted in relation to $\beta$ actin copy numbers for each cell line. Shown is a single representative experiment with triplicate samples, error bars indicate standard deviation (SD). The results were confirmed in a second independent experiment. (B) Calu-3 cells engineered to stably express CatB, CatL, DC-SIGN, NPC1, and Axl with a cMYC antigenic tag were subjected to immunoblot analysis using an anticMYC antibody. Detection of $\beta$-actin expression served as loading control. Similar results were obtained in a separate experiment. (C) Equal volumes of VSVpp bearing the indicated filovirus glycoproteins were used to transduce Calu-3 cells stably expressing the indicated entry and attachment factors. At $24 \mathrm{~h}$ post transduction, luciferase activities in cell lysates were quantified 
as indicator of transduction efficiency. Transduction driven by the different GPs in Calu-3 cells stably expressing the indicated cellular factors is shown relative to transduction of Calu-3 control cells which was set as 1 . The average of five independent experiments is shown. Error bars indicate SEM. (D) Calu-3 control cells and Calu-3 CatL-cMYC cells were incubated for $3 \mathrm{~h}$ with DMSO or $50 \mu \mathrm{M}$ of MDL28170 and transduced with same volume of VSV-pseudotyped with MARV-GP, VSV-G or bearing no glycoprotein. (E) Calu-3 control cells and Calu-3 DC-SIGNcMYC cells were pre-incubated for $1 \mathrm{~h}$ with $20 \mu \mathrm{g} / \mathrm{ml}$ of anti-DC-SIGN monoclonal antibody or an isotype matched control antibody and then transduced with equal volumes of VSV particles bearing the indicated glycoproteins or bearing no GP. For panels (D) and (E) luciferase activity was quantified at $24 \mathrm{~h}$ post transduction. Entry into Calu-3 CatL and Calu-3 DC-SIGN cells is shown relative to entry into Calu-3 control cells, which was set as 1 . The average of at least five independent experiments is shown. Error bars indicate SEM. 
Figure 1

Click here to download high resolution i mage

Figure 1

A)

MDL28170

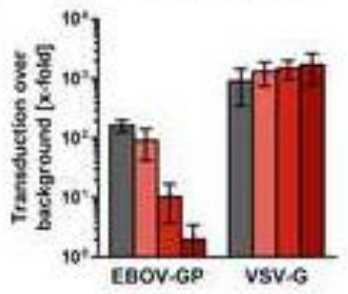

B)

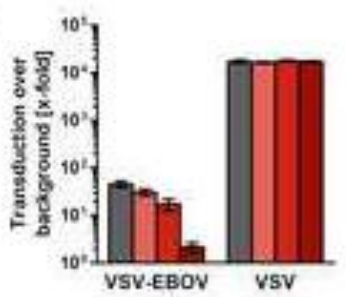

C)

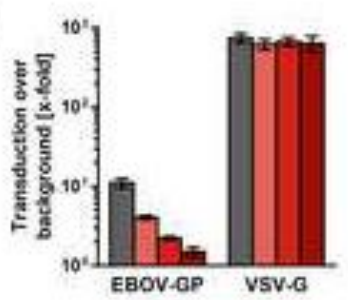

D)

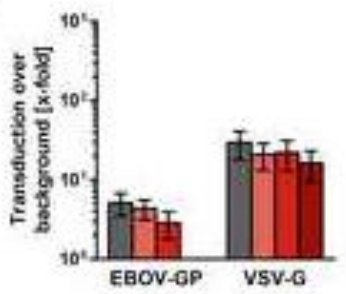

E)

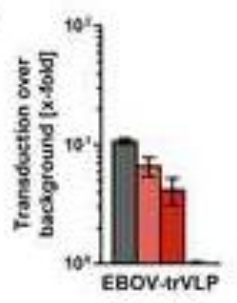

González Hernández et al., 2018

E-64d

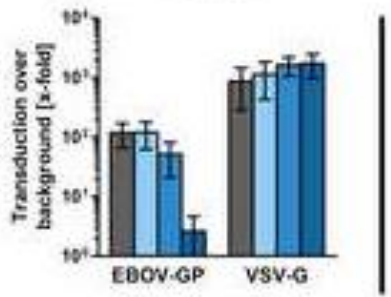

VSVpp

recVSV
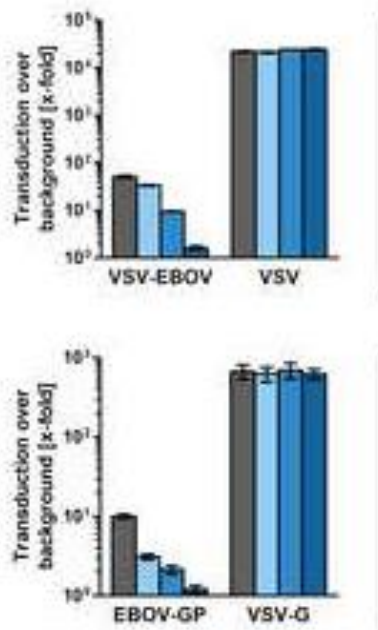

MLVpp

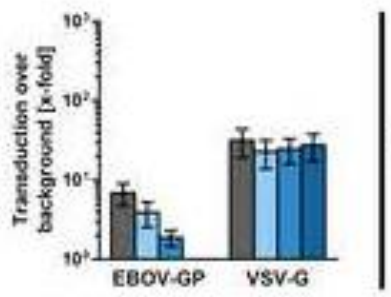

VP30-Luc

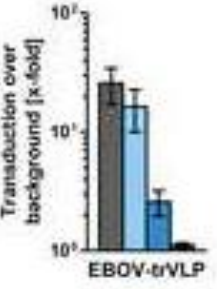

trVLP

DoMso $\frac{M D L 28170 / E-64 d}{\square 0.5 \mu M \text { D } 5 \mu \mathrm{M} \square 50 \mu \mathrm{M}}$ 
Figure 2

Click here to download high resolution i mage

Figure 2

$293 T$

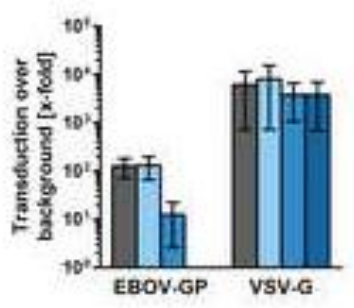

A549

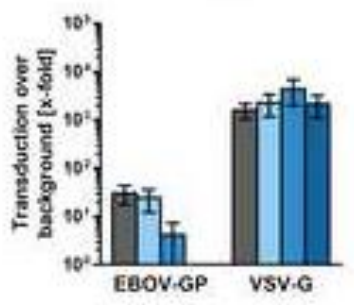

González Hernández et al., 2018

Huh-7

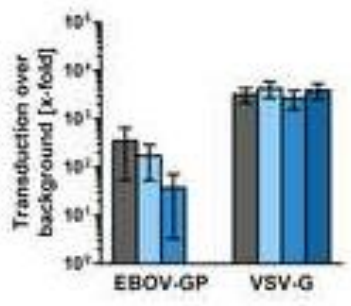

Calu-3

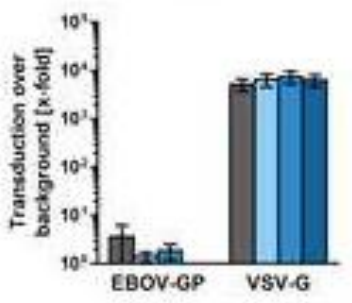

Vero E6

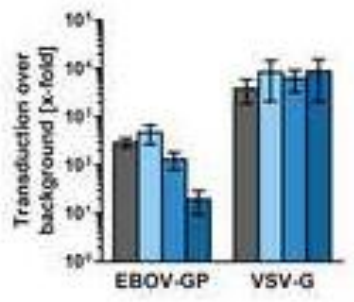

EpoNi/22.1

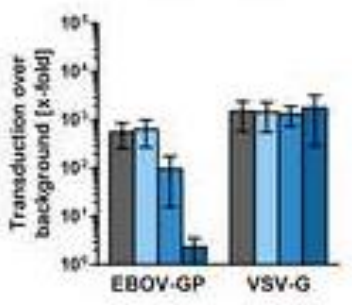

Domso $\frac{\text { E-64d }}{0.5 \mu \mathrm{M} \text { 回 } 5 \mu \mathrm{M} \text { 圆 } 50 \mu \mathrm{M}}$ 
Figure 3

Click here to download high resolution i mage

Figure 3

A)

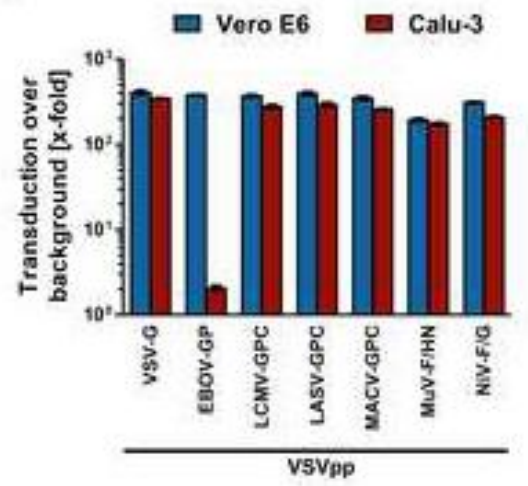

González Hernández et al., 2018

B)

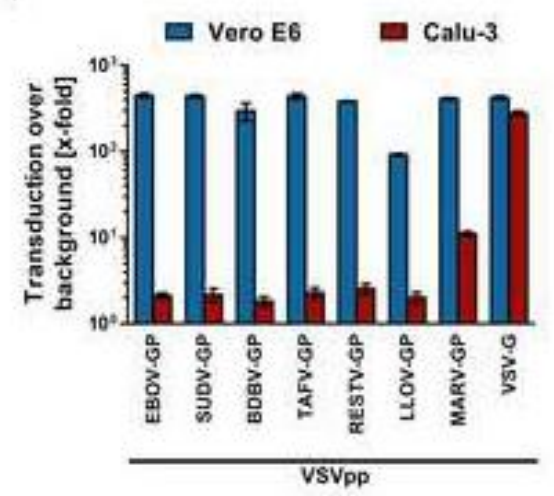


Figure 4

Click here to download high resolution i mage

Figure 4

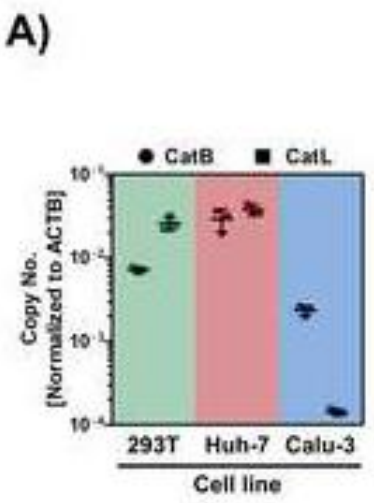

González Hernández et al., 2018

B)

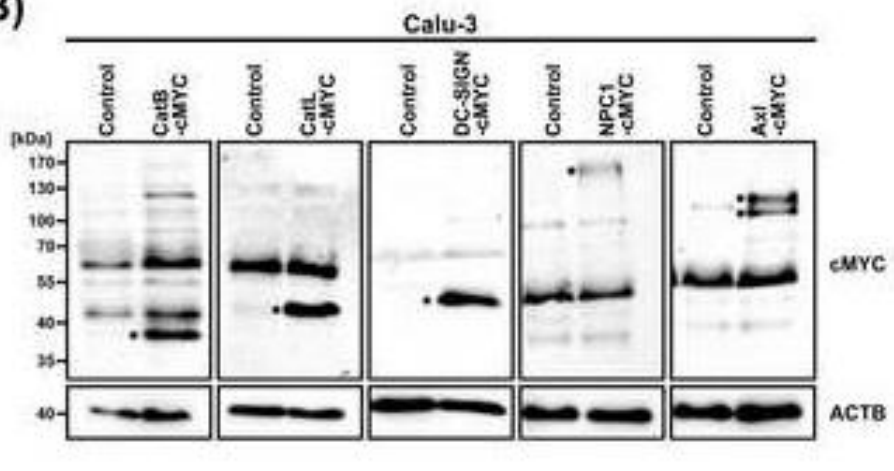

C)

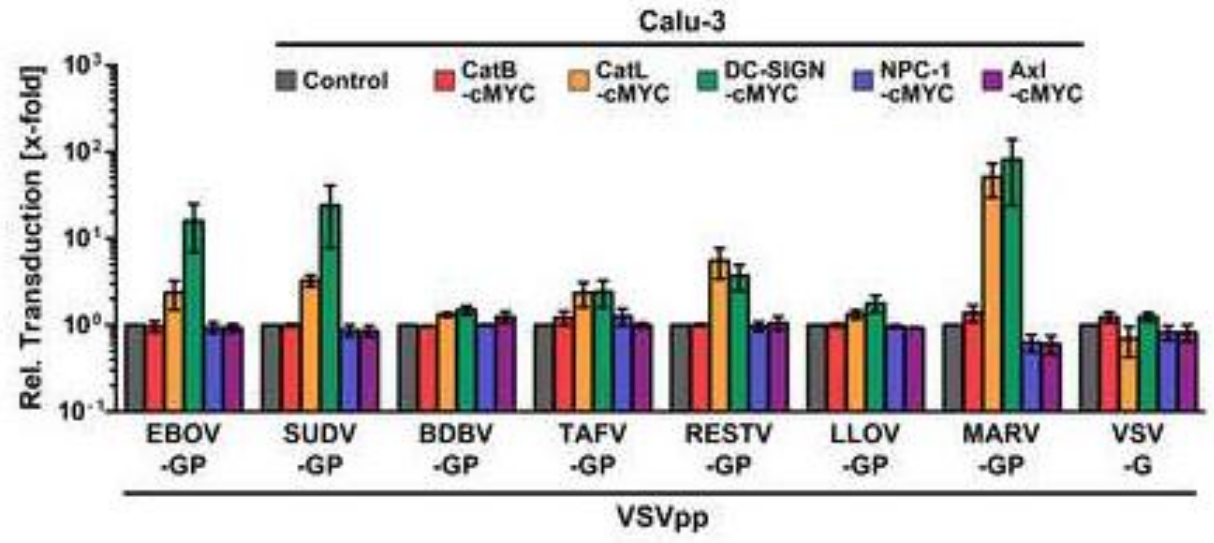

D)
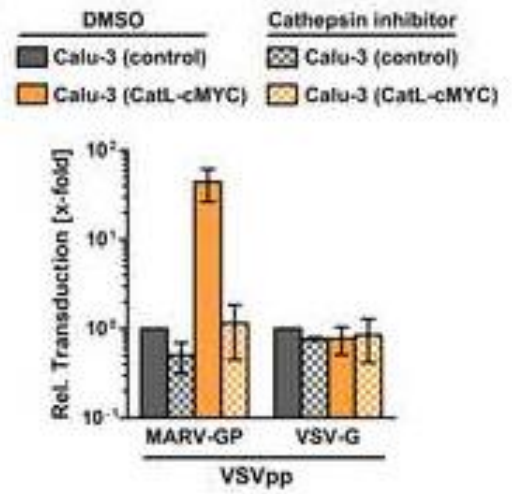

E)

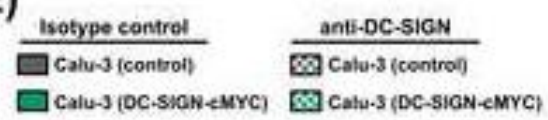

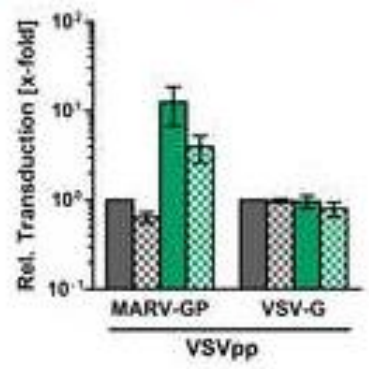




\subsection{Second manuscript}

\section{A GXXXA Motif in the Transmembrane Domain of the Ebola Virus Glycoprotein Is Required for Tetherin Antagonism.}

González-Hernández, M., Hoffmann, M., Brinkmann, C., Nehls, J., Winkler, M., Schindler, M., Pöhlmann, S.

Journal of Virology, 2018. 92(13):e00403-18. doi: 10.1128/JVI.00403-18.

Individual contributions:

I conducted the experiments that resulted in the data presented in figure 1 (panels BD), figure 2 (panels A-D), figure 3 (panels $A-C$ ) and figure 4 (panels $C, D$ and $E$ ). Furthermore, I analyzed the data that I generated, quantified the immunoblots blots and wrote parts of the manuscript. 


\section{A GXXXA Motif in the Transmembrane Domain of the Ebola Virus Glycoprotein Is Required for Tetherin Antagonism}

\author{
Michael Schindler,b,c @Stefan Pöhlmann \\ aInfection Biology Unit, German Primate Center, Göttingen, Germany \\ bInstitute of Medical Virology and Epidemiology of Viral Diseases, University Hospital Tübingen, Tübingen, \\ Germany \\ -Institute of Virology, Helmholtz Center Munich, German Research Center for Environmental Health, \\ Neuherberg, Germany
}

Mariana González-Hernández, ${ }^{a}$ Markus Hoffmann, a Constantin Brinkmann, a Julia Nehls, b,c Michael Winkler,

ABSTRACT The interferon-induced antiviral host cell protein tetherin can inhibit the release of several enveloped viruses from infected cells. The Ebola virus (EBOV) glycoprotein (GP) antagonizes tetherin, but the domains and amino acids in GP that are required for tetherin antagonism have not been fully defined. A GXXXA motif within the transmembrane domain (TMD) of EBOV-GP was previously shown to be important for GP-mediated cellular detachment. Here, we investigated whether this motif also contributes to tetherin antagonism. Mutation of the GXXXA motif did not impact GP expression or particle incorporation and only modestly reduced EBOV-GPdriven entry. In contrast, the GXXXA motif was required for tetherin antagonism in transfected cells. Moreover, alteration of the GXXXA motif increased tetherin sensitivity of a replication-competent vesicular stomatitis virus (VSV) chimera encoding EBOV-GP. Although these results await confirmation with authentic EBOV, they indicate that a GXXXA motif in the TMD of EBOV-GP is important for tetherin antagonism. Moreover, they provide the first evidence that GP can antagonize tetherin in the context of an infectious EBOV surrogate.

IMPORTANCE The glycoprotein (GP) of Ebola virus (EBOV) inhibits the antiviral host cell protein tetherin and may promote viral spread in tetherin-positive cells. However, tetherin antagonism by GP has so far been demonstrated only with virus-like particles, and it is unknown whether GP can block tetherin in infected cells. Moreover, a mutation in GP that selectively abrogates tetherin antagonism is unknown. Here, we show that a GXXXA motif in the transmembrane domain of EBOV-GP, which was previously reported to be required for GP-mediated cell rounding, is also important for tetherin counteraction. Moreover, analysis of this mutation in the context of vesicular stomatitis virus chimeras encoding EBOV-GP revealed that GPmediated tetherin counteraction is operative in infected cells. To our knowledge, these findings demonstrate for the first time that GP can antagonize tetherin in infected cells and provide a tool to study the impact of GP-dependent tetherin counteraction on EBOV spread.

KEYWORDS Ebola virus, glycoprotein, tetherin

- he interferon (IFN) system is a component of innate immunity and constitutes the

first line of defense against viral infection. The IFN response is characterized by three processes: the sensing of invading pathogens, the induction of signaling cascades, and the expression of IFN-stimulated genes (ISGs) (1-4). The entire spectrum of ISGs was first comprehensively characterized by Schoggins and colleagues (3), who showed that many of the 300 to 400 ISG-encoded proteins exert antiviral activity.
Received 8 March 2018 Accepted 13 April 2018 Accepted manuscript posted online 18 Aprill 2018

Citation González-Hernández M, Hoff́mann M Brinkmann C, Nehls J, Winkler M, Schindler M Pohimann S. 2018. A G)OO(A motif in the transmembrane domain of the Ebola virus glycoprotein is required for tetherin antagonism. J Virol 92:e00403-18. https://doi .org/10.1128/N/.00403-18

Editor Terence S. Dermody, University of Pittsburgh School of Medicine Copyright $\ominus 2018$ American Soclety for Microblology. All Rights Reserved. Address correspondence to Stefan Pöhlmann, spœehimann@dpz.eu. 
Understanding how ISG-encoded proteins inhibit viruses will provide not only important insights into how virus infection can be controlled by innate immunity but also a possible basis for novel antiviral strategies.

The host cell protein tetherin is upregulated by IFN (i.e., is encoded by an ISG) and is also constitutively expressed in certain cells and tissues $(5,6)$. Tetherin inhibits release of progeny particles from infected cells by forming a physical tether between the virus and host cell $(6,7)$. This activity critically depends on tetherin's particular domain organization: the protein harbors an N-terminal transmembrane domain (TMD) and a C-terminal glycosylphosphatidylinositol (GPI) anchor, which permit simultaneous insertion of tetherin into a viral and a cellular membrane. In response to the antiviral pressure imposed by tetherin, several viruses have evolved tetherin antagonists (8), including the HIV-1 protein Vpu (6), and most of these proteins block tetherin's antiviral activity by reducing tetherin levels at the site of viral budding, the plasma membrane (8).

The Ebola virus (EBOV), a member of the family Filoviridae, causes severe and frequently fatal disease in humans and nonhuman primates. The virus blocks signaling pathways of the IFN system via its VP24 and VP35 proteins (9). In addition, EBOV and other filoviruses counteract tetherin via their only virus-encoded surface protein, the viral glycoprotein (GP) (10-12). In this context, it should be noted that tetherin antagonism by GP has so far exclusively been demonstrated in cells releasing EBOV-like particles, and it is unknown if GP antagonizes tetherin in the context of EBOV infection and whether such antagonism is required for viral spread. In contrast to Vpu, EBOV-GP interferes with tetherin's antiviral activity without altering tetherin expression levels or cellular localization (12-14), and the mechanism underlying tetherin counteraction by EBOV-GP is largely unclear. Moreover, it is incompletely understood which domains in GP contribute to tetherin antagonism. Vande Burgt and colleagues reported a role for the glycan cap (15), and our previous work demonstrated that a single amino acid change in the receptor binding domain (RBD) can abrogate tetherin counteraction (10). In addition, the transmembrane domain (TMD) of EBOV-GP was shown to contribute to tetherin antagonism $(15,16)$, but the responsible amino acid motifs remain to be elucidated.

Here, we employed EBOV surrogate systems to investigate the contribution of a GXXXA motif in the TMD of EBOV-GP to tetherin antagonism. Although confirmation with infectious EBOV is pending and conclusions are thus tentative, our results indicate that the GXXXA motif, which was previously reported to be important for GP-mediated cell detachment (17), is also required for tetherin antagonism. Moreover, these results provide the first evidence that GP can antagonize tetherin in the context of infected cells.

\section{RESULTS}

Mutation of the GXXXA motif in the EBOV-GP TMD is compatible with robust GP expression and only modestly reduces GP-driven host cell entry. To address the role of the GXXXA motif in the EBOV-GP TMD in tetherin antagonism, we first changed the GXXXA motif to LXXXL using PCR-based mutagenesis (Fig. 1A). Next, we investigated GP expression and incorporation into viral particles, employing transiently transfected 293T cells. We found that EBOV-GP wild type (wt) and the LXXXL mutant were expressed and proteolytically processed at comparable levels (Fig. 1B). Moreover, both wt GP and the LXXXL mutant were comparably incorporated into vesicular stomatitis virus (VSV) pseudoparticles (Fig. 1C), which are frequently used to study EBOV-GP-driven entry, and the LXXXL mutant mediated entry into cell lines derived from accidental hosts (humans and nonhuman primates) and a natural reservoir (fruit bat) with about half the efficiency of wt GP (Fig. 1D). Thus, the GXXXA motif is dispensable for GP expression and particle incorporation, and its mutation has only a modest effect on GP-driven host cell entry.

The integrity of GXXXA motif is essential for tetherin antagonism. Having demonstrated that the GXXXA motif is dispensable for GP expression and, to some 
A)

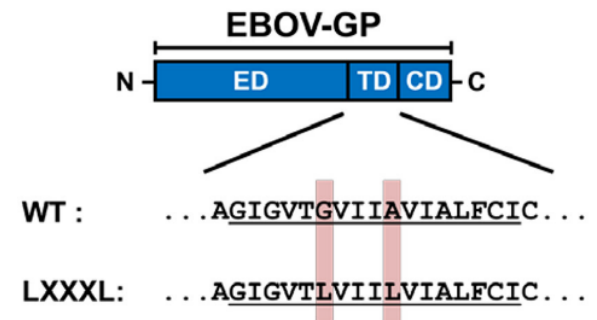

B)

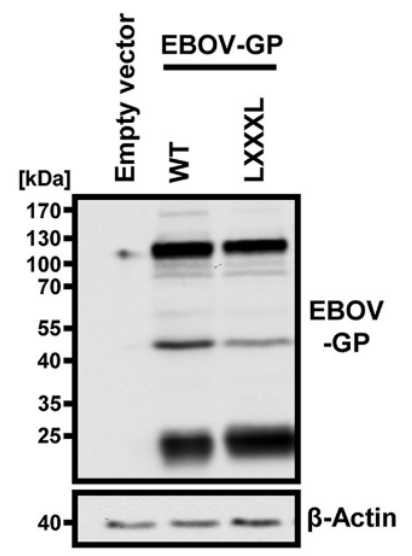

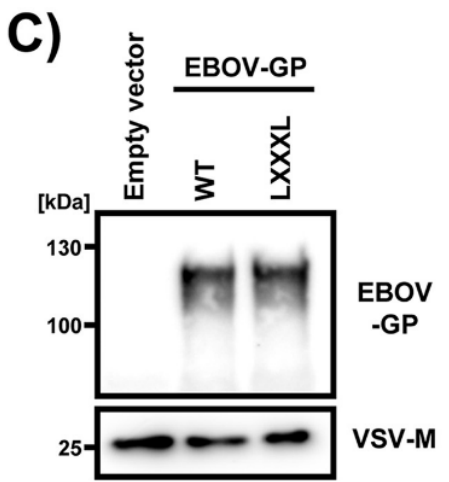

D)

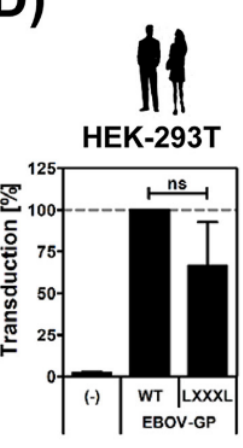

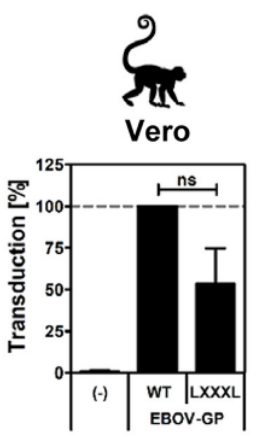

FIG 1 Mutation of a GXXXA motif in the EBOV-GP TMD is dispensable for GP expression and particle incorporation but slightly reduce GP-driven host cell entry. (A) Sequence of the GXXXA motif in the EBOV-GP wt and LXXXL mutant (ED, ectodomain; TD, transmembran domain [underlined]; $C D$, cytoplasmic domain). (B) Plasmids encoding V5-tagged versions of the indicated glycoproteins or empty plasmid (pCAGGS) as a negative control were transiently transfected into 293T cells. Expression of the GPs in cell lysates was detected using anti-V5 (1) (C) VSV pseudipes ho (he a 20\% sucrose cushion and then analyzed for the presence of EBOV-GP and VSV-M by Western blotting. The results were confirmed in three independent experiments. (D) The indicated human, nonhuman primate, and fruit bat cell lines were transduced with equal volumes of vesicular stomatitis virus (VSV) particles pseudotyped with the indicated GPs or with particles bearing no glycoprotein as negative controls. Luciferase activity in cell lysates, which was used as an indicator of transduction efficiency, was measured at $24 \mathrm{~h}$ postransduction. Shown are normalized data from four experiments performed with independent pseudotype preparations, in which transduction mediated by EBOV-GP wt was set as 100\%. Error bars indicate standard errors of the means, and statistical significance was analyzed using paired two-tailed $t$ tests (ns, not significant).

extent, for GP-driven host cell entry, we next investigated if the GXXXA motif is required for tetherin antagonism. For this endeavor, we first employed a previously documented virus-like particle (VLP) assay, in which release of VLPs is driven by the HIV-1 p55 Gag protein and is inhibited by tetherin (12). In the Gag-based assay, VLPs were readily released from tetherin-negative control cells, and release was markedly reduced upon expression of tetherin (Fig. $2 \mathrm{~A}$ and $\mathrm{B}$ ). The tetherin-mediated restriction of VLP release was rescued upon coexpression of HIV-1 Vpu and EBOV-GP wt (Fig. 2A and B), as expected. In contrast, the LXXXL mutant was largely unable to promote VLP release from tetherin-positive cells (Fig. 2A and B), and this defect could not be rescued by expressing large amounts of the mutant (data not shown). Thus, the GXXXA motif is essential for efficient tetherin counteraction, at least under the conditions studied.

We next studied whether the LXXXL motif is also required for rescue of the release of EBOV-like particles from blockade by tetherin. For this, the above-described VLP 
A) Gag
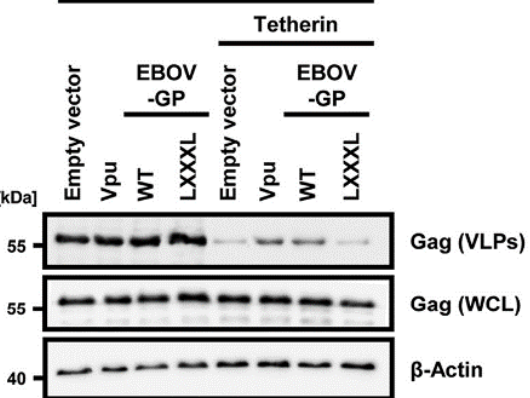

C) VP40

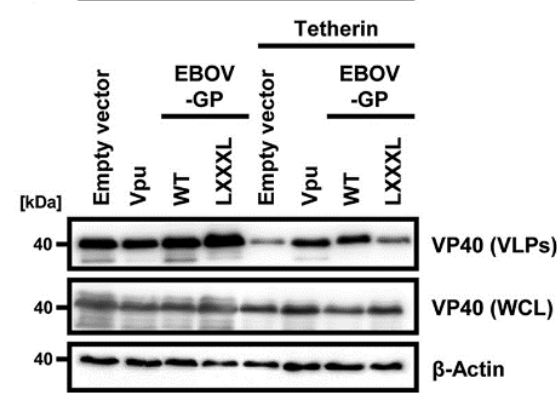

B)

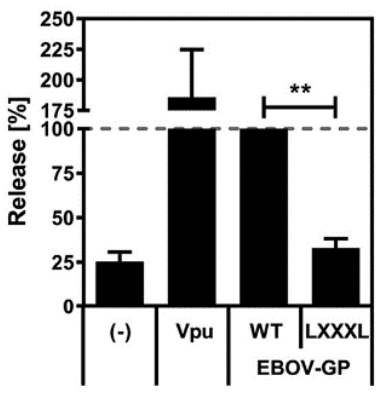

D)

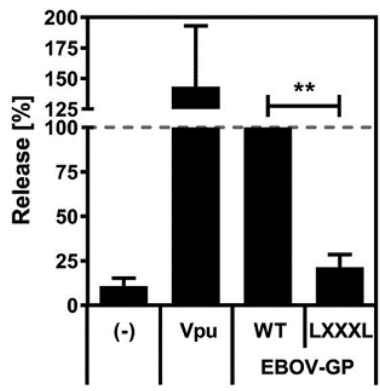

FIG 2 The GXXXA motif is required for tetherin antagonism. (A) 293T cells were cotransfected with plasmids encoding HIV-Gag, the indicated glycoproteins or Vpu, and tetherin or empty plasmid. Cells and supernatants were harvested at $48 \mathrm{~h}$ posttransfection. Virus-like particles (VLPs) were pelleted by centrifugation through a 20\% sucrose cushion. Whole-cell lysates (WCL) and VLPs were analyzed for the presence of Gag by Western bloting. Detection of $\beta$-at representative experiment are shown. (B) Three independent experiments conducted as descibed for panel A were quantified using the ImageJ program. VLP release from cells coexpressing EBOV-GP wt and tetherin was set as $100 \%$. Error bars indicate standard errors of the means, and statistical significance was analyzed using a paired two-tailed $t$ test $\left.{ }^{\star \star}, P \leq 0.01\right)$. (C) VLP release was examined as described for panel A, but EBOV-VP40 instead of HIV-Gag was used for particle production. (D) Four independent experiments conducted as described for panel C were quantified using the ImageJ program. VLP release from cells coexpressing EBOV-GP wt and tetherin was set as 100\%. Error bars indicate standard errors of the means, and a paired two-tailed $t$ test was used to determine statistical significance $(* *, P \leq 0.01)$.

assay was repeated using EBOV VP40 instead of HIV Gag. Expression of VP40 is sufficient for release of filamentous particles from cells $(18,19)$ and thus mimics release of EBOV from infected cells. In this assay, expression of EBOV-GP wt modestly increased the release of VLPs from tetherin-negative control cells (2-fold increase on average; $n=4$ ), in keeping with previous studies $(20,21)$, and rescued particle release from blockade by tetherin (Fig. $2 \mathrm{C}$ and D). Notably, the LXXXL mutant also promoted VLP release from tetherin-negative cells ( 1.5 -fold increase on average; $n=4)$ but failed to rescue particle release from blockade by tetherin (Fig. $2 C$ and D). These results show that the GXXXA motif is also required for tetherin antagonism in the context of EBOV-like particles and that GP-mediated augmentation of particle release and GP-driven tetherin antagonism are genetically separable processes.

The requirement of the GXXXA motif for tetherin antagonism is independent of membrane cholesterol levels. The directed expression of GP in cells can induce cellular detachment $(22,23)$. A recent study reported that the mutation of the GXXXA motif to LXXXL or depletion of cholesterol reduces GP-driven cell detachment (17). We confirmed that mutation of GXXXA to LXXXL reduces cell detachment (Fig. $3 \mathrm{~A}$ ) and asked whether addition of cholesterol confers efficient tetherin antagonism to the 
A)

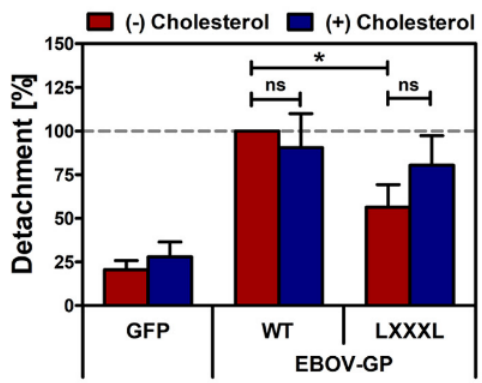

B)

Gag

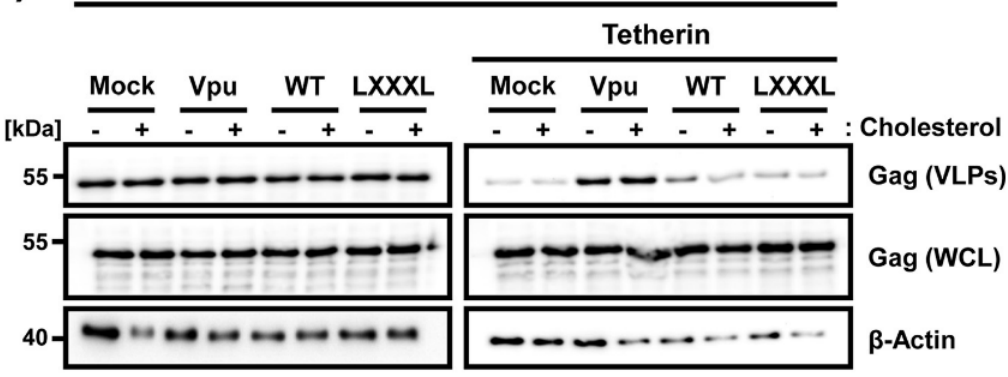

C)

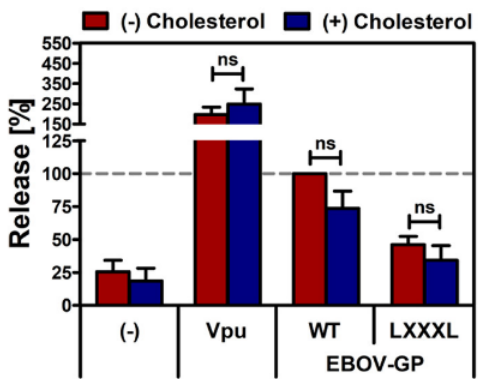

FIG 3 Defective tetherin antagonism by mutant LXXXL cannot by restored with cholesterol. (A) HeLa cells were transiently transfected with plasmids encoding the indicated glycoproteins or GFP as a negative control. At $6 \mathrm{~h}$ posttransfection, cells were treated with water-soluble cholesterol at a concentration of $100 \mu \mathrm{M}$ or water alone for $30 \mathrm{~min}$. Subsequently, cells were washed with PBS, and fresh medium was added. Supernatants were collected at $48 \mathrm{~h}$ after transfection, and the number of detached cells was quantified using flow cytometry. Shown are $48 \mathrm{~h}$ after transfection, and the number of detached cells was quantified using flow cytometry. Shown are normalized data from five independent experiments carried out with triplicates samples, for which detachment driven by EBOV-GP wt was set as 100\%. Error bars indicate standard errors of the means, and statistical significance performed as described in the legend of Fig. 2; but at $6 \mathrm{~h}$ posttransfection water-soluble cholesterol was added at a final concentration of $100 \mathrm{M}$ as indicated, and cells were incubated for $30 \mathrm{~min}$. Afterwards, the cells were washed and incubated overnight. The results of a single representative experiment are shown. (C) Thee inde pashed and inde pendent expeimis from cells coexpressing EBOV-GP wt and tetherin, but not treated with cholesterol, was set as $100 \%$. For panels $A$ and $C$, error bars indicate standard errors of the means, and statistical significance was analyzed using paired $t$ test (ns, not significant; ${ }^{*}, P \leq 0.05$ ).

LXXXL mutant. However, cholesterol did not endow the LXXXL mutant with efficient tetherin antagonism and even reduced tetherin counteraction by wt GP (Fig. 3B and C), although not to a statistically significant degree. Thus, the GXXXA motif in the TMD of EBOV-GP is essential for efficient tetherin antagonism, and the requirement for this motif for tetherin counteraction is independent of membrane cholesterol. 
The GXXXA motif is required for robust viral spread in tetherin-positive cells. Most previous analyses of tetherin counteraction by EBOV-GP were conducted with GP-transfected cells while infected cells were rarely studied. EBOV is a biosafety level 4 (BSL4) organism and could not be analyzed in the present study. However, VSV chimeras encoding EBOV-GP (VSV-EBOV-GP) afforded an opportunity to study EBOV-GP in the context of infected cells without the necessity of BSL4 conditions $(24,25)$. In order to analyze whether the GXXXA motif contributes to VSV-EBOV-GP spread in tetherin-positive cells, we generated VSV-EBOV-GP wt and VSV-EBOV-GP LXXXL via reverse genetics (Fig. $4 \mathrm{~A}$ ) and ensured by sequence analysis that the viruses had not acquired changes in GP during amplification in cell culture. Analysis of viral particles confirmed that the GP wt and LXXXL mutant were comparably incorporated into particles (Fig. 4B), as expected (Fig. 1C). Moreover, investigation of GP expression in infected cells showed that both wt and mutant GPs were expressed at similar levels (Fig. 4C). In order to analyze tetherin sensitivity of viruses bearing GP wt and mutant LXXXL, we infected Vero cells, which were stably transfected with empty vector ( $\mathrm{PQCXIP,} \mathrm{control)} \mathrm{or} \mathrm{a} \mathrm{tetherin-encoding} \mathrm{vector} \mathrm{and} \mathrm{expressed} \mathrm{high} \mathrm{levels} \mathrm{of} \mathrm{tetherin}$ (Fig. 4D). VSV wt was included as a control since this virus is highly tetherin sensitive $(26,27)$, and so far no VSV protein has been identified that antagonizes tetherin in infected cells. Infection of the Vero control cells (pQCXIP) revealed that VSV replicated more efficiently than VSV bearing EBOV-GP (Fig. 4E), as expected (24). Replication of VSV-EBOV-GP wt and mutant LXXXL was also readily detectable; but peak viral titers observed upon infection with the LXXXL mutant were about 10-fold reduced, and replication kinetics were delayed compared to the kinetics of infection with VSVEBOV-GP wt virus (Fig. 4E). The expression of tetherin in target cells had a stronger inhibitory effect on peak viral titers (Fig. 4F) of VSV than of VSV-EBOV-GP, suggesting that GP-mediated tetherin antagonism might be operative in infected cells. More importantly, tetherin expression had a clearly more profound inhibitory effect on spread of the VSV-EBOV-GP LXXXL mutant than the VSV-EBOV-GP wt virus (Fig. $4 \mathrm{E}$ and $F$ ), indicating that the GXXXA motif was required for tetherin counteraction in VSVEBOV-GP-infected cells.

\section{DISCUSSION}

It remains largely enigmatic how EBOV-GP counteracts tetherin. Recent studies sought to obtain insights via mutagenic analyses. They showed that the glycan cap, the $R B D$, and the TMD contribute to tetherin antagonism $(10,15,16)$. The observation regarding the TMD, jointly with the finding that a GXXXA motif within the EBOVGP transmembrane domain is required for GP-mediated cellular detachment (17), prompted us to investigate whether the GXXXA motif contributes to tetherin antagonism. We show that the motif is dispensable for GP expression and particle incorporation but is required for efficient GP-driven tetherin antagonism. Our results underline an important contribution of the TMD to tetherin counteraction and provide, for the first time, evidence that GP can inhibit tetherin when expressed in the context of cells infected with an EBOV surrogate.

Our previous work provided the first hints toward a role of the EBOV-GP TMD in tetherin counteraction. We showed that the GP2 subunit of GP interacts with tetherin (12) and that replacing the EBOV-GP TMD with that of the Lassa virus glycoprotein (LASV-GPC, which does not antagonize tetherin) abrogates tetherin antagonism (16). However, only limited conclusions could be drawn from these results since EBOV-GP harboring the LASV-GPC TMD was unable to mediate entry (16). Moreover, LASV-GPC equipped with the EBOV-GP TMD failed to counteract tetherin (16). Strong evidence for a role of the EBOV-GP TMD in tetherin counteraction was then provided by Vande Burgt and colleagues. They showed that soluble GP (sGP), which is the primary translation product of the GP open reading frame (ORF), fails to counteract tetherin and that addition of the EBOV-GP TMD to SGP is sufficient to bestow tetherin antagonism upon sGP (15). However, the TMD amino acids required for tetherin antagonism remained unknown. 
A)

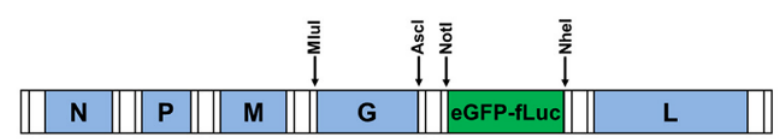

$\underbrace{}_{\substack{\text { Mlul } \\+ \\ \text { Ascl }}}$

\begin{tabular}{|c|c|c|c|c|c|}
\hline $\mathbf{N}$ & $\mathbf{P}$ & M & EBOV-GP & eGFP-fLuc & 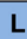 \\
\hline
\end{tabular}

B)

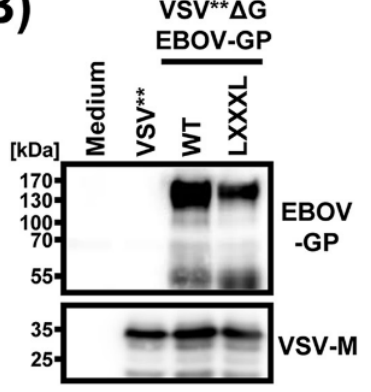

E)
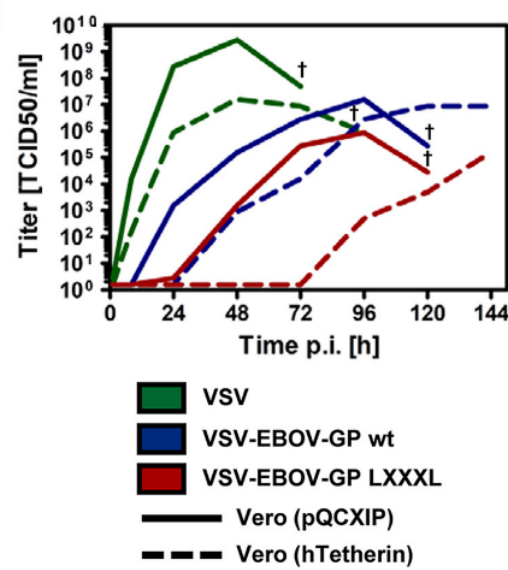

C) $\quad \operatorname{vSV}^{\star \star} \Delta G$

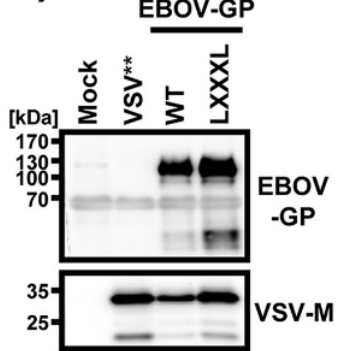

D)

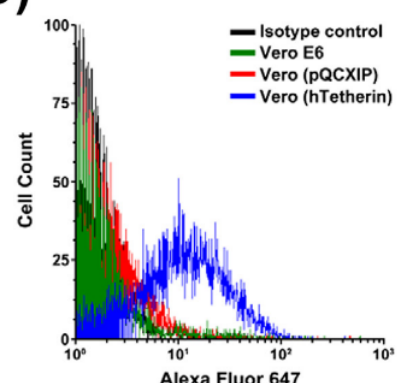

F)

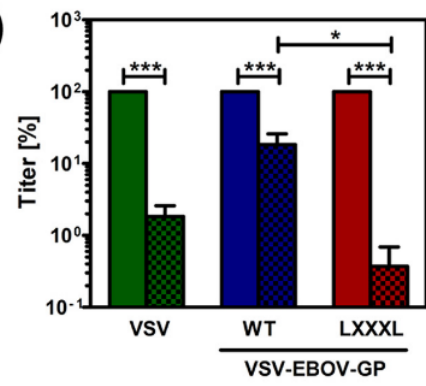

$\square$ Vero (pQCXIP)

Wero (hTetherin)

FIG 4 Disruption of the GXXXA motif reduces EBOV-GP-dependent viral spread in tetherin-positive cells. (A) Schematic illustration of the VSV** genome and the derived chimeric genome harboring the open reading frame (ORF) for EBOV-GP (red) instead of VSV-G. Transcription units coding for structural proteins of VSV are highlighted in light blue, while nontranslated regions of the genome are displayed in white. All VSV genomes code for a dual reporter consisting of eGFP linked to firefly luciferase from an additional transcription unit downstream of the respective glycoprotein ORF (green). (B) Replication-competent VSV chimeras encoding the indicated glycoproteins instead of VSV-G were pelleted through a 20\% sucrose cushion and evaluated for incorporation of EBOV-GP VSV wt or the indicated VSV chimeras at an MOI of 1 . At $20 \mathrm{~h}$ postinfection, cell lysates were prepared and analyzed for the expression of EBOV-GP ( $w$ t and mutant LXXXL) and VSV-M by Western blotting. Mock-infected and VSV wt-infected cells served as controls. Similar results were obtained in two independent experiments. (D) Tetherin surface expression on Vero cell lines used for the experiment shown in panel E was analyzed by flow cytometry. Vero (PQCXIP) were incubated with an isotype control and secondary antibody (black) or Vero E6 (green), Vero (pQCXIP) (red), Vero (hTetherin) (blue) cells were incubated with anti-tetherin antibody and secondary antibody. Antibody staining was analyzed by flow cytometry. A single representative experiment is shown; similar results were obtained in a second independent experiment (E) Control Vero cells transduced with plasmid POCXIP and Vero cells transduced to mutant ( $M$ Of 0.000 ) for 1 h $11,8,24,48,72,96,120$, alum taken at different time points $\left(1,8,24,48,72,96,120\right.$, and $144 \mathrm{~h}$ postinfection), and viral titers were quantified by $\operatorname{TCID}_{50}$ assay. The results of a single representative experiment are shown. Similar results were obtained in two additional, independent experiments. (Continued on next page) 
Our results show that a GXXXA motif within the TMD of EBOV-GP is essential for tetherin counteraction. The underlying mechanism is at present unclear. However, inferences can be made based on the work by Hacke and colleagues. They showed that the well-known ability of EBOV-GP to mediate the detachment of cells from a culture flask depends on the integrity of a GXXXA motif within the TMD and on the availability of membrane cholesterol (17). On the basis of these findings, they postulated that the GXXXA motif is required for interactions between GP trimers in cholesterol-rich microdomains (lipid rafts), which in turn is a prerequisite for GP-mediated detachment of cells and viral budding. Our results confirm that alteration of the GXXXA motif reduces cellular detachment and suggest that this defect can be rescued by cholesterol However, addition of cholesterol to cells expressing the LXXXL mutant did not restore tetherin antagonism, and a previous report suggested that GP is neither located in lipic rafts nor alters tetherin localization in such domains (14). Moreover, evidence was provided that targeting SGP to lipid rafts is not sufficient for tetherin antagonism (15) In the light of these findings, membrane cholesterol does not seem to play a major role in tetherin antagonism by GP, and alternative scenarios should be considered. For instance, the GXXXA motif might contribute to formation and/or stability of GP trimers, and reduced trimerization might be incompatible with tetherin antagonism. However our initial analyses revealed that particles bearing EBOV-GP wt and mutant LXXXL were comparably sensitive to inactivation by elevated temperature (data not shown), suggesting that the stability of the respective GP trimers may not be markedly different. Alternatively, the GXXXA motif might promote protein-protein interactions essential for tetherin antagonism. Given that the sequence integrity of the tetherin TMD is dispensable for GP-mediated tetherin antagonism (13), one can speculate that the GXXXA motif might mediate interactions of GP with a cellular protein that is required for tetherin counteraction.

Most studies employed VLP systems to analyze tetherin counteraction by EBOV-GP. In contrast, it remains unknown whether GP counteracts tetherin in the context of EBOV infection. Two studies provided evidence that EBOV infection might not be appreciably inhibited by tetherin $(12,28)$, but whether lack of tetherin sensitivity was due to the action of GP was not addressed. In the present study, alteration of the GXXXA motif in the context of VSV-EBOV-GP did not impact particle incorporation of GP and GP expression levels in infected cells. Moreover, mutation of the GXXXA motif only modestly reduced replication kinetics and peak viral loads in tetherin-negative cells, in keeping with the modestly diminished viral entry observed for the LXXXL mutant in single-cycle assays. In contrast, replication kinetics of the $\mathrm{XXXXL}$ mutant in tetherinpositive cells were markedly delayed compared to the kinetics of control cells while a clearly less pronounced delay was observed for the virus bearing wt GP. These results, jointly with the observation that VSV (which does not encode a tetherin antagonist that is active in infected cells) is more robustly inhibited by tetherin than VSV-EBOV-GP, suggest that tetherin counteraction by GP can occur in infected cells. Altering the GXXXA motif in the context of authentic EBOV might thus allow determination of the impact of GP-mediated tetherin antagonism on viral spread.

Based on these collective observations, we identified a mutation that abrogates tetherin antagonism with reasonable selectivity and consider it possible that even higher selectivity might be attainable by mutating only the glycine or the alanine residue within the GXXXA motif. Moreover, we provide, for the first time, evidence that GP can counteract tetherin in the context of cells infected with an EBOV surrogate Future studies are required to confirm our results with infectious EBOV and to further elucidate how the GXXXA motif contributes to tetherin counteraction.

FIG 4 Legend (Continued)

Crosses indicate time points at which kinetics were stopped due to complete virus-induced cell lysis. (F) Wean viral peak titers (VSV wit, $48 \mathrm{~h}$; VSV-EBOV chimeras, $96 \mathrm{~h}$ postinfection) resulting from inoculation of Vero control (pQCXIP) cells and Vero cells expressing hTetherin measured in three independent experiments conducted as described for panel E. Error bars indicate standard errors of the means, and statistical significance was analyzed using one-way ANOVA with Bonferroni posttest analysis $\left({ }^{*}, P \leq 0.05 ;{ }^{* * *}, P \leq 0.001\right)$. 


\section{MATERIALS AND METHODS}

Cell culture. HEK-293T (human, kidney), HeLa (human, cervix carcinoma), Vero and their subdoned derivate Vero E6 (both African green monkey, kidney). BHK-21(G43) (hamster, kidney), and EpoNi/22.1 (Buettikofer's epauletted fruit bat, kidney) cell lines were used as targets for transfection, transduction, infection, and detachment experiments. HEK-293T and HeLa cells (29) were obtained from DSMZ (ACC-635; Leibniz Institute DSMZ-German Collection of Microorganisms and Cell Cultures) and the NIH AIDS reagent program (https://Www.aidsreagent.org/), respectively, while Vero, Vero E6, BHK-21(G43) (30) and EpoNi/22.1 (12) cells were provided by collaborators. All cell lines were maintained in Dulbecco's modified Eagle medium (PAN-Biotech), supplemented with 10\% fetal bovine serum (Biochrom) and $1 \%$ penicillin-streptomycin (PAN-Biotech). Medium for EpoNi/22.1 cells was additionally supplemented with $1 \mathrm{mM}$ sodium pyruvate (PAA Laboratories) and $1 \times$ nonessential amino acids (from a $100 \times$ stock solution: PAN-Biotech). After being transfected with the retroviral PQCXIP vector, Vero cells stably expressing human tetherin [Vero (hTetherin)] and Vero control cells expressing pOCXIP [Vero (pQCXIP)] were selected and maintained using 10 and $0.5 \mu \mathrm{g} / \mathrm{ml}$ of puromycin (Cayman chemical) respectively. All cells were incubated in a humidified atmosphere at $37^{\circ} \mathrm{C}$ and $5 \% \mathrm{CO}_{2}$. For subcultivation and seeding, cells were detached by either resuspension in fresh oulture medium (HEK-293T cells) or by the use of trypsin-EDTA (PAN-Biotech).

Plasmids. Expression plasmids encoding the proteins used in this study were described previously, as follows: EBOV and VSV glycoproteins EBOV-GP (GenBank accession number AF086833.2) and VSV-G, respectively $(10,31)$; human tetherin (32); HIV-1 Vpu (12); HIV-1 p55-Gag $(12,16)$; and EBOV-VP40 harboring an $\mathrm{N}$-terminal c-Myc epitope, EQKLISEEDL (33). The GXXXA motif in the transmembrane of EBOV-GP was genetically modified to LXXXL by employing overlap extension PCR. Expression plasmids for the coding sequences of VSV nudeoprotein (VSV-N), phosphoprotein (VSV-P), and RNA-dependent RNA-polymerase (VSV-L) were generated by amplifying the respective open reading frames (ORF) from a plasmid-encoded VSV anti-genome (Indiana strain; kindly provided by G. Zimmer) and inserting them via standard cloning procedures into the pCAGGS expression vector.

In order to produce replication-competent, chimeric VSV expressing EBOV-GP wild-type (wt) or the LXXXL mutant instead of VSV-G, as well as a dual reporter consisting of an enhanced green fluorescent protein (eGFP) and firefly luciferase (fluc) from an additional transcription unit, we generated a plasmid-encoded VSV anti-genome (GenBank accession number J02428.1) as follows. First, we introduced unique Mlul and Nhel restriction sites upstream and downstream of the VSV-G transcription unit respectively. Next, a cassette was inserted that consisted of the coding sequences of VSV-G and the eGFP-fluc dual reporter, separated by a minimal intergenic region (34). To obtain the eGFP-fluc dual reporter, the stop codon of the eGFP open reading frame (ORF) was removed and then combined with the genetic information of fluc linked via a nonflexible amino add linker sequence (GPDPPVAD). This strategy gave rise to $\mathrm{VSV}^{\star \star}$ (the two asterisks indicate the dual reporter, later referred to as VSV). As this construct also contained a unique Ascl restriction site downstream of the VSV-G transcription unit, it allowed us to replace VSV-G with the EBOV-GP wt or mutant LXXXL, thus generating VSV*^ $\triangle G$ EBOV-GP wt, VSVEBOV wt, VSV*A $\triangle$ G EBOV-GP LXXXL, and VSV-EBOV LXXXL. The integrity of all PCR-amplified sequences was verified by automated sequence analysis.

Detachment assay. HeLa cells were transiently transfected following calcium-phosphate precipitation with plasmids encoding EBOV-GP wt, EBOV-GP LXXXL, or GFP as a control. At $6 \mathrm{~h}$ posttransfection. cells were treated with water-soluble cholesterol (Sigma) at a concentration of $100 \mu \mathrm{M}$ or water alone for $30 \mathrm{~min}$. Subsequently, cells were washed with phosphate-buffered saline (PBS), and fresh medium was added. Supernatants were collected at $48 \mathrm{~h}$ after transfection, and the number of detached cells was quantified using a Becton Dickinson LSR II flow cytometer and BD FACSDIVA software.

Production of rhabdoviral pseudotypes. The pseudotypes were generated and used for transduction as described previously (35). In brief, 293T cells were seeded in six-well plates and transfected by calcium phosphate precipitation with plasmids encoding VSV-G, EBOV-GP wt, or EBOV-GP LXXXL or with an empty plasmid (pCAGGS) as a negative control. At $18 \mathrm{~h}$ posttransfection, the cells were inoculated with a replication-deficient VSV, in which the ORF for VSV-G was replaced by two separate ORFs for eGFP and fluc (36) (kindly provided by G. Zimmer), at a multiplicity of infection (MOI) of 3. At $1 \mathrm{~h}$ postinfection, cells were washed with PBS and incubated with a 1:1,000 dilution of I1 (an anti-VSV-G mouse hybridoma supernatant from CRL-2700; American Type Culture Collection) for $1 \mathrm{~h}$ at $37^{\circ} \mathrm{C}$ in order to neutralize residual input virus. Finally, fresh culture medium was added to the cells. At 18 to $20 \mathrm{~h}$ postransduction supernatants were collected, darified from cell debris by centrifugation, aliquoted, and stored at $-80^{\circ} \mathrm{C}$ until use.

Transduction of cell lines and quantification of fluc activity. To assess cell entry driven by EBOV-GP LXXXL, 293T, Vero E6, and EpoNi/22.1 cells were seeded in 96 -well plates. At $24 \mathrm{~h}$ after seeding medium was removed, and cells were transduced with equal volumes of VSV pseudotypes. At 16 to 18 h postransduction, intracellular fluc activity was measured as an indicator of transduction efficiency. For this, the cell culture medium was aspirated, and cells were incubated for $30 \mathrm{~min}$ at room temperature with $50 \mu$ of luciferase cell culture lysis reagent (Promega). Lysates were transferred to a white opaque-walled 96-well plate (Thermo Scientific), and fluc activity was measured in a micooplate reade (Plate Chameleon; Hidex) using MicroWin2000 software (version 4.44; Mikrotek Laborsysteme, GmbH) and fluc substrate from a Beetle-Juice kit (PJK GmbH).

Rescue and quantification of replication-competent VSV chimeras encoding EBOV-GP wt and EBOV-GP LXXXL. For the rescue of the replication-competent VSV-EBOV wt or LXXXL, we used a strategy developed by others (37) with some modifications. First, BHK-21(G43) cells were seeded in 12-well plates and treated with mifepristone (Sigma-Aldrich) at a final concentration of $1 \mathrm{nM}$ [BHK-21(G43) cells 
inducibly expres5 VSV-G upon stimulation with mifepristonel (30). At $16 \mathrm{~h}$ postreatment, cells were infected with recombinant modified vaccinia virus Ankara expressing 77 polymerase (VMVA-T7; kindly provided by G. Sutter) (38) at an MOl of 3 . The inoculum was aspirated $1 \mathrm{~h}$ after infection, and cells were washed with PBS. Next, the cells were transfected with expression plasmids for VSV-N, $P$, and $-L$ (polymerase complex) as well as the respective plasmid-encoded VSV anti-genome using Lipofectamine 2000 (Thermofisher Scientific) as a transfection reagent, in this anti-genome, a 17 promoter sequence that precedes the leader region of the VSV genome drives cytoplasmic synthesis for negative-sense full-length VSV genomes with defined genome ends through the activity of the hepatitis delta virus ribozyme located directly downstream of the trailer region. The negative-sense, full-fength VSV genomes serve then as templates for mRNA transcription and further genome replication (both driven by the VSV polymerase complex). The following DNA amounts per well were used: $0.4 u g$ of VSV-N, $0.35 u g$ of VSV-P. $0.25 \mu \mathrm{g}$ of VSV $\mathrm{L}$, and $1 \mu \mathrm{g}$ of plasmid-encoded VSV anti-genome. At $6 \mathrm{~h}$ posttransfection, cells were washed; and fresh medium containing 1 niv mifepristone was added. We used mifepristone-stimulated BHK-2.1(G43) cells for initial transcomplementation of newly produced VSV particles with VSV-G in orde to boost rescue efficiency. At $18 \mathrm{~h}$ posttransfection, the culture medium was supplemented with 100 $\mu \mathrm{g} / \mathrm{ml}$ rifampin and $40 \mathrm{\mu g} / \mathrm{ml}$ cytosine $\beta-\mathrm{D}$-ar abinofuranoside (both from Sigma-Aldrich) to limit rMVA-T replication. After an additional $48 \mathrm{~h}$ of incubation, the supernatant was collected, clarified from cellular debris by centrifugation $\left(4,700 \mathrm{rpm}, 10 \mathrm{~min}, 4{ }^{\circ} \mathrm{O}\right)$, and twice filtered through $0.2-\mu \mathrm{m}$-pore-size filter membranes to exclude residual riMA-T7 from the preparation. Next; Vero E6 cells grown in a T-25 flask were inoculated with a $1: 3$ dilution of the filtered supernatant and incubated for $72 \mathrm{~h}$ in the presence of anti-VSV-G mouse hybridoma supernatant to neutralize subsequent host cell entry by in trons-delivered VSV-G. Afterwards, culture medium was collected and filtered again. Finally, Vero E6 cells grown in a T-75 flask were inoculated with a 1:10 dilution of the supernatant for virus stock production.

Quantification of titers of virus stocks was carried out on confluent Vero E6 cells seeded in 96-well plates. First, cell culture medium was removed and cells were inoculated with 10-fold serial dilutions of virus. At $1 \mathrm{~h}$ postinfection, inoculum was removed, and cell culture medium containing $2 \%$ methylcel lulose (Sigma-Aldrich) was added. After $48 \mathrm{~h}$ of incubation, eGFP-positive foci were counted under a fluorescence microscope. Virus stock titers were determined as the number of focus forming units (FFU) per millititer to calculate the MOI for subsequent infection experiments. To verify the integrity of VSV-EBOV wt and VSV-EBOV LXXXL, viral RNA was extracted from virus stocks using a QIAamp Viral RNA minikit (Oiagen) and reverse transcribed into CDNA using a SuperScript III first-strand synthesis system Thermofisher Scientific) according to the manufacturer's protocol (for random hexamers). A fragment of approximately 1,800 bp was then amplified with primers binding in the intergenic region upstream of EBOV-GP (forward) and near the $5^{\prime}$ end of the EGFP ORF (reverse) using Phusion polymerase (ThermoFisher Scientific), separated by agarose gel electrophoresis, and extracted from the gel by commercial kits (Macherey \& Nagel), before being subjected to automated sequence analysis (SeqLab)

Grow th kinetics of VSV-EBOV-GP in control and tetherin-positive cells. To evaluate the effect of the GXXXA motif on viral spread, Vero cells stably expressing human tetherin (hT etherin) or control Vero cells containing empty vector (pQCXIP) were infected with VSV-EBOV-GP wt or VSV-EBOV-GP LXXXL (MO of 0.0001$) \mathrm{for} 1 \mathrm{~h}$. Afterwards, cells were washed with PBS to remove residual inoculum, and fresh culture medium was added. Cells were further incubated, and samples were collected at $1,8,24,48,72,96,120$ and $144 \mathrm{~h}$ postinfection. Viral titers were determined on Vero $\mathrm{E} 6$ cells by caloulating the $50 \%$ tissue culture infective dose $\left(T C D_{50}\right)$ according to the Spearman-Kärber method $(39,40)$.

Analysis of tetherin surface expression by now cytometry. For analysis of tetherin surface expression, Vero E6. Vero control (pOOXIP), and Vero cells stably expressing human tetherin were detached and incubated for $30 \mathrm{~min}$ at $4^{\circ} \mathrm{C}$ with a 150 dilution of purifed anti-human (D317 (BST2 tetherin) antibody (Biolegend) or with an isotype-matched control antibody (Biolegend). Afterwards cells were washed twice with PBS and then incubated with Alexa Fluor 647-conjugated goat anti-mouse secondary antibody at a 1:100 dilution for $30 \mathrm{~min}$ at $4^{\circ} \mathrm{C}$. Finally, cells were washed with PBS, fixed with $2 \%$ paraformaldehyde (PFA); and analyzed in a Becton Dickinson LSF II flow cytometer. Data were further analyzed using FCS Express, version 4, software.

Immunoblotting. Expression and incorporation of EBOV-GP wt and EBOV-GP LXXXL into VSV pseudotypes and replication-competent VSV-chimeras were determined by Western blotting. For ex pression analysis, HEK-293T cells were transiently transfected with plasmids encoding EBOV-GP wh EBOV-GP LXXXL (both harboring an N-terminal V5 tag), or empty plasmid (pCAGGS) as a negative control. At $48 \mathrm{~h}$ posttransfection, culture medium was aspirated, and cells were lysed using $300 \mu \mathrm{l}$ of $2 \times$ sodium dodecyl sulfate (SDS)-containing lysis buffer $(50 \mathrm{mM}$ Tris [pH $6.81,10 \%$ glycerol. $2 \%$ SDS, 5\% $\beta$-mercaptoethanol, $0.1 \%$ bromophenol blue, $1 \mathrm{miM}$ EDTA) and boiled for $30 \mathrm{~min}$ at $95^{\circ} \mathrm{C}$ To assess EBOV-GP wt and EBOV-GP LXXXL. incorporation into pseudotypes and VSV-EBOV chimeras, equal volumes of pseudotype preparations or supernatant of virus stocks were pelleted by centrifugation $\left(17,000 \times a, 2 \mathrm{~h}, 4^{\circ} \mathrm{O}\right)$ through a $20 \%$ sucrose ashion. Samples were resuspended in $20 \mathrm{w}$ of $2 \times$ SDS lysis buffer and boiled for $30 \mathrm{~min}$ at $95^{\circ} \mathrm{C}$. Afterwards, samples were separated by SDS-PAGE using $12.5 \%$ polyacrylamide gels and transferred onto nitrocellulose membranes $(0.2-\mu \mathrm{m}$ pore size; GE Healthcare Life Sciences). Membranes were blocked for $1 \mathrm{~h}$ in $5 \%$ milk powder in PBS with $0.1 \%$ Tween 20 . The expression of V5-tagged glycoproteins was detected using anti-V5 antibody (Invitrogen) at a 1:1,000 dilution: $\beta$-actin was detected after the membranes were stripped (Tris-HCL, SDS, $\beta$-mercaptoethanol $50^{\circ} \mathrm{C}, 30 \mathrm{~min}$ ) using an anti- 3 -actin antibody (Sigma) at a dilution of $1: 1,000$. Particle incorporation of EBOV-GPS was detected using a GP1-specific rabbit serum at a 1:1,000 dilution. To show that equal amounts of pseudotypes and replication-competent virus were used, a separate membrane with the same samples was incubated with an antibody against VSV-M (matrix protein, raised in mice KKeraFast] 
1:1,000). Bound antibodies were detected using horseradish peroxidase (HRP)-linked anti-mouse or anti-rabbit secondary antibodies (Dianova) at a dilution of 1:10,000. Signals of bound secondary antibodies were detected using an in-house-made enhanced chemiluminescence $(E C L)$ solution $(0.1 \mathrm{M}$ Tris- $\mathrm{HCl}_{,} \mathrm{pH}$ 8.6.250 $\mu \mathrm{g} / \mathrm{ml}$ luminol [Sigmal, $1 \mathrm{mg} / \mathrm{ml}$ para-hydroxycoum aric acid [Sigmal, $0.3 \% \mathrm{H}_{2} \mathrm{O}_{2}$ ) and visualized using a ChemoCam imaging system along with the ChemoStar Professional software (Intas).

Virus-like particle release assay. Release of HIV-Gag or EBOV-VP40-based virus-like particles (MPS) was analyzed as described before (10) to assess the importance of the GXXXA motif for EBOV-GPdependent tetherin antagonism. In brief, 293T cells were seeded in 48-well plates and cotransfected following calcium-phosphate precipitation with plasmid encoding HIV-1 p55-Gag or EBOV-VP40, human tetherin and a tetherin antagonist or empty plasmid as control. At $48 \mathrm{~h}$ posttransfection, supernatants were collected and clarified from cell debris, and VLPs were pelleted by centrifugation through a $20 \%$ sucrose cushion. Concentrated VLPs were resuspended in $20 \mu \mathrm{l}$ of $2 \times$ SDS buffer. In parallel, cells were lysed with $50 \mu \mathrm{l}$ of $2 \times$ SDS lysis buffer. Both whole-cell lysates (WCL) and VLPs were boiled at $95^{\circ} \mathrm{C}$ for $30 \mathrm{~min}$. Presence of HIV-Gag in lysates and VLPs was analyzed by immunoblotting according to the same protocol as described in the previous paragraph ("Immunoblotting"). Gag protein was detected using a 1:100 diluted supernatant of hybridoma cells secreting a mouse anti-Gag antibody (183-H12-5C) (41), while EBOV-VP40 was detected using undiluted supernatants of $9 E 10$ cells, a hybridoma cell line that secretes anti-Myc antibody (42). Finally, antibody binding was detected employing an HRP-linked anti-mouse secondary antibody (Dianova) at a dilution of 1:10,000.

Statistical analyses. To assess statistical significance, unpaired and paired Student $t$ tests were conducted for comparison of two data groups, while one-way analysis of variance (ANOVA) with Bonferroni posttest analysis was conducted for comparison of multiple data groups. All statistical analyses were done employing the GraphPad Prism software (GraphPad Software).

\section{ACKNOWLEDGMENTS}

The following reagents were obtained through the NIH AIDS Reagent Program, Division of AIDS, NIAID, NIH: the HeLa cell line from Richard Axel and anti-HIV-1 p24 hybridoma (183-H12-5C) from Bruce Chesebro. We thank G. Herrler for BHK-21(G43) cells and M. Müller and C. Drosten for EpoNi/22.1 cells.

The work of Michael Schindler (SCHI1073/4-1) and Stefan Pöhlmann (PO 716/8-1) was funded by Deutsche Forschungsgemeinschaft (DFG). Mariana González-Hernández was funded by the Deutscher Akademischer Austauschdienst (DAAD) country-related cooperation program with Mexico (CONACYT, Consejo Nacional de Ciencia y Tecnología) (stipend).

\section{REFERENCES}

1. Chow J, Franz KM, Kagan JC. 2015. PRRs are watching you: Localization of innate sensing and signaling regulators. Virology 479-480:104-109. https://doi.org/10.1016/j,virol.2015.02.051.

2. Schneider WM, Chevillotte MD, Rice CM. 2014. Interferon-stimulated genes: a complex web of host defenses. Annu Rev Immunol 32:513-545. https://doi.org/10.1146/annurev-immunol-032713-120231.

3. Schoggins JW, Wilson SJ, Panis M, Murphy MY, Jones CT, Bieniasz P, Rice CM. 2011. A diverse range of gene products are effectors of the type I interferon antiviral response. Nature 472:481-485. https://doi.org/10 .1038 /nature09907

4. Thompson MR, Kaminski JJ, Kurt-Jones EA, Fitzgerald KA. 2011. Pattern recognition receptors and the innate immune response to viral infection. Viruses 3:920-940. https://doi.org/10.3390/v3060920.

5. Erikson E, Adam T, Schmidt S, Lehmann-Koch J, Over B, Goffinet $C_{c}$ Harter $C_{t}$ Bekeredjian-Ding L, Sertel S, Lasitschka F, Keppler OT, 2011. In vivo expres. sion profile of the antiviral restriction factor and tumor-targeting antigen CD317/BST-2/HM1.24/tetherin in humans. Proc Natl Acad Sci U S A 108: 13688-13693. https://doi.org/10.1073/pnas.1101684108.

6. Neil SJ, Zang T, Bieniasz PD. 2008. Tetherin inhibits retrovirus release and is antagonized by HIV-1 Vpu. Nature 451:425-430. https://doi.org/10 .1038 /nature 06553 .

7. Perez-Caballero D, Zang T, Ebrahimi A, MANatt MW, Gregory DA, Johnson MC Bieniasz PD. 2009. Tetherin inhibits HIV-1 release by directly tethering virions to cells. Cell 139:499-511. https://doi.org/10.1016/j.cell .2009.08.039.

8. Neil SJ. 2013. The antiviral activities of tetherin. Curr Top Microbiol Immunol 371:67-104. https://doi.org/10.1007/978-3-642-37765-5_3

9. Messaoudi I, Amarasinghe GK, Basler CF. 2015. Filovirus pathogenesis and immune evasion: insights from Ebola virus and Marburg virus. Nat Rev Microbiol 13:663-676. https://doi.org/10.1038/nrmicro3524.

10. Brinkmann C Nehlmeier I, Walendy-Gnirss $\mathrm{K}$, Nehls J, Gonzalez HM,

July 2018 Volume 92 Issue 13 e00403-18
Hoffmann M, Qiu X, Takada A, Schindler M, Pöhlmann S. 2016. The tetherin antagonism of the Ebola virus glycoprotein requires an intact receptor-binding domain and can be blocked by GP1-specific antibodies. J Virol 90:11075-11086. https://doi.org/10.1128/JVI.01563-16.

11. Kaletsky RL, Francica JR, Agrawal-Gamse Cr Bates P. 2009. Tetherinmediated restriction of filovirus budding is antagonized by the Ebola glycoprotein. Proc Natl Acad Sa U S A 106:2886-2891. https://doi.org/ 10.1073/pnas 0811014106

12. Kühl A, Banning C, Marzi A, Votteler J, Steffen I, Bertram S, Glowacka I, Konrad A, Sturzl M, Guo JT, Schubert U, Feldmann H, Behrens G, Schindler M, Pöhlmann S. 2011. The Ebola virus glycoprotein and HIV-1 Vpu employ different strategies to counteract the antiviral factor tetherin. J Infect Dis 204(Suppl 3):S850-\$860. https://doi.org/10.1093/infdis/jir378.

13. Lopez LA, Yang $S J_{\text {, Hauser }} \mathrm{H}$, Exline $\mathrm{CM}_{\text {, }}$ Haworth $K \mathrm{G}_{\text {, Oldenburg J, }}$ Cannon PM. 2010. Ebola virus glycoprotein counteracts BST-2/tetherin restriction in a sequence-independent manner that does not require tetherin surface removal. J Virol 84:7243-7255. https://doi.org/10.1128/ JV.02636-09.

14. Lopez LA, Yang SJ, Exline CM, Rengarajan S, Haworth KG, Cannon PM. 2012. Anti-tetherin activities of HIV-1 Vpu and Ebola virus glycoprotein do not involve removal of tetherin from lipid rafts. J Virol 86:5467-5480. https://doi.org/10.1128/JM.06280-11.

15. Vande Burgt NH, Kaletsky RL, Bates P. 2015. Requirements within the Ebola viral glycoprotein for tetherin antagonism. Viruses 7:5587-5602. https://doi.org/10.3390/v7102888.

16. Gnirß K, Fiedler $M_{t}$ Kramer-Kuhl $A$, Bolduan $S$, Mittler $E_{c}$ Becker $S$, Schindler M, Pöhlmann S. 2014. Analysis of determinants in filovirus glycoproteins required for tetherin antagonism. Viruses 6:1654-1671. https://doi.org/10.3390/v6041654.

17. Hacke $M$, Bjorkholm $P$, Hellwig A, Himmels $P$, Ruiz de Almodovar $C$ Brugger B, Wieland F, Ernst AM. 2015. Inhibition of Ebola virus

jvi.asm.org 11 
glycoprotein-mediated cytotoxicity by targeting its transmembrane domain and cholesterol. Nat Commun 6:7688. https://doi.org/10.1038/ ncomms 8688

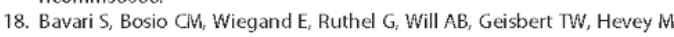
Schmaljohn C, Schmaljohn A, Aman MJ. 2002. Lipid raft microdomain a gateway for compartmentalized trafficking of Ebola and Marburg viruses. J Exp Med 195:593-602. https://doi.org/10.1084/jem.20011500.

19. Noda T, Sagara H, Suzuki Es Takada As Kida H, Kawaoka Y. 2002. Ebola virus VP40 drives the formation of virus-like filamentous particles along with GP. J Virol 76:4855-4865. https://doi.org/10.1128/JVl.76.10.4855 -4865.2002 .

20. Kühl A, Hoffmann M, Muller MA, Munster VJ, Gnirss K, Kiene $M$, Tsegaye TS, Behrens $G$, Herrler G, Feldmann H, Drosten C. Pöhlmann S. 2011 Comparative analysis of Ebola virus glycoprotein interactions with human and bat cells. J Infect Dis 204(Suppl 3):S840-S849. https://doi.org/ $10.1093 / \mathrm{infdis} / \mathrm{jir} 306$.

21. Licata JM, Johnson RF, Han Z Harty RN. 2004. Contribution of Ebola virus glycoprotein, nucleoprotein, and VP24 to budding of VP40 virus-like partides. J Virol 78:7344-7351. https://doi.org/10.1128/JVI.78.14.7344 -7351.2004 .

22. Simmons G, Wool-Lewis RJ, Baribaud F, Netter RC Bates P. 2002. Ebola virus glycoproteins induce global surface protein down-modulation and loss of cell adherence. J Virol 76:2518-2528. https://doi.org/10.1128/jvi .76.5.2518-2528.2002.

23. Yang ZY, Duckers HJ, Sullivan NJ, Sanchez A, Nabel EG, Nabel GJ. 2000. Identification of the Ebola virus glycoprotein as the main viral determinant of vascular cell cytotoxicity and injury. Nat Med 6:886-889. https:// doi.org/10.1038/78645.

24. Garbutt $M$, Liebscher $R$, Wahl-Jensen $V$, Jones $S$, Moller $P$, Wagner $R$, Volchkov V, Klenk HD, Feldmann $\mathrm{H}$, Stroher U. 2004. Properties of replication-competent vesicular stomatitis virus vectors expressing glycoproteins of filoviruses and arenaviruses. J Virol 78:5458-5465. https:// doi.org/10.1128/JVl.78.10.5458-5465.2004

25. Takada A, Feldmann $\mathrm{H}$, Stroeher U, Bray M, Watanabe $\mathrm{S}$, Ito $\mathrm{H}_{t}$ McGregor M, Kawaoka Y. 2003. Identification of protective epitopes on ebola virus glycoprotein at the single amino acid level by using recombinant vesicular stomatitis viruses. J Virol 77:1069-1074. https://doi.org/10.1128/JM $.77 .2 .1069-1074.2003$

26. Liberatore RA, Mastrocola EJ, Powell C Bieniasz PD, 2017. Tetherin inhibits cell-free virus dissemination and retards murine leukemia virus pathogenesis. J Virol 91:e02286-16. https://doi.org/10.1128/JVl .02286-16.

27. Weidner $\mathrm{JM}_{t}$ Jiang $\mathrm{D}$, Pan $\mathrm{XB}$, Chang J, Block TM, Guo JT. 2010. Interferon-induced cell membrane proteins, IFITM3 and tetherin, inhibit vesicular stomatitis virus infection via distinct mechanisms. J Virol 84: 12646-12657. https://doi.org/10.1128/JVI.01328-10.

28. Radoshizky SR, Dong L, Chi X, Clester JC, Retterer C, Spurgers K, Kuhn $\mathrm{JH}$, Sandwick S, Ruthel G, Kota K, Boltz D, Warren T, Kranzusch PJ, Whelan SP, Bavari S. 2010. Infectious Lassa virus, but not filoviruses, is restricted by BST-2/tetherin. J Virol 84:10569-10580. https://doi.org/10.1128/JVI $.00103-10$.

29. Maddon PJ, Dalgleish AG, McDougal JS, Clapham PR, Weiss RA, Axel R. 1986. The T4 gene encodes the AIDS virus receptor and is expressed in the immune system and the brain. Cell 47:333-348. https://doi.org/10 $1016 / 0092-8674(86) 90590-8$
30. Hanika A, Larisch B, Steinmann E, Schwegmann-Wessels C, Herrler G, Zimmer G. 2005. Use of influenza C virus glycoprotein HEF for generation of vesicular stomatitis virus pseudotypes. J Gen Virol 86: 1455-1465. https://doi.org/10.1099/vir.0.80788-0.

31. Hoffmann M, Grone L, Dietzel E, Paijo J, Gonzalez-Hernandez $M$ Nehlmeier I, Kalinke U, Becker S, Pöhlmann S. 2017. A polymorphism within the internal fusion loop of the Ebola virus glycoprotein modulates host cell Entry. J Virol 91:e00177-17. https://doi.org/10.1128/ JVI.00177-17.

32. Sauter D, Schindler M, Specht A, Landford WN, Munch J, Kim KA, Votteler J, Schubert U, Bibollet-Ruche F, Keele BF, Takehisa J, Ogando Y, Ochsenbauer C, Kappes JC, Ayouba A, Peeters M, Learn GH, Shaw G, Sharp PM Bieniasz P, Hahn BH, Hatziioannou T, Kirchhoff F. 2009. Tetherin-driven adaptation of $\mathrm{Vpu}$ and Nef function and the evolution of pandemic and nonpandemic HIV-1 strains. Cell Host Microbe 6:409-421. https://doi .org/10.1016/j.chom.2009.10.004.

33. Martin-Serrano J, Zang T, Bieniasz PD. 2001. HIV-1 and Ebola virus encode small peptide motifs that recruit Tsg101 to sites of partide assembly to facilitate egress. Nat Med 7:1313-1319. https://doi.org/10 $.1038 / \mathrm{nm} 1201-1313$

34. Schnell MJ, Buonocore L, Whitt MA, Rose JK. 1996. The minimal conserved transcription stop-start signal promotes stable expression of a foreign gene in vesicular stomatitis virus. J Virol 70:2318-2323.

35. Hoffmann M, Gonzalez HM, Berger E, Marzi A, Pöhlmann S. 2016. The glycoproteins of all filovirus species use the same host factors for entry into bat and human cells but entry efficiency is species dependent. PLoS One 11:e0149651. https://doi.org/10.1371/journal.pone. 0149651.

36. Berger Rentsch M, Zimmer G. 2011. A vesicular stom atitis virus repliconbased bioassay for the rapid and sensitive determination of multispecies type I interferon. PLoS One 6:e25858. https://doi.org/10.1371/ journal.pone.0025858.

37. Lawson ND, Stillman EA, Whitt MA, Rose JK. 1995. Recombinant vesicular stomatitis viruses from DNA. Proc Natl Acad Sci U S A 92:4477-4481.

38. Sutter $G$, Ohlmann $M$, Erfle V. 1995. Non-replicating vaccinia vector efficiently expresses bacteriophage T7 RNA polymerase. FEBS Lett 371: 9-12. https://doi.org/10.1016/0014-5793(95)00843-X

39. Dietzel $E$, Kolesnikova L, Sawatsky B, Heiner A, Weis M, Kobinger GP Becker S, M von V, Maisner A. 2015. Nipah virus matrix protein influences fusogenicity and is essential for particle infectivity and stability. J Viro 90:2514-2522. https://doi.org/10.1128/JVl.02920-15.

40. Krähling V Becker D, Rohde C Eickmann M. Eroglu Y Herwig A Kerber R, Kowalski K. Vergara-Alert J, Becker S. 2016. Development of an antibody capture ELISA using inactivated Ebola Zaire Makona virus. Med Microbiol Immunol 205:173-183. https://doi.org/10.1007/s00430-015 -0438-6.

41. Chesebro B, Wehrly K, Nishio J, Perryman S. 1992. Macophage-tropic human immunodeficiency virus isolates from different patients exhibit unusual V3 envelope sequence homogeneity in comparison with T-cell tropic isolates: definition of critical amino acids involved in cell tropism. tropic isolates: definition
$\mathrm{J}$ Virol 66:6547-6554.

42. Evan Gl, Lewis GK, Ramsay G, Bishop JM. 1985. Isolation of monodonal antibodies specific for hum an c-myc proto-oncogene product. Mol Cell Biol 5:3610-3616. https://doi.org/10.1128/MCB.5.12.3610. 


\section{Discussion}

Ebola virus constitutes a severe threat to human health. Understanding how EBOV enters cells and evades control by the immune system may pave the way to novel options for antiviral intervention.

EBOV must be handled in biosecurity level 4 (BSL-4) laboratories which slows down research progress. Therefore, surrogate systems have been developed to study EBOV infection. For instance, MLV- and VSV-vectors pseudotyped with EBOV-GP (MLVpp, VSVpp) have been used to study viral entry [248-250]. Moreover, EBOV-like particles containing VP30 linked to luciferase (VP30-luc) are used to study viral entry in the context of exclusively EBOV-derived proteins [128]. However, VP30-Luc EBOV-VLPs, VSVpp and MLVpp allow only one round of infection and are not able to replicate. In contrast, a novel transcription- and replication-competent virus-like particle (trVLP) minigenome system allows to study all steps of EBOV infection [417] in the context of authentic filamentous particles [418].

Similar to other viral glycoproteins, including influenza hemagglutinin [419], EBOV-GP depends on priming by host cell proteases to transit into a membrane-fusion competent form. Proteolytic priming of EBOV-GP can be facilitated by the endosomal cysteine proteases CatB/CatL [241,248]. However, CatB/CatL dependence was mainly documented with surrogate systems and a study conducted with authentic EBOV and CatB or CatL knockout mice found that expression of these proteases was dispensable for viral spread and pathogenesis [251]. Therefore, the first goal of this work was to identify determinants governing the CatB/L dependence of EBOV-GP-driven entry into host cells. The results of this research are reported in manuscript 1 and will be discussed below.

EBOV-GP post-translational modifications involve cleavage by furin into the subunits GP1 and GP2. However, this cleavage is dispensable for GP-driven entry [420-422]. In contrast, removal of the MLD and glycan cap in host cell endosomes exposes the RBD and is required for viral entry. These cleavage events are facilitated by CatB/CatL [241]. CatB/CatL are involved in apoptosis and have been targeted for treatment of diseases like traumatic brain injury [423], cancer [424] or viral infections [425]. Their 
enzymatic activity can be inhibited by E-64d, an epoxysuccinate inhibitor, and MDL28170, an aldehyde inhibitor [426-429], and both compounds inhibit EBOV-GP-driven entry [243,248,250]. In the present study, these inhibitors and the above listed surrogate systems were used to determine whether the shape of the GP presenting particle impacts CatB/CatL dependence of GP-driven entry. These studies were motivated by the observation that many published studies examining CatB/CatL dependence of GP-driven entry used spherical retroviral or bullet shaped rhabdoviral particles [220,241,247,248,250], which might be taken up into cells via different mechanisms [220,221] as compared to filamentous particles $[84,182,223]$ and might thus exhibit differential CatB/CatL dependence. I was able to show that GP-driven entry was CatB/CatL dependent irrespective of whether GP was presented in the context of spherical, bullet shaped or filamentous particles and irrespective of the target cell line.

My results suggested that host cell entry of filamentous particles bearing GP is CatB/CatL dependent and hence fails to provide an explanation for the striking finding that CatB or CatL are dispensable for EBOV spread in mice [251]. A second scenario that may account for the findings of Marzi and colleague is that other cathepsins can compensate for the loss of CatB or CatL expression. These compensatory enzymes might be responsible for GP processing and allow efficient spread in mice. In this context, it is important to note that although E-64d and MDL28170 inhibit CatB and CatL respectively, they can also interfere with the proteolytic activity of other cysteine proteases and may thus block the activity of compensatory proteases.

My studies revealed that the lung derived cell line Calu-3 is largely refractory to entry driven by diverse filovirus glycoproteins. In contrast, the cells were susceptible to entry driven by rhabdo-, paramyxo- and arenavirus glycoproteins. The absence of robust GP-driven entry was striking, since EBOV has a broad cell tropism and its GP can drive entry into a diverse range of cells, with only $T$ and $B$ cells being refractory for incompletely understood reasons [249,430-432]. A study by Park and colleagues revealed that Calu-3 cells express low levels of CatL. Therefore, uncleaved Middle East respiratory syndrome coronavirus (MERS-CoV) spike protein is unable to mediate entry into Calu-3 cells [433]. This finding raised the question whether the low 
expression levels of CatL could also account for the inefficient EBOV-GP-driven entry into Calu-3 cells. Using quantitative RT-PCR, I could confirm that Calu-3 cells express lower mRNA levels of CatL than 293T or Huh-7 cell lines, while CatB mRNA copies were only slightly lower than those measured for 293T and Huh-7 cells. In order to reveal if CatL expression limits filovirus GP-driven entry, I generated Calu-3 cell lines that stably express CatL. I could show that directed expression of CatL significantly increased MARV-GP-driven entry and moderately improved EBOV-GP-, SUDV-GP-, TAFV-GP-, RESTV-GP-driven entry but did not appreciably enhance BDBV- and LLOV-GP-driven entry and had no effect on VSV-G driven entry which is CatL independent. My observations are in keeping with previous studies which show that CatB/CatL dependence varies among filovirus GPs [247,250]. For instance, directed expression of CatB but not CatL in CatB/CatL knockout cells promotes EBOV-GP- and TAFV-GP-driven entry. In contrast, CatL but not CatB increased MARV-GP-driven entry while expression of both enzymes promoted RESTV-GP-driven entry [247].

Expression of attachment factors can greatly augment EBOV-GP-driven entry. Therefore, in parallel to CatL, I also evaluated whether filovirus attachment factors (DC-SIGN, AxI) or receptor (NPC1) could account for limited GP-driven entry into Calu-3 cells. To investigate this, I generated stable Calu-3 cell lines expressing either DC-SIGN, AxI, NPC1 or CatB. I could show that CatB, NPC1 and AxI do not increase entry into Calu-3 cells. The finding that directed expression of CatB and NPC1 did not rescue was not unexpected since it is well known that these proteins are ubiquitously expressed [229,252,253]. In contrast, expression of Axl is cell line dependent. However, its entry enhancing activity should not depend on the cellular context and it is unclear why directed expression of Axl failed to augment entry into Calu-3 cells. One can speculate that expression levels of Axl in Calu-3 cells might have been insufficient for entry augmentation. In contrast, directed expression of DC-SIGN which can boost GP-driven entry up to 50-fold [200,216] and renders Jurkat cells susceptible to GP-driven entry [216], rescued EBOV-GP, SUDV-GP and MARV-GP entry into Calu-3 cells. Moreover, DC-SIGN expression moderately increased entry driven by RESTV-GP and TAFV-GP. Finally, I provided evidence that increased entry into Calu-3 cells engineered to express DC-SIGN or CatL was due to direct effects of these 
proteins on the entry reaction. In sum, my results indicate that entry driven by filovirus GPs in Calu-3 cells is limited at the stage of attachment and priming.

The IFN system is the first line of defense against viral infections. Upon infection, signaling cascades stimulate IFN production, and IFN then triggers the expression of approximately 500 ISGs, many of which exert antiviral activity $[300,301,306]$. Tetherin is an IFN-induced protein that prevents viral release by forming a physical bridge between viral and cellular membrane [347,359,360]. Several viruses encode proteins that antagonize tetherin, usually by interfering with tetherin expression $[361,383]$. Filovirus GPs can antagonize tetherin but the mechanism used by these proteins to counteract tetherin remains incompletely understood. Therefore, the second goal of my research was to identify domains and amino acid motifs in GP that are required for tetherin antagonism. The results of my research have been documented in manuscript 2 and will be discussed below.

It has been shown that the glycan cap, RBD and TMD in GP contribute to tetherin antagonism [413-415]. However, no specific amino acids within the TMD have been identified that are required for tetherin antagonism. However, a GXXXA motif within the TMD of EBOV-GP has been associated with GP-mediate cellular detachment [416], raising the question whether this motif could also contribute to EBOV-GP-mediated tetherin antagonism.

My results revealed that the GXXXA motif is not required for EBOV-GP expression, particle incorporation and viral entry, although it should be stated that mutation of the motif reduced entry by about $50 \%$ in Vero E6 and EpoNi/22.1 cells. Moreover, mutation of this motif did not affect the ability of EBOV-GP to promote release of HIV- and EBOV-based viral particles from tetherin-negative cells [373,413,415,434]. In contrast, mutation of this motif abrogated tetherin counteraction by EBOV-GP in the context of spherical HIV Gag- [373,413] and filamentous EBOV VP40-based particles [103,104].

Hacke and colleagues reported that the GXXXA motif and membrane cholesterol are required for GP-mediated cellular detachment. Based on these findings they suggested that the GXXXA motif drives GP-GP homotypic interactions within lipid rafts 
and that this is required for cellular detachment [416]. In contrast to what Hacke and colleagues postulated, a previous study suggested that GP is not located in lipid rafts [435]. Moreover, it was suggested that GP does not alter tetherin localization in lipid rafts [435] and that targeting SGP, which does not counteract tetherin, to lipid rafts is not sufficient for it to mediate tetherin antagonism [414]. My results show that cellular detachment but not tetherin antagonism by a LXXXL mutant of GP (i.e. GXXXA motif mutated) can be rescued by addition of exogenous cholesterol, arguing against an important role of cholesterol in tetherin antagonism. It has been suggested that the interaction between the TMD of single-pass membrane proteins depends on GXXXG or (small amino acid)XXX(small amino acid) motifs, and the presence of these motifs could be used, in some cases, as indicator of TMD oligomerization [436]. This raises the possibility that the GXXXA motif might contribute to formation and/or stability of EBOV-GP trimers, which in turn might be required for tetherin antagonism. However, I obtained no evidence for an impact of the GXXXA motif on trimer stability. As an alternative explanation for the role of the GXXXA motif in tetherin counteraction one could speculate that this domain is required for GP interactions with a cellular factor required for tetherin antagonism and this possibility remains to be investigated.

Two studies demonstrated that EBOV replicates efficiently in tetherin-positive cells $[365,373]$. However, it was not elucidated whether this was due to GP-mediated tetherin antagonism. A replication-competent VSV-EBOV-GP chimera is an EVD vaccine candidate $[42,43]$ and offers the possibility to study whether GP counteracts tetherin in infected cells without having to recur to BSL-4 experiments $[437,438]$. I could show that both, GP wt and the LXXXL mutant, were expressed at similar levels upon infection and were both efficiently incorporated into viral particles. Moreover, mutation of the GXXXA motif had little effect on VSV-EBOV-GP replication in tetherin-negative cells, and the small effect observed might be due to slightly reduced viral entry, as discussed above. Finally, replication of VSV was strongly inhibited by tetherin, in keeping with the finding that this virus does not encode for a tetherin antagonist [439]. Collectively, these results suggest that GP-mediated tetherin counteraction can occur in the context of infected cells, at least when surrogate systems are used. Therefore, mutation of the GXXXA motif in the context of authentic EBOV might allow to determine whether tetherin antagonism occurs in infected cells and contributes to viral spread. 


\section{Outlook}

During my doctoral thesis I was able to determine that proteolytic priming of EBOV-GP by CatB and CatL is independent of viral particle shape and cell line. A remarkable finding was the identification of Calu- 3 cells as one of the few cell lines that are largely resistant to filovirus GP-driven entry and I could provide evidence that entry is restricted at the stages of attachment and GP priming. Future studies need to determine whether Calu-3 cells are also resistant to entry of authentic EBOV and if entry is enhanced in Calu-3 cells expressing CatL or DC-SIGN. Furthermore, it needs to be elucidated whether co-expression of both proteins further augments entry efficiency. Finally, it will be interesting to determine if usage of certain attachment factors like DC-SIGN might impact CatB/CatL dependence of viral entry potentially by introducing viral particles into different uptake pathways.

My work on the antiviral host cell factor tetherin revealed that a mutation within the TMD domain of EBOV-GP abrogates tetherin antagonism while largely maintaining efficient host cell entry. This finding provided the first evidence that EBOV-GP can antagonize tetherin in the context of an infectious surrogate system. Further studies are required to identify fully selective mutations that have no effect on entry but completely abrogate tetherin antagonism. Moreover, it remains to be determined whether mutating the GXXXA motif in the context of infectious EBOV inhibits tetherin antagonism and whether this inhibition affects viral spread. Finally, it is essential determine how GP counteracts tetherin and how the GXXXA motif contributes to this process. 


\section{References}

1. WHO (World Health Organization) Ebola Virus Disease Fact Sheet Available online: https://www.who.int/news-room/fact-sheets/detail/ebola-virus-disease (accessed on Feb 12, 2018).

2. Ploquin, A.; Zhou, Y.; Sullivan, N.J. Ebola Immunity: Gaining a Winning Position in Lightning Chess. J. Immunol. 2018, 201, 833-842.

3. Leroy, E.M.; Kumulungui, B.; Pourrut, X.; Rouquet, P.; Hassanin, A.; Yaba, P.; Délicat, A.; Paweska, J.T.; Gonzalez, J.-P.; Swanepoel, R. Fruit bats as reservoirs of Ebola virus. Nature 2005, 438, 575-6.

4. $\quad$ Biek, R.; Walsh, P.D.; Leroy, E.M.; Real, L.A. Recent common ancestry of Ebola Zaire virus found in a bat reservoir. PLoS Pathog. 2006, 2, e90.

5. Negredo, A.; Palacios, G.; Vázquez-Morón, S.; González, F.; Dopazo, H.; Molero, F.; Juste, J.; Quetglas, J.; Savji, N.; de la Cruz Martínez, M.; et al. Discovery of an ebolavirus-like filovirus in europe. PLOS Pathog. 2011, 7, e1002304.

6. Goldstein, T.; Anthony, S.J.; Gbakima, A.; Bird, B.H.; Bangura, J.; TremeauBravard, A.; Belaganahalli, M.N.; Wells, H.L.; Dhanota, J.K.; Liang, E.; et al. The discovery of Bombali virus adds further support for bats as hosts of ebolaviruses. Nat. Microbiol. 2018, 3, 1084-1089.

7. Pourrut, X.; Souris, M.; Towner, J.S.; Rollin, P.E.; Nichol, S.T.; Gonzalez, J.-P.; Leroy, E. Large serological survey showing cocirculation of Ebola and Marburg viruses in Gabonese bat populations, and a high seroprevalence of both viruses in Rousettus aegyptiacus. BMC Infect. Dis. 2009, 9, 159.

8. Kuzmin, I. V.; Niezgoda, M.; Franka, R.; Agwanda, B.; Markotter, W.; Breiman, R.F.; Shieh, W.J.; Zaki, S.R.; Rupprecht, C.E. Marburg virus in fruit bat, Kenya. Emerg. Infect. Dis. 2010, 16, 352-4.

9. Leendertz, S.A.J.; Gogarten, J.F.; Düx, A.; Calvignac-Spencer, S.; Leendertz, F.H. Assessing the evidence supporting fruit bats as the primary reservoirs for ebola viruses. Ecohealth 2016, 13, 18-25.

10. Leendertz, S.A.J. Testing new hypotheses regarding ebolavirus reservoirs. Viruses 2016, 8, 30.

11. Chippaux, J.-P. Outbreaks of Ebola virus disease in Africa: the beginnings of a tragic saga. J. Venom. Anim. Toxins Incl. Trop. Dis. 2014, 20, 44.

12. Emanuel, J.; Marzi, A.; Feldmann, H. Filoviruses: Ecology, Molecular Biology, and Evolution. Adv. Virus Res. 2018, 100, 189-221.

13. Mate, S.E.; Kugelman, J.R.; Nyenswah, T.G.; Ladner, J.T.; Wiley, M.R.; CordierLassalle, T.; Christie, A.; Schroth, G.P.; Gross, S.M.; Davies-Wayne, G.J.; et al. Molecular Evidence of Sexual Transmission of Ebola Virus. N. Engl. J. Med. 2015, 373, 2448-54.

14. Christie, A.; Davies-Wayne, G.J.; Cordier-Lassalle, T.; Cordier-Lasalle, T.; Blackley, D.J.; Laney, A.S.; Williams, D.E.; Shinde, S.A.; Badio, M.; Lo, T.; et al. Possible sexual transmission of Ebola virus - Liberia, 2015. MMWR. Morb. Mortal. Wkly. Rep. 2015, 64, 479-81.

15. Diallo, B.; Sissoko, D.; Loman, N.J.; Bah, H.A.; Bah, H.; Worrell, M.C.; Conde, L.S.; Sacko, R.; Mesfin, S.; Loua, A.; et al. Resurgence of Ebola Virus Disease in Guinea Linked to a Survivor With Virus Persistence in Seminal Fluid for More Than 500 Days. Clin. Infect. Dis. 2016, 63, 1353-1356.

16. Fischer, W.A.; Brown, J.; Wohl, D.A.; Loftis, A.J.; Tozay, S.; Reeves, E.; Pewu, 
K.; Gorvego, G.; Quellie, S.; Cunningham, C.K.; et al. Ebola Virus Ribonucleic Acid Detection in Semen More Than Two Years After Resolution of Acute Ebola Virus Infection. Open forum Infect. Dis. 2017, 4, ofx155.

17. Subissi, L.; Keita, M.; Mesfin, S.; Rezza, G.; Diallo, B.; Van Gucht, S.; Musa, E.O.; Yoti, Z.; Keita, S.; Djingarey, M.H.; et al. Ebola Virus Transmission Caused by Persistently Infected Survivors of the 2014-2016 Outbreak in West Africa. J. Infect. Dis. 2018, 218, S287-S291.

18. CDC (Centers for Disease Control and Prevention) Ebola (Ebola Virus Disease) Available online: https://www.cdc.gov/vhf/ebola/index.html.

19. Kortepeter, M.G.; Bausch, D.G.; Bray, M. Basic clinical and laboratory features of filoviral hemorrhagic fever. J. Infect. Dis. 2011, 204 Suppl, S810-6.

20. Chertow, D.S.; Kleine, C.; Edwards, J.K.; Scaini, R.; Giuliani, R.; Sprecher, A. Ebola virus disease in West Africa--clinical manifestations and management. $N$. Engl. J. Med. 2014, 371, 2054-7.

21. Schieffelin, J.S.; Shaffer, J.G.; Goba, A.; Gbakie, M.; Gire, S.K.; Colubri, A.; Sealfon, R.S.G.; Kanneh, L.; Moigboi, A.; Momoh, M.; et al. Clinical illness and outcomes in patients with Ebola in Sierra Leone. N. Engl. J. Med. 2014, 371, 2092-100.

22. Uyeki, T.M.; Mehta, A.K.; Davey, R.T.; Liddell, A.M.; Wolf, T.; Vetter, P.; Schmiedel, S.; Grünewald, T.; Jacobs, M.; Arribas, J.R.; et al. Clinical Management of Ebola Virus Disease in the United States and Europe. N. Engl. J. Med. 2016.

23. McElroy, A.K.; Erickson, B.R.; Flietstra, T.D.; Rollin, P.E.; Nichol, S.T.; Towner, J.S.; Spiropoulou, C.F. Ebola hemorrhagic Fever: novel biomarker correlates of clinical outcome. J. Infect. Dis. 2014, 210, 558-66.

24. Bwaka, M.A.; Bonnet, M.J.; Calain, P.; Colebunders, R.; De Roo, A.; Guimard, Y.; Katwiki, K.R.; Kibadi, K.; Kipasa, M.A.; Kuvula, K.J.; et al. Ebola hemorrhagic fever in Kikwit, Democratic Republic of the Congo: clinical observations in 103 patients. J. Infect. Dis. 1999, 179 Suppl, S1-7.

25. Okware, S.I.; Omaswa, F.G.; Zaramba, S.; Opio, A.; Lutwama, J.J.; Kamugisha, J.; Rwaguma, E.B.; Kagwa, P.; Lamunu, M. An outbreak of Ebola in Uganda. Trop. Med. Int. Health 2002, 7, 1068-75.

26. Hunt, L.; Gupta-Wright, A.; Simms, V.; Tamba, F.; Knott, V.; Tamba, K.; Heisenberg-Mansaray, S.; Tamba, E.; Sheriff, A.; Conteh, S.; et al. Clinical presentation, biochemical, and haematological parameters and their association with outcome in patients with Ebola virus disease: an observational cohort study. Lancet. Infect. Dis. 2015, 15, 1292-9.

27. Deen, G.F.; Broutet, N.; Xu, W.; Knust, B.; Sesay, F.R.; McDonald, S.L.R.; Ervin, E.; Marrinan, J.E.; Gaillard, P.; Habib, N.; et al. Ebola RNA Persistence in Semen of Ebola Virus Disease Survivors - Final Report. N. Engl. J. Med. 2017, 377, 1428-1437.

28. Varkey, J.B.; Shantha, J.G.; Crozier, I.; Kraft, C.S.; Lyon, G.M.; Mehta, A.K.; Kumar, G.; Smith, J.R.; Kainulainen, M.H.; Whitmer, S.; et al. Persistence of Ebola Virus in Ocular Fluid during Convalescence. N. Engl. J. Med. 2015, 372, 2423-7.

29. Jacobs, M.; Rodger, A.; Bell, D.J.; Bhagani, S.; Cropley, I.; Filipe, A.; Gifford, R.J.; Hopkins, S.; Hughes, J.; Jabeen, F.; et al. Late Ebola virus relapse causing meningoencephalitis: a case report. Lancet (London, England) 2016, 388, 498503.

30. Sissoko, D.; Keïta, M.; Diallo, B.; Aliabadi, N.; Fitter, D.L.; Dahl, B.A.; Akoi Bore, 
J.; Raymond Koundouno, F.; Singethan, K.; Meisel, S.; et al. Ebola Virus Persistence in Breast Milk After No Reported Illness: A Likely Source of Virus Transmission From Mother to Child. Clin. Infect. Dis. 2017, 64, 513-516.

31. Wong, G.; Gao, G.F.; Qiu, X. Can Ebola virus become endemic in the human population? Protein Cell 2016, 7, 4-6.

32. Lupton, H.W.; Lambert, R.D.; Bumgardner, D.L.; Moe, J.B.; Eddy, G.A. Inactivated vaccine for Ebola virus efficacious in guineapig model. Lancet (London, England) 1980, 2, 1294-5.

33. Blaney, J.E.; Wirblich, C.; Papaneri, A.B.; Johnson, R.F.; Myers, C.J.; Juelich, T.L.; Holbrook, M.R.; Freiberg, A.N.; Bernbaum, J.G.; Jahrling, P.B.; et al. Inactivated or live-attenuated bivalent vaccines that confer protection against rabies and Ebola viruses. J. Virol. 2011, 85, 10605-16.

34. Warfield, K.L.; Posten, N.A.; Swenson, D.L.; Olinger, G.G.; Esposito, D.; Gillette, W.K.; Hopkins, R.F.; Costantino, J.; Panchal, R.G.; Hartley, J.L.; et al. Filoviruslike particles produced in insect cells: immunogenicity and protection in rodents. J. Infect. Dis. 2007, 196 Suppl, S421-9.

35. Halperin, S.A.; Arribas, J.R.; Rupp, R.; Andrews, C.P.; Chu, L.; Das, R.; Simon, J.K.; Onorato, M.T.; Liu, K.; Martin, J.; et al. Six-Month Safety Data of Recombinant Vesicular Stomatitis Virus-Zaire Ebola Virus Envelope Glycoprotein Vaccine in a Phase 3 Double-Blind, Placebo-Controlled Randomized Study in Healthy Adults. J. Infect. Dis. 2017, 215, 1789-1798.

36. Huttner, A.; Dayer, J.-A.; Yerly, S.; Combescure, C.; Auderset, F.; Desmeules, J.; Eickmann, M.; Finckh, A.; Goncalves, A.R.; Hooper, J.W.; et al. The effect of dose on the safety and immunogenicity of the VSV Ebola candidate vaccine: a randomised double-blind, placebo-controlled phase 1/2 trial. Lancet. Infect. Dis. 2015, 15, 1156-1166.

37. Agnandji, S.T.; Huttner, A.; Zinser, M.E.; Njuguna, P.; Dahlke, C.; Fernandes, J.F.; Yerly, S.; Dayer, J.-A.; Kraehling, V.; Kasonta, R.; et al. Phase 1 Trials of rVSV Ebola Vaccine in Africa and Europe. N. Engl. J. Med. 2016, 374, 1647-60.

38. Henao-Restrepo, A.M.; Camacho, A.; Longini, I.M.; Watson, C.H.; Edmunds, W.J.; Egger, M.; Carroll, M.W.; Dean, N.E.; Diatta, I.; Doumbia, M.; et al. Efficacy and effectiveness of an rVSV-vectored vaccine in preventing Ebola virus disease: final results from the Guinea ring vaccination, open-label, clusterrandomised trial (Ebola Ça Suffit!). Lancet (London, England) 2017, 389, 505518.

39. Marzi, A.; Robertson, S.J.; Haddock, E.; Feldmann, F.; Hanley, P.W.; Scott, D.P.; Strong, J.E.; Kobinger, G.; Best, S.M.; Feldmann, H. EBOLA VACCINE. VSVEBOV rapidly protects macaques against infection with the 2014/15 Ebola virus outbreak strain. Science 2015, 349, 739-42.

40. Venkatraman, N.; Silman, D.; Folegatti, P.M.; Hill, A.V.S. Vaccines against Ebola virus. Vaccine 2018, 36, 5454-5459.

41. Dhama, K.; Karthik, K.; Khandia, R.; Chakraborty, S.; Munjal, A.; Latheef, S.K.; Kumar, D.; Ramakrishnan, M.A.; Malik, Y.S.; Singh, R.; et al. Advances in Designing and Developing Vaccines, Drugs, and Therapies to Counter Ebola Virus. Front. Immunol. 2018, 9, 1803.

42. Geisbert, T.W.; Feldmann, H. Recombinant vesicular stomatitis virus-based vaccines against Ebola and Marburg virus infections. J. Infect. Dis. 2011, 204 Suppl, S1075-81.

43. Wong, G.; Qiu, X. Designing Efficacious Vesicular Stomatitis Virus-Vectored Vaccines Against Ebola Virus. Methods Mol. Biol. 2016, 1403, 245-57. 
44. Dolzhikova, I. V.; Zubkova, O. V.; Tukhvatulin, A.I.; Dzharullaeva, A.S.; Tukhvatulina, N.M.; Shcheblyakov, D. V.; Shmarov, M.M.; Tokarskaya, E.A.; Simakova, Y. V.; Egorova, D.A.; et al. Safety and immunogenicity of GamEvacCombi, a heterologous VSV- and Ad5-vectored Ebola vaccine: An open phase I/II trial in healthy adults in Russia. Hum. Vaccin. Immunother. 2017, 13, 613620.

45. Zhu, F.-C.; Hou, L.-H.; Li, J.-X.; Wu, S.-P.; Liu, P.; Zhang, G.-R.; Hu, Y.-M.; Meng, F.-Y.; Xu, J.-J.; Tang, R.; et al. Safety and immunogenicity of a novel recombinant adenovirus type-5 vector-based Ebola vaccine in healthy adults in China: preliminary report of a randomised, double-blind, placebo-controlled, phase 1 trial. Lancet (London, England) 2015, 385, 2272-9.

46. Wong, G.; Mendoza, E.J.; Plummer, F.A.; Gao, G.F.; Kobinger, G.P.; Qiu, X. From bench to almost bedside: the long road to a licensed Ebola virus vaccine. Expert Opin. Biol. Ther. 2018, 18, 159-173.

47. Mupapa, K.; Massamba, M.; Kibadi, K.; Kuvula, K.; Bwaka, A.; Kipasa, M.; Colebunders, R.; Muyembe-Tamfum, J.J. Treatment of Ebola hemorrhagic fever with blood transfusions from convalescent patients. International Scientific and Technical Committee. J. Infect. Dis. 1999, 179 Suppl, S18-23.

48. Gulland, A. First Ebola treatment is approved by WHO. BMJ 2014, 349, g5539.

49. Sahr, F.; Ansumana, R.; Massaquoi, T.A.; Idriss, B.R.; Sesay, F.R.; Lamin, J.M.; Baker, S.; Nicol, S.; Conton, B.; Johnson, W.; et al. Evaluation of convalescent whole blood for treating Ebola Virus Disease in Freetown, Sierra Leone. J. Infect. 2017, 74, 302-309.

50. van Griensven, J.; De Weiggheleire, A.; Delamou, A.; Smith, P.G.; Edwards, T.; Vandekerckhove, P.; Bah, E.I.; Colebunders, R.; Herve, I.; Lazaygues, C.; et al. The Use of Ebola Convalescent Plasma to Treat Ebola Virus Disease in Resource-Constrained Settings: A Perspective From the Field. Clin. Infect. Dis. 2016, 62, 69-74.

51. van Griensven, J.; Edwards, T.; Baize, S.; Ebola-Tx Consortium Efficacy of Convalescent Plasma in Relation to Dose of Ebola Virus Antibodies. N. Engl. J. Med. 2016, 375, 2307-2309.

52. Garraud, O. Use of convalescent plasma in Ebola virus infection. Transfus. Apher. Sci. 2017, 56, 31-34.

53. Geisen, C.; Kann, G.; Strecker, T.; Wolf, T.; Schüttfort, G.; van Kraaij, M.; MacLennan, S.; Rummler, S.; Weinigel, C.; Eickmann, M.; et al. Pathogenreduced Ebola virus convalescent plasma: first steps towards standardization of manufacturing and quality control including assessment of Ebola-specific neutralizing antibodies. Vox Sang. 2016, 110, 329-35.

54. Qiu, X.; Wong, G.; Audet, J.; Bello, A.; Fernando, L.; Alimonti, J.B.; FaustherBovendo, H.; Wei, H.; Aviles, J.; Hiatt, E.; et al. Reversion of advanced Ebola virus disease in nonhuman primates with ZMapp. Nature 2014, 514, 47-53.

55. Zhang, Y.; Li, D.; Jin, X.; Huang, Z. Fighting Ebola with ZMapp: spotlight on plantmade antibody. Sci. China. Life Sci. 2014, 57, 987-8.

56. Trad, M.A.; Naughton, W.; Yeung, A.; Mazlin, L.; O'sullivan, M.; Gilroy, N.; Fisher, D.A.; Stuart, R.L. Ebola virus disease: An update on current prevention and management strategies. J. Clin. Virol. 2017, 86, 5-13.

57. Maruyama, T.; Rodriguez, L.L.; Jahrling, P.B.; Sanchez, A.; Khan, A.S.; Nichol, S.T.; Peters, C.J.; Parren, P.W.; Burton, D.R. Ebola virus can be effectively neutralized by antibody produced in natural human infection. J. Virol. 1999, 73, 6024-30. 
58. Parren, P.W.H.I.; Geisbert, T.W.; Maruyama, T.; Jahrling, P.B.; Burton, D.R. Preand postexposure prophylaxis of Ebola virus infection in an animal model by passive transfer of a neutralizing human antibody. J. Virol. 2002, 76, 6408-12.

59. Hiatt, A.; Pauly, M.; Whaley, K.; Qiu, X.; Kobinger, G.; Zeitlin, L. The emergence of antibody therapies for Ebola. Hum. Antibodies 2015, 23, 49-56.

60. Fischer, W.A.; Vetter, P.; Bausch, D.G.; Burgess, T.; Davey, R.T.; Fowler, R.; Hayden, F.G.; Jahrling, P.B.; Kalil, A.C.; Mayers, D.L.; et al. Ebola virus disease: an update on post-exposure prophylaxis. Lancet Infect. Dis. 2018, 18, e183e192.

61. Razaghi, A.; Owens, L.; Heimann, K. Review of the recombinant human interferon gamma as an immunotherapeutic: Impacts of production platforms and glycosylation. J. Biotechnol. 2016.

62. Jerebtsova, M.; Nekhai, S. Therapeutics for postexposure treatment of Ebola virus infection. Future Virol. 2015, 10, 221-232.

63. Zhao, Y.; Ren, J.; Harlos, K.; Jones, D.M.; Zeltina, A.; Bowden, T.A.; PadillaParra, S.; Fry, E.E.; Stuart, D.I. Toremifene interacts with and destabilizes the Ebola virus glycoprotein. Nature 2016, 535, 169-172.

64. Nelson, E.A.; Barnes, A.B.; Wiehle, R.D.; Fontenot, G.K.; Hoenen, T.; White, J.M. Clomiphene and Its Isomers Block Ebola Virus Particle Entry and Infection with Similar Potency: Potential Therapeutic Implications. Viruses 2016, 8.

65. Manabe, T.; Hirano, T.; Imanishi, K.; Ando, K.; Yotsumoto, F.; Tobe, T. Protective effect of nafamostat mesilate on cellular and lysosomal fragility of acinar cells in rat cerulein pancreatitis. Int. J. Pancreatol. 1992, 12, 167-72.

66. Nishimura, H.; Yamaya, M. A Synthetic Serine Protease Inhibitor, Nafamostat Mesilate, Is a Drug Potentially Applicable to the Treatment of Ebola Virus Disease. Tohoku J. Exp. Med. 2015, 237, 45-50.

67. WHO (World Health Organization) Ebola Virus Disease 2014-2016 Outbreak Available online: https://www.who.int/csr/disease/ebola/en/.

68. WHO (World Health Organization) Ebola Virus Disease: situation reports 20172018 Available online: http://www.afro.who.int/health-topics/ebola-virusdisease.

69. WHO (World Health Organization) Eboba Virus Disease: Current Reports Available online: https://www.who.int/ebola/situation-reports/drc-2018/en/.

70. ICTV (International Committee on Taxonomy of Viruses) Virus Taxonomy Available online: https://talk.ictvonline.org/taxonomy/.

71. Cross, R.W.; Mire, C.E.; Feldmann, H.; Geisbert, T.W. Post-exposure treatments for Ebola and Marburg virus infections. Nat. Rev. Drug Discov. 2018, 17, 413434.

72. CDC (Centers for Disease Control and Prevention) Marburg hemorrhagic fever (Marburg HF)-Outbreaks Available online: https://www.cdc.gov/vhf/marburg/outbreaks/index.html.

73. Feldmann, H.; Geisbert, T.W. Filoviridae: Marburg and Ebola Viruses. In Field's Virology; Knipe, D.., Howley, P.M., Eds.; Philadelphia: Wolters Kluwer Health/Lippincott Williams \& Wilkins, 2013; pp. 923-56 ISBN 9781469830667.

74. Hoenen, T.; Feldmann, H. Reverse genetics systems as tools for the development of novel therapies against filoviruses. Expert Rev. Anti. Infect. Ther. 2014, 12, 1253-63.

75. Banadyga, L.; Dolan, M.A.; Ebihara, H. Rodent-Adapted Filoviruses and the Molecular Basis of Pathogenesis. J. Mol. Biol. 2016, 428, 3449-66.

76. Volchkov, V.E.; Becker, S.; Volchkova, V.A.; Ternovoj, V.A.; Kotov, A.N.; 
Netesov, S. V; Klenk, H.D. GP mRNA of Ebola virus is edited by the Ebola virus polymerase and by T7 and vaccinia virus polymerases. Virology 1995, 214, 42130.

77. Baseler, L.; Chertow, D.S.; Johnson, K.M.; Feldmann, H.; Morens, D.M. The Pathogenesis of Ebola Virus Disease. Annu. Rev. Pathol. Mech. Dis. 2017, 12, 387-418.

78. Huang, Y.; Xu, L.; Sun, Y.; Nabel, G.J. The assembly of Ebola virus nucleocapsid requires virion-associated proteins 35 and 24 and posttranslational modification of nucleoprotein. Mol. Cell 2002, 10, 307-16.

79. Han, Z.; Boshra, H.; Sunyer, J.O.; Zwiers, S.H.; Paragas, J.; Harty, R.N. Biochemical and functional characterization of the Ebola virus VP24 protein: implications for a role in virus assembly and budding. J. Virol. 2003, 77, 1793800.

80. Ruigrok, R.W.; Schoehn, G.; Dessen, A.; Forest, E.; Volchkov, V.; Dolnik, O.; Klenk, H.D.; Weissenhorn, W. Structural characterization and membrane binding properties of the matrix protein VP40 of Ebola virus. J. Mol. Biol. 2000, 300, 10312.

81. Pleet, M.L.; DeMarino, C.; Lepene, B.; Aman, M.J.; Kashanchi, F. The Role of Exosomal VP40 in Ebola Virus Disease. DNA Cell Biol. 2017, 36, 243-248.

82. Lee, J.E.; Saphire, E.O. Ebolavirus glycoprotein structure and mechanism of entry. Future Virol. 2009, 4, 621-635.

83. SIB (Swiss Institute of Bioinformatics) ViralZone Available online: www.expasy.org/viralzone (accessed on Nov 30, 2018).

84. Aleksandrowicz, P.; Marzi, A.; Biedenkopf, N.; Beimforde, N.; Becker, S.; Hoenen, T.; Feldmann, H.; Schnittler, H.J. Ebola virus enters host cells by macropinocytosis and clathrin-mediated endocytosis. J. Infect. Dis. 2011, 204, S957-S967.

85. Côté, M.; Misasi, J.; Ren, T.; Bruchez, A.; Lee, K.; Filone, C.M.; Hensley, L.; Li, Q.; Ory, D.; Chandran, K.; et al. Small molecule inhibitors reveal Niemann-Pick $\mathrm{C} 1$ is essential for Ebola virus infection. Nature 2011, 477, 344.

86. Carette, J.E.; Raaben, M.; Wong, A.C.; Herbert, A.S.; Obernosterer, G.; Mulherkar, N.; Kuehne, A.I.; Kranzusch, P.J.; Griffin, A.M.; Ruthel, G.; et al. Ebola virus entry requires the cholesterol transporter Niemann-Pick C1. Nature 2011, 477, 340-3.

87. White, J.M.; Schornberg, K.L. A new player in the puzzle of filovirus entry. Nat. Rev. Microbiol. 2012, 10, 317-322.

88. Markosyan, R.M.; Miao, C.; Zheng, Y.M.; Melikyan, G.B.; Liu, S.L.; Cohen, F.S. Induction of Cell-Cell Fusion by Ebola Virus Glycoprotein: Low pH Is Not a Trigger. PLoS Pathog. 2016, 12, 1-31.

89. Lee, J.; Gregory, S.M.; Nelson, E.A.; White, J.M.; Tamm, L.K. The roles of histidines and charged residues as potential triggers of a conformational change in the fusion loop of ebola virus glycoprotein. PLoS One 2016.

90. White, J.M.; Delos, S.E.; Brecher, M.; Schornberg, K. Structures and mechanisms of viral membrane fusion proteins: multiple variations on a common theme. Crit. Rev. Biochem. Mol. Biol. 2008, 43, 189-219.

91. Weissenhorn, W.; Carfí, A.; Lee, K.H.; Skehel, J.J.; Wiley, D.C. Crystal structure of the Ebola virus membrane fusion subunit, GP2, from the envelope glycoprotein ectodomain. Mol. Cell 1998, 2, 605-16.

92. Malashkevich, V.N.; Schneider, B.J.; McNally, M.L.; Milhollen, M.A.; Pang, J.X.; Kim, P.S. Core structure of the envelope glycoprotein GP2 from Ebola virus at 
1.9-A resolution. Proc. Natl. Acad. Sci. U. S. A. 1999, 96, 2662-7.

93. Lee, J.E.; Fusco, M.L.; Hessell, A.J.; Oswald, W.B.; Burton, D.R.; Saphire, E.O. Structure of the Ebola virus glycoprotein bound to an antibody from a human survivor. Nature 2008, 454, 177-82.

94. Gregory, S.M.; Harada, E.; Liang, B.; Delos, S.E.; White, J.M.; Tamm, L.K. Structure and function of the complete internal fusion loop from Ebolavirus glycoprotein 2. Proc. Natl. Acad. Sci. U. S. A. 2011, 108, 11211-6.

95. Gregory, S.M.; Larsson, P.; Nelson, E.A.; Kasson, P.M.; White, J.M.; Tamm, L.K. Ebolavirus entry requires a compact hydrophobic fist at the tip of the fusion loop. J. Virol. 2014, 88, 6636-49.

96. Martin, B.; Hoenen, T.; Canard, B.; Decroly, E. Filovirus proteins for antiviral drug discovery: A structure/function analysis of surface glycoproteins and virus entry. Antiviral Res. 2016, 135, 1-14.

97. Yu, D.-S.; Weng, T.-H.; Wu, X.-X.; Wang, F.X.C.; Lu, X.-Y.; Wu, H.-B.; Wu, N.P.; Li, L.-J.; Yao, H.-P. The lifecycle of the Ebola virus in host cells. Oncotarget 2017, 8, 55750-55759.

98. Ruigrok, R.W.H.; Crépin, T.; Kolakofsky, D. Nucleoproteins and nucleocapsids of negative-strand RNA viruses. Curr. Opin. Microbiol. 2011, 14, 504-10.

99. Noda, T.; Ebihara, H.; Muramoto, Y.; Fujii, K.; Takada, A.; Sagara, H.; Kim, J.H.; Kida, H.; Feldmann, H.; Kawaoka, Y. Assembly and budding of Ebolavirus. PLoS Pathog. 2006, 2, e99.

100. Schudt, G.; Dolnik, O.; Kolesnikova, L.; Biedenkopf, N.; Herwig, A.; Becker, S. Transport of Ebolavirus Nucleocapsids Is Dependent on Actin Polymerization: Live-Cell Imaging Analysis of Ebolavirus-Infected Cells. J. Infect. Dis. 2015, 212 Suppl, S160-6.

101. Ito, H.; Watanabe, S.; Takada, A.; Kawaoka, Y. Ebola Virus Glycoprotein: Proteolytic Processing, Acylation, Cell Tropism, and Detection of Neutralizing Antibodies. J. Virol. 2001, 75, 1576-1580.

102. Jeffers, S.A.; Sanders, D.A.; Sanchez, A. Covalent modifications of the ebola virus glycoprotein. J. Virol. 2002, 76, 12463-72.

103. Bavari, S.; Bosio, C.; Wiegand, E.; Ruthel, G.; Will, A.; Geisbert, T.; Hevey, M.; Schmaljohn, C.; Schmaljohn, A.; Aman, M. Lipid raft microdomains: a gateway for compartmentalized trafficking of Ebola and Marburg viruses. J. Exp. Med. 2002, 195, 593-602.

104. Noda, T.; Sagara, H.; Suzuki, E.; Takada, A.; Kida, H.; Kawaoka, Y. Ebola virus VP40 drives the formation of virus-like filamentous particles along with GP. J. Virol. 2002, 76, 4855-65.

105. Timmins, J.; Schoehn, G.; Kohlhaas, C.; Klenk, H.-D.; Ruigrok, R.W.H.; Weissenhorn, W. Oligomerization and polymerization of the filovirus matrix protein VP40. Virology 2003, 312, 359-68.

106. Bornholdt, Z.A.; Noda, T.; Abelson, D.M.; Halfmann, P.; Wood, M.R.; Kawaoka, Y.; Saphire, E.O. Structural rearrangement of ebola virus VP40 begets multiple functions in the virus life cycle. Cell 2013, 154, 763-74.

107. Soni, S.P.; Stahelin, R. V. The ebola virus matrix protein VP40 selectively induces vesiculation from phosphatidylserine-enriched membranes. J. Biol. Chem. 2014.

108. Mehedi, M.; Falzarano, D.; Seebach, J.; Hu, X.; Carpenter, M.S.; Schnittler, H.J.; Feldmann, H. A New Ebola Virus Nonstructural Glycoprotein Expressed through RNA Editing. J. Virol. 2011, 85, 5406-5414.

109. Cook, J.D.; Lee, J.E. The secret life of viral entry glycoproteins: moonlighting in 
immune evasion. PLoS Pathog. 2013, 9, e1003258.

110. Volchkova, V. a.; Klenk, H.-D.; Volchkov, V.E. Delta-peptide is the carboxyterminal cleavage fragment of the nonstructural small glycoprotein sGP of Ebola virus. Virology 1999, 265, 164-171.

111. Barrientos, L.G.; Martin, A.M.; Rollin, P.E.; Sanchez, A. Disulfide bond assignment of the Ebola virus secreted glycoprotein SGP. Biochem. Biophys. Res. Commun. 2004, 323, 696-702.

112. Falzarano, D.; Krokhin, O.; Wahl-Jensen, V.; Seebach, J.; Wolf, K.; Schnittler, H.J.; Feldmann, H. Structure-function analysis of the soluble glycoprotein, sGP, of ebola virus. ChemBioChem 2006, 7, 1605-1611.

113. Mohan, G.S.; Li, W.; Ye, L.; Compans, R.W.; Yang, C. Antigenic subversion: a novel mechanism of host immune evasion by Ebola virus. PLoS Pathog. 2012, 8, e1003065.

114. Sui, J.; Marasco, W.A. Evidence against Ebola virus sGP binding to human neutrophils by a specific receptor. Virology 2002, 303, 9-14.

115. Wahl-Jensen, V.M.; Afanasieva, T.A.; Seebach, J.; Ströher, U.; Feldmann, H.; Schnittler, H.-J. Effects of Ebola virus glycoproteins on endothelial cell activation and barrier function. J. Virol. 2005, 79, 10442-50.

116. Iwasa, A.; Shimojima, M.; Kawaoka, Y. sGP serves as a structural protein in Ebola virus infection. J. Infect. Dis. 2011, 204 Suppl, S897-903.

117. Mohan, G.S.; Ye, L.; Li, W.; Monteiro, A.; Lin, X.; Sapkota, B.; Pollack, B.P.; Compans, R.W.; Yang, C. Less Is More: Ebola Virus Surface Glycoprotein Expression Levels Regulate Virus Production and Infectivity. J. Virol. 2015, 89, 1205-1217.

118. Radoshitzky, S.R.; Warfield, K.L.; Chi, X.; Dong, L.; Kota, K.; Bradfute, S.B.; Gearhart, J.D.; Retterer, C.; Kranzusch, P.J.; Misasi, J.N.; et al. Ebolavirus deltapeptide immunoadhesins inhibit marburgvirus and ebolavirus cell entry. J. Virol. 2011, 85, 8502-13.

119. Gallaher, W.R.; Garry, R.F. Modeling of the Ebola virus delta peptide reveals a potential lytic sequence motif. Viruses 2015, 7, 285-305.

120. Sanchez, A.; Trappier, S.G.; Mahy, B.W.; Peters, C.J.; Nichol, S.T. The virion glycoproteins of Ebola viruses are encoded in two reading frames and are expressed through transcriptional editing. Proc. Natl. Acad. Sci. 1996, 93, 36023607.

121. Ning, Y.J.; Deng, F.; Hu, Z.; Wang, H. The roles of ebolavirus glycoproteins in viral pathogenesis. Virol. Sin. 2017, 32, 3-15.

122. Volchkov, V.E.; Feldmann, H.; Volchkova, V.A.; Klenk, H.-D. Processing of the Ebola virus glycoprotein by the proprotein convertase furin. Proc. Natl. Acad. Sci. 1998, 95, 5762-5767.

123. Sanchez, A.; Yang, Z.-Y.; Xu, L.; Nabel, G.J.; Crews, T.; Peters, C.J. Biochemical analysis of the secreted and virion glycoproteins of ebola virus. $J$. Virol. 1998, 72, 6442-6447.

124. Dolnik, O.; Volchkova, V.; Garten, W.; Carbonnelle, C.; Becker, S.; Kahnt, J.; Ströher, U.; Klenk, H.D.; Volchkov, V. Ectodomain shedding of the glycoprotein GP of Ebola virus. EMBO J. 2004, 23, 2175-2184.

125. Escudero-Pérez, B.; Volchkova, V.A.; Dolnik, O.; Lawrence, P.; Volchkov, V.E. Shed GP of Ebola Virus Triggers Immune Activation and Increased Vascular Permeability. PLoS Pathog. 2014, 10, e1004509.

126. Zhao, D.; Han, X.; Zheng, X.; Wang, H.; Yang, Z.; Liu, D.; Han, K.; Liu, J.; Wang, X.; Yang, W.; et al. The Myeloid LSECtin Is a DAP12-Coupled Receptor That Is 
Crucial for Inflammatory Response Induced by Ebola Virus Glycoprotein. PLoS Pathog. 2016, 12, e1005487.

127. Dolnik, O.; Volchkova, V.A.; Escudero-Perez, B.; Lawrence, P.; Klenk, H.D.; Volchkov, V.E. Shedding of Ebola Virus Surface Glycoprotein Is a Mechanism of Self-regulation of Cellular Cytotoxicity and Has a Direct Effect on Virus Infectivity. J. Infect. Dis. 2015, 212, S322-S328.

128. Hoffmann, M.; Crone, L.; Dietzel, E.; Paijo, J.; González-Hernández, M.; Nehlmeier, I.; Kalinke, U.; Becker, S.; Pöhlmann, S. A Polymorphism within the Internal Fusion Loop of the Ebola Virus Glycoprotein Modulates Host Cell Entry. J. Virol. 2017, 91, e00177-17.

129. Bornholdt, Z.A.; Turner, H.L.; Murin, C.D.; Li, W.; Sok, D.; Souders, C.A.; Piper, A.E.; Goff, A.; Shamblin, J.D.; Wollen, S.E.; et al. Isolation of potent neutralizing antibodies from a survivor of the 2014 Ebola virus outbreak. Science 2016, 351, 1078-83.

130. Olejnik, J.; Ryabchikova, E.; Corley, R.B.; Mühlberger, E. Intracellular events and cell fate in filovirus infection. Viruses 2011, 3, 1501-31.

131. Martinez, O.; Valmas, C.; Basler, C.F. Ebola virus-like particle-induced activation of NF-kappaB and Erk signaling in human dendritic cells requires the glycoprotein mucin domain. Virology 2007, 364, 342-54.

132. Okumura, A.; Pitha, P.M.; Yoshimura, A.; Harty, R.N. Interaction between Ebola virus glycoprotein and host toll-like receptor 4 leads to induction of proinflammatory cytokines and SOCS1. J. Virol. 2010, 84, 27-33.

133. Yang, Z.Y.; Duckers, H.J.; Sullivan, N.J.; Sanchez, A.; Nabel, E.G.; Nabel, G.J. Identification of the Ebola virus glycoprotein as the main viral determinant of vascular cell cytotoxicity and injury. Nat. Med. 2000, 6, 886-9.

134. Simmons, G.; Wool-Lewis, R.J.; Baribaud, F.; Netter, R.C.; Bates, P. Ebola Virus Glycoproteins Induce Global Surface Protein Down-Modulation and Loss of Cell Adherence. J. Virol. 2002, 76, 2518-2528.

135. Takada, A.; Watanabe, S.; Ito, H.; Okazaki, K.; Kida, H.; Kawaoka, Y. Downregulation of beta1 integrins by Ebola virus glycoprotein: implication for virus entry. Virology 2000, 278, 20-6.

136. Reynard, O.; Borowiak, M.; Volchkova, V.A.; Delpeut, S.; Mateo, M.; Volchkov, V.E. Ebolavirus glycoprotein GP masks both its own epitopes and the presence of cellular surface proteins. J. Virol. 2009, 83, 9596-601.

137. Francica, J.R.; Varela-Rohena, A.; Medvec, A.; Plesa, G.; Riley, J.L.; Bates, P. Steric shielding of surface epitopes and impaired immune recognition induced by the ebola virus glycoprotein. PLoS Pathog. 2010, 6, e1001098.

138. Harcourt, B.H.; Sanchez, A.; Offermann, M.K. Ebola virus selectively inhibits responses to interferons, but not to interleukin-1beta, in endothelial cells. J. Virol. 1999, 73, 3491-6.

139. Francica, J.R.; Matukonis, M.K.; Bates, P. Requirements for cell rounding and surface protein down-regulation by Ebola virus glycoprotein. Virology 2009, 383, 237-47.

140. Wilson, J.A.; Hevey, M.; Bakken, R.; Guest, S.; Bray, M.; Schmaljohn, A.L.; Hart, M.K. Epitopes involved in antibody-mediated protection from Ebola virus. Science 2000, 287, 1664-6.

141. Martinez, O.; Tantral, L.; Mulherkar, N.; Chandran, K.; Basler, C.F. Impact of Ebola mucin-like domain on antiglycoprotein antibody responses induced by Ebola virus-like particles. J. Infect. Dis. 2011, 204, 825-832.

142. Takada, A. Filovirus tropism: cellular molecules for viral entry. Front. Microbiol. 
2012, 3, 34.

143. Jemielity, S.; Wang, J.J.; Chan, Y.K.; Ahmed, A.A.; Li, W.; Monahan, S.; Bu, X.; Farzan, M.; Freeman, G.J.; Umetsu, D.T.; et al. TIM-family proteins promote infection of multiple enveloped viruses through virion-associated phosphatidylserine. PLoS Pathog. 2013, 9, e1003232.

144. Moller-Tank, S.; Kondratowicz, A.S.; Davey, R.A.; Rennert, P.D.; Maury, W. Role of the phosphatidylserine receptor TIM-1 in enveloped-virus entry. J. Virol. 2013, 87, 8327-41.

145. Vanlandschoot, P.; Leroux-Roels, G. Viral apoptotic mimicry: an immune evasion strategy developed by the hepatitis B virus? Trends Immunol. 2003, 24, 144-7.

146. Mercer, J.; Helenius, A. Vaccinia virus uses macropinocytosis and apoptotic mimicry to enter host cells. Science 2008, 320, 531-5.

147. Moller-Tank, S.; Maury, W. Phosphatidylserine receptors: enhancers of enveloped virus entry and infection. Virology 2014, 468-470, 565-580.

148. Morizono, K.; Xie, Y.; Olafsen, T.; Lee, B.; Dasgupta, A.; Wu, A.M.; Chen, I.S.Y. The soluble serum protein Gas6 bridges virion envelope phosphatidylserine to the TAM receptor tyrosine kinase Axl to mediate viral entry. Cell Host Microbe 2011, 9, 286-98.

149. Meertens, L.; Carnec, X.; Lecoin, M.P.; Ramdasi, R.; Guivel-Benhassine, F.; Lew, E.; Lemke, G.; Schwartz, O.; Amara, A. The TIM and TAM families of phosphatidylserine receptors mediate dengue virus entry. Cell Host Microbe 2012, 12, 544-57.

150. Morizono, K.; Chen, I.S.Y. Role of Phosphatidylserine Receptors in Enveloped Virus Infection. J. Virol. 2014, 88, 4275-4290.

151. Shimojima, M.; Takada, A.; Ebihara, H.; Neumann, G.; Fujioka, K.; Irimura, T.; Jones, S.; Feldmann, H.; Kawaoka, Y. Tyro3 Family-Mediated Cell Entry of Ebola and Marburg Viruses. J. Virol. 2006, 80, 10109-10116.

152. Dahlmann, F.; Biedenkopf, N.; Babler, A.; Jahnen-Dechent, W.; Karsten, C.B.; Gnirß, K.; Schneider, H.; Wrensch, F.; O'Callaghan, C.A.; Bertram, S.; et al. Analysis of Ebola Virus Entry Into Macrophages. J. Infect. Dis. 2015, 212 Suppl, S247-57.

153. Mclntire, J.J.; Umetsu, D.T.; DeKruyff, R.H. TIM-1, a novel allergy and asthma susceptibility gene. Springer Semin. Immunopathol. 2004, 25, 335-48.

154. Kobayashi, N.; Karisola, P.; Peña-Cruz, V.; Dorfman, D.M.; Jinushi, M.; Umetsu, S.E.; Butte, M.J.; Nagumo, H.; Chernova, I.; Zhu, B.; et al. TIM-1 and TIM-4 glycoproteins bind phosphatidylserine and mediate uptake of apoptotic cells. Immunity 2007, 27, 927-40.

155. Miyanishi, M.; Tada, K.; Koike, M.; Uchiyama, Y.; Kitamura, T.; Nagata, S. Identification of Tim4 as a phosphatidylserine receptor. Nature 2007, 450, 4359.

156. DeKruyff, R.H.; Bu, X.; Ballesteros, A.; Santiago, C.; Chim, Y.-L.E.; Lee, H.-H.; Karisola, P.; Pichavant, M.; Kaplan, G.G.; Umetsu, D.T.; et al. T cell/transmembrane, Ig, and mucin-3 allelic variants differentially recognize phosphatidylserine and mediate phagocytosis of apoptotic cells. J. Immunol. 2010, 184, 1918-30.

157. Santiago, C.; Ballesteros, A.; Martínez-Muñoz, L.; Mellado, M.; Kaplan, G.G.; Freeman, G.J.; Casasnovas, J.M. Structures of T cell immunoglobulin mucin protein 4 show a metal-lon-dependent ligand binding site where phosphatidylserine binds. Immunity 2007, 27, 941-51. 
158. Albacker, L.A.; Karisola, P.; Chang, Y.-J.; Umetsu, S.E.; Zhou, M.; Akbari, O.; Kobayashi, N.; Baumgarth, N.; Freeman, G.J.; Umetsu, D.T.; et al. TIM-4, a receptor for phosphatidylserine, controls adaptive immunity by regulating the removal of antigen-specific T cells. J. Immunol. 2010, 185, 6839-49.

159. Lee, H.-H.; Meyer, E.H.; Goya, S.; Pichavant, M.; Kim, H.Y.; Bu, X.; Umetsu, S.E.; Jones, J.C.; Savage, P.B.; Iwakura, Y.; et al. Apoptotic cells activate NKT cells through $\mathrm{T}$ cell Ig-like mucin-like-1 resulting in airway hyperreactivity. $J$. Immunol. 2010, 185, 5225-35.

160. Rodriguez-Manzanet, R.; Sanjuan, M.A.; Wu, H.Y.; Quintana, F.J.; Xiao, S.; Anderson, A.C.; Weiner, H.L.; Green, D.R.; Kuchroo, V.K. T and B cell hyperactivity and autoimmunity associated with niche-specific defects in apoptotic body clearance in TIM-4-deficient mice. Proc. Natl. Acad. Sci. U. S. A. 2010, 107, 8706-11.

161. Wong, K.; Valdez, P.A.; Tan, C.; Yeh, S.; Hongo, J.-A.; Ouyang, W. Phosphatidylserine receptor Tim-4 is essential for the maintenance of the homeostatic state of resident peritoneal macrophages. Proc. Natl. Acad. Sci. U. S. A. 2010, 107, 8712-7.

162. Kim, H.Y.; Chang, Y.-J.; Chuang, Y.-T.; Lee, H.-H.; Kasahara, D.I.; Martin, T.; Hsu, J.T.; Savage, P.B.; Shore, S.A.; Freeman, G.J.; et al. T-cell immunoglobulin and mucin domain 1 deficiency eliminates airway hyperreactivity triggered by the recognition of airway cell death. J. Allergy Clin. Immunol. 2013, 132, 414-25.e6.

163. Ichimura, T.; Bonventre, J. V.; Bailly, V.; Wei, H.; Hession, C.A.; Cate, R.L.; Sanicola, M. Kidney injury molecule-1 (KIM-1), a putative epithelial cell adhesion molecule containing a novel immunoglobulin domain, is up-regulated in renal cells after injury. J. Biol. Chem. 1998, 273, 4135-42.

164. Umetsu, S.E.; Lee, W.-L.; McIntire, J.J.; Downey, L.; Sanjanwala, B.; Akbari, O.; Berry, G.J.; Nagumo, H.; Freeman, G.J.; Umetsu, D.T.; et al. TIM-1 induces T cell activation and inhibits the development of peripheral tolerance. Nat. Immunol. 2005, 6, 447-54.

165. Sizing, I.D.; Bailly, V.; McCoon, P.; Chang, W.; Rao, S.; Pablo, L.; Rennard, R.; Walsh, M.; Li, Z.; Zafari, M.; et al. Epitope-dependent effect of anti-murine TIM1 monoclonal antibodies on $\mathrm{T}$ cell activity and lung immune responses. $J$. Immunol. 2007, 178, 2249-61.

166. Nakae, S.; likura, M.; Suto, H.; Akiba, H.; Umetsu, D.T.; Dekruyff, R.H.; Saito, H.; Galli, S.J. TIM-1 and TIM-3 enhancement of Th2 cytokine production by mast cells. Blood 2007, 110, 2565-8.

167. Kondratowicz, A.S.; Lennemann, N.J.; Sinn, P.L.; Davey, R.A.; Hunt, C.L.; Moller-Tank, S.; Meyerholz, D.K.; Rennert, P.; Mullins, R.F.; Brindley, M.; et al. T-cell immunoglobulin and mucin domain 1 (TIM-1) is a receptor for Zaire Ebolavirus and Lake Victoria Marburgvirus. Proc. Natl. Acad. Sci. 2011, 108, 8426-8431.

168. Meyers, J.H.; Chakravarti, S.; Schlesinger, D.; Illes, Z.; Waldner, H.; Umetsu, S.E.; Kenny, J.; Zheng, X.X.; Umetsu, D.T.; DeKruyff, R.H.; et al. TIM-4 is the ligand for TIM-1, and the TIM-1-TIM-4 interaction regulates T cell proliferation. Nat. Immunol. 2005, 6, 455-64.

169. Geisbert, T.W.; Hensley, L.E.; Larsen, T.; Young, H.A.; Reed, D.S.; Geisbert, J.B.; Scott, D.P.; Kagan, E.; Jahrling, P.B.; Davis, K.J. Pathogenesis of Ebola hemorrhagic fever in cynomolgus macaques: evidence that dendritic cells are early and sustained targets of infection. Am. J. Pathol. 2003, 163, 2347-70.

170. Martines, R.B.; Ng, D.L.; Greer, P.W.; Rollin, P.E.; Zaki, S.R. Tissue and cellular 
tropism, pathology and pathogenesis of Ebola and Marburg viruses. J. Pathol. 2015, 235, 153-174.

171. Stitt, T.N.; Conn, G.; Gore, M.; Lai, C.; Bruno, J.; Radziejewski, C.; Mattsson, K.; Fisher, J.; Gies, D.R.; Jones, P.F. The anticoagulation factor protein $S$ and its relative, Gas6, are ligands for the Tyro 3/Axl family of receptor tyrosine kinases. Cell 1995, 80, 661-70.

172. Godowski, P.J.; Mark, M.R.; Chen, J.; Sadick, M.D.; Raab, H.; Hammonds, R.G. Reevaluation of the roles of protein $S$ and Gas6 as ligands for the receptor tyrosine kinase Rse/Tyro 3. Cell 1995, 82, 355-8.

173. Lemke, G.; Rothlin, C. V. Immunobiology of the TAM receptors. Nat. Rev. Immunol. 2008, 8, 327-36.

174. Scott, R.S.; McMahon, E.J.; Pop, S.M.; Reap, E.A.; Caricchio, R.; Cohen, P.L.; Earp, H.S.; Matsushima, G.K. Phagocytosis and clearance of apoptotic cells is mediated by MER. Nature 2001, 411, 207-11.

175. Hafizi, S.; Dahlbäck, B. Gas6 and protein S. Vitamin K-dependent ligands for the AxI receptor tyrosine kinase subfamily. FEBS J. 2006, 273, 5231-44.

176. Rothlin, C. V.; Ghosh, S.; Zuniga, E.I.; Oldstone, M.B.A.; Lemke, G. TAM receptors are pleiotropic inhibitors of the innate immune response. Cell 2007, 131, 1124-36.

177. Bhattacharyya, S.; Zagórska, A.; Lew, E.D.; Shrestha, B.; Rothlin, C. V.; Naughton, J.; Diamond, M.S.; Lemke, G.; Young, J.A.T. Enveloped viruses disable innate immune responses in dendritic cells by direct activation of TAM receptors. Cell Host Microbe 2013, 14, 136-147.

178. Prieto, A.L.; Weber, J.L.; Lai, C. Expression of the receptor protein-tyrosine kinases Tyro-3, Axl, and mer in the developing rat central nervous system. J. Comp. Neurol. 2000, 425, 295-314.

179. Angelillo-Scherrer, A.; de Frutos, P.; Aparicio, C.; Melis, E.; Savi, P.; Lupu, F.; Arnout, J.; Dewerchin, M.; Hoylaerts, M.; Herbert, J.; et al. Deficiency or inhibition of Gas6 causes platelet dysfunction and protects mice against thrombosis. Nat. Med. 2001, 7, 215-21.

180. Caraux, A.; Lu, Q.; Fernandez, N.; Riou, S.; Di Santo, J.P.; Raulet, D.H.; Lemke, G.; Roth, C. Natural killer cell differentiation driven by Tyro3 receptor tyrosine kinases. Nat. Immunol. 2006, 7, 747-54.

181. Seitz, H.M.; Camenisch, T.D.; Lemke, G.; Earp, H.S.; Matsushima, G.K. Macrophages and dendritic cells use different Axl/Mertk/Tyro3 receptors in clearance of apoptotic cells. J. Immunol. 2007, 178, 5635-42.

182. Saeed, M.F.; Kolokoltsov, A.A.; Albrecht, T.; Davey, R.A. Cellular entry of ebola virus involves uptake by a macropinocytosis-like mechanism and subsequent trafficking through early and late endosomes. PLoS Pathog. 2010, 6, e1001110.

183. Brindley, M.A.; Hunt, C.L.; Kondratowicz, A.S.; Bowman, J.; Sinn, P.L.; McCray, P.B.; Quinn, K.; Weller, M.L.; Chiorini, J.A.; Maury, W. Tyrosine kinase receptor Axl enhances entry of Zaire ebolavirus without direct interactions with the viral glycoprotein. Virology 2011, 415, 83-94.

184. Hunt, C.L.; Kolokoltsov, A.A.; Davey, R.A.; Maury, W. The Tyro3 Receptor Kinase Axl Enhances Macropinocytosis of Zaire Ebolavirus. J. Virol. 2011, 85, 334-347.

185. Saeed, M.F.; Kolokoltsov, A.A.; Freiberg, A.N.; Holbrook, M.R.; Davey, R.A. Phosphoinositide-3 kinase-Akt pathway controls cellular entry of Ebola virus. PLoS Pathog. 2008, 4, e1000141.

186. Moller-Tank, S.; Albritton, L.M.; Rennert, P.D.; Maury, W. Characterizing 
functional domains for TIM-mediated enveloped virus entry. J. Virol. 2014, 88, 6702-13.

187. Nanbo, A.; Maruyama, J.; Imai, M.; Ujie, M.; Fujioka, Y.; Nishide, S.; Takada, A.; Ohba, Y.; Kawaoka, Y. Ebola virus requires a host scramblase for externalization of phosphatidylserine on the surface of viral particles. PLoS Pathog. 2018, 14, $1-25$.

188. Zelensky, A.N.; Gready, J.E. The C-type lectin-like domain superfamily. FEBS J. 2005, 272, 6179-217.

189. Mayer, S.; Raulf, M.K.; Lepenies, B. C-type lectins: their network and roles in pathogen recognition and immunity. Histochem. Cell Biol. 2017, 147, 223-237.

190. Mitchell, D.E.; Gibson, M.I. Latent Ice Recrystallization Inhibition Activity in Nonantifreeze Proteins: Ca2+-Activated Plant Lectins and Cation-Activated Antimicrobial Peptides. Biomacromolecules 2015, 16, 3411-6.

191. Robinson, M.J.; Sancho, D.; Slack, E.C.; LeibundGut-Landmann, S.; Reis e Sousa, C. Myeloid C-type lectins in innate immunity. Nat. Immunol. 2006, 7, 1258-65.

192. Dam, T.K.; Brewer, C.F. Lectins as pattern recognition molecules: the effects of epitope density in innate immunity. Glycobiology 2010, 20, 270-9.

193. Mason, C.P.; Tarr, A.W. Human lectins and their roles in viral infections. Molecules 2015, 20, 2229-2271.

194. Liu, Y.; Liu, J.; Pang, X.; Liu, T.; Ning, Z.; Cheng, G. The roles of direct recognition by animal lectins in antiviral immunity and viral pathogenesis. Molecules 2015, 20, 2272-95.

195. Geijtenbeek, T.B.H.; Torensma, R.; van Vliet, S.J.; van Duijnhoven, G.C.; Adema, G.J.; van Kooyk, Y.; Figdor, C.G. Identification of DC-SIGN, a novel dendritic cell-specific ICAM-3 receptor that supports primary immune responses. Cell 2000, 100, 575-85.

196. Mitchell, D.A.; Fadden, A.J.; Drickamer, K. A novel mechanism of carbohydrate recognition by the C-type lectins DC-SIGN and DC-SIGNR. Subunit organization and binding to multivalent ligands. J. Biol. Chem. 2001, 276, 28939-45.

197. van Liempt, E.; Bank, C.M.C.; Mehta, P.; Garciá-Vallejo, J.J.; Kawar, Z.S.; Geyer, R.; Alvarez, R.A.; Cummings, R.D.; Kooyk, Y. van; van Die, I. Specificity of DC-SIGN for mannose- and fucose-containing glycans. FEBS Lett. 2006, 580, 6123-31.

198. Khoo, U.-S.; Chan, K.Y.K.; Chan, V.S.F.; Lin, C.L.S. DC-SIGN and L-SIGN: the SIGNs for infection. J. Mol. Med. (Berl). 2008, 86, 861-74.

199. Lozach, P.-Y.; Burleigh, L.; Staropoli, I.; Amara, A. The C type lectins DC-SIGN and L-SIGN: receptors for viral glycoproteins. Methods Mol. Biol. 2007, 379, 5168.

200. Simmons, G.; Reeves, J.D.; Grogan, C.C.; Vandenberghe, L.H.; Baribaud, F.; Whitbeck, J.C.; Burke, E.; Buchmeier, M.J.; Soilleux, E.J.; Riley, J.L.; et al. DCSIGN and DC-SIGNR bind ebola glycoproteins and enhance infection of macrophages and endothelial cells. Virology 2003, 305, 115-23.

201. Brauburger, K.; Hume, A.J.; Mühlberger, E.; Olejnik, J. Forty-five years of Marburg virus research. Viruses 2012, 4, 1878-927.

202. da Silva, R.C.; Segat, L.; Crovella, S. Role of DC-SIGN and L-SIGN receptors in HIV-1 vertical transmission. Hum. Immunol. 2011, 72, 305-11.

203. Ludwig, I.S.; Lekkerkerker, A.N.; Depla, E.; Bosman, F.; Musters, R.J.P.; Depraetere, S.; van Kooyk, Y.; Geijtenbeek, T.B.H. Hepatitis C virus targets DCSIGN and L-SIGN to escape lysosomal degradation. J. Virol. 2004, 78, 8322- 
32.

204. Op den Brouw, M.L.; de Jong, M.A.W.P.; Ludwig, I.S.; van der Molen, R.G.; Janssen, H.L.A.; Geijtenbeek, T.B.H.; Woltman, A.M. Branched oligosaccharide structures on HBV prevent interaction with both DC-SIGN and L-SIGN. J. Viral Hepat. 2008, 15, 675-83.

205. Han, D.P.; Lohani, M.; Cho, M.W. Specific asparagine-linked glycosylation sites are critical for DC-SIGN- and L-SIGN-mediated severe acute respiratory syndrome coronavirus entry. J. Virol. 2007, 81, 12029-39.

206. Liu, W.; Tang, L.; Zhang, G.; Wei, H.; Cui, Y.; Guo, L.; Gou, Z.; Chen, X.; Jiang, D.; Zhu, Y.; et al. Characterization of a novel C-type lectin-like gene, LSECtin: demonstration of carbohydrate binding and expression in sinusoidal endothelial cells of liver and lymph node. J. Biol. Chem. 2004, 279, 18748-58.

207. Gramberg, T.; Hofmann, H.; Möller, P.; Lalor, P.F.; Marzi, A.; Geier, M.; Krumbiegel, M.; Winkler, T.; Kirchhoff, F.; Adams, D.H.; et al. LSECtin interacts with filovirus glycoproteins and the spike protein of SARS coronavirus. Virology 2005, 340, 224-236.

208. Gramberg, T.; Soilleux, E.; Fisch, T.; Lalor, P.F.; Hofmann, H.; Wheeldon, S.; Cotterill, A.; Wegele, A.; Winkler, T.; Adams, D.H.; et al. Interactions of LSECtin and DC-SIGN/DC-SIGNR with viral ligands: Differential $\mathrm{pH}$ dependence, internalization and virion binding. Virology 2008, 373, 189-201.

209. Takada, A.; Fujioka, K.; Tsuiji, M.; Morikawa, A.; Higashi, N.; Ebihara, H.; Kobasa, D.; Feldmann, H.; Irimura, T.; Kawaoka, Y. Human macrophage C-type lectin specific for galactose and $\mathrm{N}$-acetylgalactosamine promotes filovirus entry. J. Virol. 2004, 78, 2943-7.

210. Upham, J.P.; Pickett, D.; Irimura, T.; Anders, E.M.; Reading, P.C. Macrophage receptors for influenza $A$ virus: role of the macrophage galactose-type lectin and mannose receptor in viral entry. J. Virol. 2010, 84, 3730-7.

211. Usami, K.; Matsuno, K.; Igarashi, M.; Denda-Nagai, K.; Takada, A.; Irimura, T. Involvement of viral envelope GP2 in Ebola virus entry into cells expressing the macrophage galactose-type C-type lectin. Biochem. Biophys. Res. Commun. 2011, 407, 74-8.

212. Ng, W.C.; Liong, S.; Tate, M.D.; Irimura, T.; Denda-Nagai, K.; Brooks, A.G.; Londrigan, S.L.; Reading, P.C. The macrophage galactose-type lectin can function as an attachment and entry receptor for influenza virus. J. Virol. 2014, 88, 1659-72.

213. Zizzari, I.G.; Napoletano, C.; Battisti, F.; Rahimi, H.; Caponnetto, S.; Pierelli, L.; Nuti, M.; Rughetti, A. MGL Receptor and Immunity: When the Ligand Can Make the Difference. J. Immunol. Res. 2015, 2015, 450695.

214. Becker, S.; Spiess, M.; Klenk, H.D. The asialoglycoprotein receptor is a potential liver-specific receptor for Marburg virus. J. Gen. Virol. 1995, 76 ( Pt 2), 393-9.

215. Cummings, R.D.; McEver, R.P. C-type Lectins; 2009; ISBN 9780879697709.

216. Alvarez, C.P.; Lasala, F.; Carrillo, J.; Muñiz, O.; Corbí, A.L.; Delgado, R. C-Type Lectins DC-SIGN and L-SIGN Mediate Cellular Entry by Ebola Virus in cis and in trans. J. Virol. 2002, 76, 6841-6844.

217. Marzi, A.; Gramberg, T.; Simmons, G.; Möller, P.; Rennekamp, A.J.; Krumbiegel, M.; Geier, M.; Eisemann, J.; Turza, N.; Saunier, B.; et al. DC-SIGN and DCSIGNR interact with the glycoprotein of Marburg virus and the S protein of severe acute respiratory syndrome coronavirus. J. Virol. 2004, 78, 12090-5.

218. Marzi, A.; Möller, P.; Hanna, S.L.; Harrer, T.; Eisemann, J.; Steinkasserer, A.; Becker, S.; Baribaud, F.; Pöhlmann, S. Analysis of the Interaction of Ebola Virus 
Glycoprotein with DC-SIGN (Dendritic Cell-Specific Intercellular Adhesion Molecule 3-Grabbing Nonintegrin) and Its Homologue DC-SIGNR. J. Infect. Dis. 2007, 196, S237-S246.

219. Matsuno, K.; Nakayama, E.; Noyori, O.; Marzi, A.; Ebihara, H.; Irimura, T.; Feldmann, H.; Takada, A. C-type lectins do not act as functional receptors for filovirus entry into cells. Biochem. Biophys. Res. Commun. 2010, 403, 144-8.

220. Sanchez, A. Analysis of Filovirus Entry into Vero E6 Cells, Using Inhibitors of Endocytosis, Endosomal Acidification, Structural Integrity, and Cathepsin (B and L) Activity. J. Infect. Dis. 2007, 196, S251-S258.

221. Bhattacharyya, S.; Warfield, K.L.; Ruthel, G.; Bavari, S.; Aman, M.J.; Hope, T.J. Ebola virus uses clathrin-mediated endocytosis as an entry pathway. Virology 2010, 401, 18-28.

222. Bhattacharyya, S.; Hope, T.J.; Young, J.A.T. Differential requirements for clathrin endocytic pathway components in cellular entry by Ebola and Marburg glycoprotein pseudovirions. Virology 2011, 419, 1-9.

223. Nanbo, A.; Imai, M.; Watanabe, S.; Noda, T.; Takahashi, K.; Neumann, G.; Halfmann, P.; Kawaoka, Y. Ebolavirus is internalized into host cells via macropinocytosis in a viral glycoprotein-dependent manner. PLoS Pathog. 2010, 6, e1001121.

224. Krysko, D. V.; Denecker, G.; Festjens, N.; Gabriels, S.; Parthoens, E.; D'Herde, K.; Vandenabeele, P. Macrophages use different internalization mechanisms to clear apoptotic and necrotic cells. Cell Death Differ. 2006.

225. Sallusto, F. Dendritic cells use macropinocytosis and the mannose receptor to concentrate macromolecules in the major histocompatibility complex class II compartment: downregulation by cytokines and bacterial products. J. Exp. Med. 1995.

226. Rossi, A.; Deveraux, Q.; Turk, B.; Sali, A. Comprehensive search for cysteine cathepsins in the human genome. Biol. Chem. 2004, 385, 363-372.

227. Turk, V.; Stoka, V.; Vasiljeva, O.; Renko, M.; Sun, T.; Turk, B.; Turk, D. Cysteine cathepsins: From structure, function and regulation to new frontiers. Biochim. Biophys. Acta - Proteins Proteomics 2012, 1824, 68-88.

228. Rawlings, N.D.; Barrett, A.J.; Thomas, P.D.; Huang, X.; Bateman, A.; Finn, R.D. The MEROPS database of proteolytic enzymes, their substrates and inhibitors in 2017 and a comparison with peptidases in the PANTHER database. Nucleic Acids Res. 2018, 46, D624-D632.

229. Brix, K.; Dunkhorst, A.; Mayer, K.; Jordans, S. Cysteine cathepsins: Cellular roadmap to different functions. Biochimie 2008, 90, 194-207.

230. Turk, B.; Turk, D.; Turk, V. Lysosomal cysteine proteases: More than scavengers. Biochim. Biophys. Acta - Protein Struct. Mol. Enzymol. 2000, 1477, 98-111.

231. Brix, K.; McInnes, J.; Al-Hashimi, A.; Rehders, M.; Tamhane, T.; Haugen, M.H. Proteolysis mediated by cysteine cathepsins and legumain-recent advances and cell biological challenges. Protoplasma 2015, 252, 755-74.

232. Erickson, A.H.; Isidoro, C.; Mach, L.; Mort, J.S. Cathepsins: Getting in shape for lysosomal proteolysis. In Proteases: Structure and Function; 2013; pp. 127-73 ISBN 9783709108857.

233. Musil, D.; Zucic, D.; Turk, D.; Engh, R.A.; Mayr, I.; Huber, R.; Popovic, T.; Turk, V.; Towatari, T.; Katunuma, N.; et al. The refined $2.15 \mathrm{~A} X$-ray crystal structure of human liver cathepsin B: the structural basis for its specificity. EMBO J.1991, $10,2321-2330$. 
234. Brix, K. Host Cell Proteases: Cathepsins. In Activation of Viruses by Host Proteases; Böttcher-Friebertshäuser, E., Garten, W., Klenk, H., Eds.; Springer, Cham, 2018; pp. 249-276 ISBN 978-3-319-75473-4.

235. Turk, V.; Turk, B.; Turk, D. Lysosomal cysteine proteases: Facts and opportunities. EMBO J. 2001, 20, 4629-4633.

236. Reiser, J.; Adair, B.; Reinheckel, T. Specialized roles for cysteine cathepsins in health and disease. J. Clin. Invest. 2010, 120, 3421-31.

237. Gansz, M.; Kern, U.; Peters, C.; Reinheckel, T. Exploring systemic functions of lysosomal proteases: The perspective of genetically modified mouse models. In Proteases: Structure and Function; 2013; pp. 217-33 ISBN 9783709108857.

238. Sloane, B.F.; List, K.; Fingleton, B.; Matrisian, L. Proteases in cancer: Significance for invasion and metastasis. In Proteases: Structure and Function; 2013; p. TBD ISBN 9783709108857.

239. Kirschke, H. Chapter 410 - Cathepsin L. In Handbook of Proteolytic Enzymes (Third Edition); Rawlings, N.D., Salvesen, G., Eds.; Academic Press, 2013; pp. 1808-1817 ISBN 978-0-12-382219-2.

240. Mort, J.S. Chapter 406 - Cathepsin B. In Handbook of Proteolytic Enzymes (Third Edition); Rawlings, N.D., Salvesen, G., Eds.; Academic Press, 2013; pp. 1784-1791 ISBN 978-0-12-382219-2.

241. Chandran, K.; Sullivan, N.J.; Felbor, U.; Whelan, S.P.; Cunningham, J.M. Endosomal proteolysis of the ebola virus glycoprotein is necessary for infection. Science (80-. ). 2005, 308, 1643-1645.

242. Schornberg, K.; Matsuyama, S.; Kabsch, K.; Delos, S.; Bouton, A.; White, J. Role of Endosomal Cathepsins in Entry Mediated by the Ebola Virus Glycoprotein. J. Virol. 2006, 80, 4174-4178.

243. Kaletsky, R.L.; Simmons, G.; Bates, P. Proteolysis of the Ebola Virus Glycoproteins Enhances Virus Binding and Infectivity. J. Virol. 2007, 81, 1337813384.

244. Dube, D.; Brecher, M.B.; Delos, S.E.; Rose, S.C.; Park, E.W.; Schornberg, K.L.; Kuhn, J.H.; White, J.M. The Primed Ebolavirus Glycoprotein (19-Kilodalton GP1,2): Sequence and Residues Critical for Host Cell Binding. J. Virol. 2009, 83, 2883-91.

245. Hood, C.L.; Abraham, J.; Boyington, J.C.; Leung, K.; Kwong, P.D.; Nabel, G.J. Biochemical and Structural Characterization of Cathepsin L-Processed Ebola Virus Glycoprotein: Implications for Viral Entry and Immunogenicity. J. Virol. 2010, 84, 2972-2982.

246. Volchkov, V.; Klenk, H.D. Proteolytic Processing of Filovirus Glycoproteins. In Activation of Viruses by Host Proteases; Böttcher-Friebertshäuser, E., Garten, W., Klenk, H., Eds.; Springer, Cham, 2018; pp. 99-108 ISBN 978-3-319-754734.

247. Misasi, J.; Chandran, K.; Yang, J.-Y.; Considine, B.; Filone, C.M.; Cote, M.; Sullivan, N.; Fabozzi, G.; Hensley, L.; Cunningham, J. Filoviruses Require Endosomal Cysteine Proteases for Entry but Exhibit Distinct Protease Preferences. J. Virol. 2012, 86, 3284-92.

248. Hoffmann, M.; González Hernández, M.; Berger, E.; Marzi, A.; Pöhlmann, S. The Glycoproteins of All Filovirus Species Use the Same Host Factors for Entry into Bat and Human Cells but Entry Efficiency Is Species Dependent. PLoS One 2016, 11, e0149651.

249. Hofmann-Winkler, H.; Gnirß, K.; Wrensch, F.; Pöhlmann, S. Comparative Analysis of Host Cell Entry of Ebola Virus from Sierra Leone, 2014, and Zaire, 
1976. J. Infect. Dis. 2015, 212, S172-S180.

250. Gnirß, K.; Kühl, A.; Karsten, C.; Glowacka, I.; Bertram, S.; Kaup, F.; Hofmann, H.; Pöhlmann, S. Cathepsins B and L activate Ebola but not Marburg virus glycoproteins for efficient entry into cell lines and macrophages independent of TMPRSS2 expression. Virology 2012, 424, 3-10.

251. Marzi, A.; Reinheckel, T.; Feldmann, H. Cathepsin B \& L are not required for ebola virus replication. PLoS Negl. Trop. Dis. 2012, 6, e1923.

252. Carstea, E.D.; Morris, J.A.; Coleman, K.G.; Loftus, S.K.; Zhang, D.; Cummings, C.; Gu, J.; Rosenfeld, M.A.; Pavan, W.J.; Krizman, D.B.; et al. Niemann-Pick C1 disease gene: homology to mediators of cholesterol homeostasis. Science 1997, 277, 228-31.

253. Davies, J.P.; loannou, Y.A. Topological analysis of Niemann-Pick C1 protein reveals that the membrane orientation of the putative sterol-sensing domain is identical to those of 3-hydroxy-3-methylglutaryl-CoA reductase and sterol regulatory element binding protein cleavage-activating. J. Biol. Chem. 2000, 275, 24367-74.

254. Kwon, H.J.; Abi-Mosleh, L.; Wang, M.L.; Deisenhofer, J.; Goldstein, J.L.; Brown, M.S.; Infante, R.E. Structure of N-terminal domain of NPC1 reveals distinct subdomains for binding and transfer of cholesterol. Cell 2009, 137, 1213-24.

255. Wang, H.; Shi, Y.; Song, J.; Qi, J.; Lu, G.; Yan, J.; Gao, G.F. Ebola Viral Glycoprotein Bound to Its Endosomal Receptor Niemann-Pick C1. Cell 2016, 164, 258-268.

256. Cruz, J.C.; Sugii, S.; Yu, C.; Chang, T.Y. Role of Niemann-Pick type C1 protein in intracellular trafficking of low density lipoprotein-derived cholesterol. J. Biol. Chem. 2000, 275, 4013-21.

257. Sleat, D.E.; Wiseman, J.A.; El-Banna, M.; Price, S.M.; Verot, L.; Shen, M.M.; Tint, G.S.; Vanier, M.T.; Walkley, S.U.; Lobel, P. Genetic evidence for nonredundant functional cooperativity between NPC1 and NPC2 in lipid transport. Proc. Natt. Acad. Sci. U. S. A. 2004, 101, 5886-91.

258. Infante, R.E.; Wang, M.L.; Radhakrishnan, A.; Kwon, H.J.; Brown, M.S.; Goldstein, J.L. NPC2 facilitates bidirectional transfer of cholesterol between NPC1 and lipid bilayers, a step in cholesterol egress from lysosomes. Proc. Natl. Acad. Sci. U. S. A. 2008, 105, 15287-92.

259. Wang, M.L.; Motamed, M.; Infante, R.E.; Abi-Mosleh, L.; Kwon, H.J.; Brown, M.S.; Goldstein, J.L. Identification of surface residues on Niemann-Pick C2 essential for hydrophobic handoff of cholesterol to NPC1 in lysosomes. Cell Metab. 2010, 12, 166-73.

260. Pentchev, P.G. Niemann-Pick C research from mouse to gene. Biochim. Biophys. Acta 2004, 1685, 3-7.

261. Dixit, S.S.; Jadot, M.; Sohar, I.; Sleat, D.E.; Stock, A.M.; Lobel, P. Loss of Niemann-Pick $\mathrm{C} 1$ or $\mathrm{C} 2$ protein results in similar biochemical changes suggesting that these proteins function in a common lysosomal pathway. PLoS One 2011, 6, e23677.

262. Rosenbaum, A.I.; Maxfield, F.R. Niemann-Pick type C disease: molecular mechanisms and potential therapeutic approaches. J. Neurochem. 2011, 116, 789-95.

263. Shoemaker, C.J.; Schornberg, K.L.; Delos, S.E.; Scully, C.; Pajouhesh, H.; Olinger, G.G.; Johansen, L.M.; White, J.M. Multiple cationic amphiphiles induce a Niemann-Pick $C$ phenotype and inhibit Ebola virus entry and infection. PLoS One 2013, 8, e56265. 
264. Miller, E.H.; Obernosterer, G.; Raaben, M.; Herbert, A.S.; Deffieu, M.S.; Krishnan, A.; Ndungo, E.; Sandesara, R.G.; Carette, J.E.; Kuehne, A.I.; et al. Ebola virus entry requires the host-programmed recognition of an intracellular receptor. EMBO J. 2012, 31, 1947-1960.

265. Lu, F.; Liang, Q.; Abi-Mosleh, L.; Das, A.; De Brabander, J.K.; Goldstein, J.L.; Brown, M.S. Identification of NPC1 as the target of U18666A, an inhibitor of lysosomal cholesterol export and Ebola infection. Elife 2015, 4.

266. Bornholdt, Z.A.; Ndungo, E.; Fusco, M.L.; Bale, S.; Flyak, A.I.; Crowe, J.E.; Chandran, K.; Saphire, E.O. Host-primed Ebola virus GP exposes a hydrophobic NPC1 receptor-binding pocket, revealing a target for broadly neutralizing antibodies. MBio 2016, 7, 1-11.

267. Gong, X.; Qian, H.; Zhou, X.; Wu, J.; Wan, T.; Cao, P.; Huang, W.; Zhao, X.; Wang, X.; Wang, P.; et al. Structural Insights into the Niemann-Pick C1 (NPC1)Mediated Cholesterol Transfer and Ebola Infection. Cell 2016, 165, 1467-1478.

268. Herbert, A.S.; Davidson, C.; Kuehne, A.I.; Bakken, R.; Braigen, S.Z.; Gunn, K.E.; Whelan, S.P.; Brummelkamp, T.R.; Twenhafel, N. a; Chandran, K.; et al. Niemann-pick $\mathrm{C} 1$ is essential for ebolavirus replication and pathogenesis in vivo. MBio 2015, 6, e00565-15.

269. Kuroda, M.; Fujikura, D.; Nanbo, A.; Marzi, A.; Noyori, O.; Kajihara, M.; Maruyama, J.; Matsuno, K.; Miyamoto, H.; Yoshida, R.; et al. Interaction between TIM-1 and NPC1 Is Important for Cellular Entry of Ebola Virus. J. Virol. 2015, 89, 6481-6493.

270. Alberts, B.; Johnson, A.; Lewis, J.; Raff, M.; Roberts, K.; Walter, P. Pathogens, Infection, and Innate Immunity. In Molecular biology of the cell; Anderson, M., Granum, S., Eds.; Garland science, Taylor \& Francis Group, LLC, 2008; pp. 1485-1538.

271. Owen, J.A.; Punt, J.; Stranford, S.A.; Jones, P.P.; Kuby, J. Chapter 1: Overview of the Immune System. In Kuby immunology 7th Edition; Macmillan Higher Education, 2013.

272. Owen, J.A.; Punt, J.; Stranford, S.A.; Jones, P.P.; Kuby, J. Chapter 5: Innate Immunity. In Kuby immunology 7th Edition; Macmillan Higher Education, 2013 ISBN $9781429219198142921919 X 97814641378461464137846$.

273. Iwasaki, A.; Medzhitov, R. Control of adaptive immunity by the innate immune system. Nat. Immunol. 2015, 16, 343-53.

274. Brehélin, M.; Roch, P. Specificity, learning and memory in the innate immune response. ISJ Vis. Perspect 2008, 5, 103-9.

275. Netea, M.G. Training innate immunity: the changing concept of immunological memory in innate host defence. Eur. J. Clin. Invest. 2013, 43, 881-4.

276. Netea, M.G.; Latz, E.; Mills, K.H.G.; O'Neill, L.A.J. Innate immune memory: a paradigm shift in understanding host defense. Nat. Immunol. 2015, 16, 675-9.

277. Riera Romo, M.; Pérez-Martínez, D.; Castillo Ferrer, C. Innate immunity in vertebrates: An overview. Immunology 2016, 148, 125-139.

278. Ip, W.K.E.; Takahashi, K.; Ezekowitz, R.A.; Stuart, L.M. Mannose-binding lectin and innate immunity. Immunol. Rev. 2009, 230, 9-21.

279. Newton, K.; Dixit, V.M. Signaling in innate immunity and inflammation. Cold Spring Harb. Perspect. Biol. 2012, 4.

280. Müller, U.; Steinhoff, U.; Reis, L.F.L.; Hemmi, S.; Pavlovic, J.; Zinkernagel, R.M.; Aguet, M. Functional role of type I and type II interferons in antiviral defense. Science 1994, 264, 1918-21.

281. van Pesch, V.; Lanaya, H.; Renauld, J.-C.; Michiels, T. Characterization of the 
murine alpha interferon gene family. J. Virol. 2004, 78, 8219-28.

282. Kotenko, S. V.; Gallagher, G.; Baurin, V. V.; Lewis-Antes, A.; Shen, M.; Shah, N.K.; Langer, J.A.; Sheikh, F.; Dickensheets, H.; Donnelly, R.P. IFN-lambdas mediate antiviral protection through a distinct class II cytokine receptor complex. Nat. Immunol. 2003, 4, 69-77.

283. Sheppard, P.; Kindsvogel, W.; Xu, W.; Henderson, K.; Schlutsmeyer, S.; Whitmore, T.E.; Kuestner, R.; Garrigues, U.; Birks, C.; Roraback, J.; et al. IL-28, IL-29 and their class II cytokine receptor IL-28R. Nat. Immunol. 2003, 4, 63-8.

284. Prokunina-Olsson, L.; Muchmore, B.; Tang, W.; Pfeiffer, R.M.; Park, H.; Dickensheets, H.; Hergott, D.; Porter-Gill, P.; Mumy, A.; Kohaar, I.; et al. A variant upstream of IFNL3 (IL28B) creating a new interferon gene IFNL4 is associated with impaired clearance of hepatitis C virus. Nat. Genet. 2013, 45, 164-71.

285. Chow, K.T.; Gale, M. SnapShot: Interferon Signaling. Cell 2015, 163, 18081808.e1.

286. Thompson, M.R.; Kaminski, J.J.; Kurt-Jones, E.A.; Fitzgerald, K.A. Pattern recognition receptors and the innate immune response to viral infection. Viruses 2011, 3, 920-40.

287. Weber, M.; Gawanbacht, A.; Habjan, M.; Rang, A.; Borner, C.; Schmidt, A.M.; Veitinger, S.; Jacob, R.; Devignot, S.; Kochs, G.; et al. Incoming RNA virus nucleocapsids containing a 5'-triphosphorylated genome activate RIG-I and antiviral signaling. Cell Host Microbe 2013, 13, 336-46.

288. Goubau, D.; Schlee, M.; Deddouche, S.; Pruijssers, A.J.; Zillinger, T.; Goldeck, M.; Schuberth, C.; Van der Veen, A.G.; Fujimura, T.; Rehwinkel, J.; et al. Antiviral immunity via RIG-I-mediated recognition of RNA bearing 5'-diphosphates. Nature 2014, 514, 372-375.

289. Yoneyama, M.; Onomoto, K.; Jogi, M.; Akaboshi, T.; Fujita, T. Viral RNA detection by RIG-I-like receptors. Curr. Opin. Immunol. 2015, 32, 48-53.

290. Kato, H.; Takeuchi, O.; Mikamo-Satoh, E.; Hirai, R.; Kawai, T.; Matsushita, K.; Hiiragi, A.; Dermody, T.S.; Fujita, T.; Akira, S. Length-dependent recognition of double-stranded ribonucleic acids by retinoic acid-inducible gene-I and melanoma differentiation-associated gene 5. J. Exp. Med. 2008, 205, 1601-10.

291. Züst, R.; Cervantes-Barragan, L.; Habjan, M.; Maier, R.; Neuman, B.W.; Ziebuhr, J.; Szretter, K.J.; Baker, S.C.; Barchet, W.; Diamond, M.S.; et al. Ribose 2'-Omethylation provides a molecular signature for the distinction of self and non-self mRNA dependent on the RNA sensor Mda5. Nat. Immunol. 2011, 12, 137-43.

292. Saito, T.; Hirai, R.; Loo, Y.-M.; Owen, D.; Johnson, C.L.; Sinha, S.C.; Akira, S.; Fujita, T.; Gale, M. Regulation of innate antiviral defenses through a shared repressor domain in RIG-I and LGP2. Proc. Natl. Acad. Sci. U. S. A. 2007, 104, 582-7.

293. Childs, K.S.; Randall, R.E.; Goodbourn, S. LGP2 plays a critical role in sensitizing mda-5 to activation by double-stranded RNA. PLoS One 2013, 8, e64202.

294. Bruns, A.M.; Leser, G.P.; Lamb, R.A.; Horvath, C.M. The innate immune sensor LGP2 activates antiviral signaling by regulating MDA5-RNA interaction and filament assembly. Mol. Cell 2014, 55, 771-81.

295. Di Virgilio, F. The therapeutic potential of modifying inflammasomes and NODlike receptors. Pharmacol. Rev. 2013, 65, 872-905.

296. Caruso, R.; Warner, N.; Inohara, N.; Núñez, G. NOD1 and NOD2: signaling, host defense, and inflammatory disease. Immunity 2014, 41, 898-908. 
297. Onoguchi, K.; Yoneyama, M.; Takemura, A.; Akira, S.; Taniguchi, T.; Namiki, H.; Fujita, T. Viral infections activate types I and III interferon genes through a common mechanism. J. Biol. Chem. 2007, 282, 7576-81.

298. Andersen, J.; VanScoy, S.; Cheng, T.-F.; Gomez, D.; Reich, N.C. IRF-3dependent and augmented target genes during viral infection. Genes Immun. 2008, 9, 168-75.

299. Ford, E.; Thanos, D. The transcriptional code of human IFN-beta gene expression. Biochim. Biophys. Acta 2010, 1799, 328-36.

300. Schoggins, J.W.; Wilson, S.J.; Panis, M.; Murphy, M.Y.; Jones, C.T.; Bieniasz, P.; Rice, C.M. A diverse range of gene products are effectors of the type I interferon antiviral response. Nature 2011, 472, 481-5.

301. Schneider, W.M.; Chevillotte, M.D.; Rice, C.M. Interferon-stimulated genes: a complex web of host defenses. Annu. Rev. Immunol. 2014, 32, 513-45.

302. Heaton, S.M.; Borg, N.A.; Dixit, V.M. Ubiquitin in the activation and attenuation of innate antiviral immunity. J. Exp. Med. 2016, 213, 1-13.

303. de Weerd, N.A.; Nguyen, T. The interferons and their receptors--distribution and regulation. Immunol. Cell Biol. 2012, 90, 483-91.

304. de Veer, M.J.; Holko, M.; Frevel, M.; Walker, E.; Der, S.; Paranjape, J.M.; Silverman, R.H.; Williams, B.R. Functional classification of interferon-stimulated genes identified using microarrays. J. Leukoc. Biol. 2001, 69, 912-20.

305. Raftery, N.; Stevenson, N.J. Advances in anti-viral immune defence: revealing the importance of the IFN JAK/STAT pathway. Cell. Mol. Life Sci. 2017, 74, 2525-2535.

306. Schoggins, J.W.; Rice, C.M. Interferon-stimulated genes and their antiviral effector functions. Curr. Opin. Virol. 2011, 1, 519-25.

307. Klockow, B.; Tichelaar, W.; Madden, D.R.; Niemann, H.H.; Akiba, T.; Hirose, K.; Manstein, D.J. The dynamin A ring complex: molecular organization and nucleotide-dependent conformational changes. EMBO J. 2002, 21, 240-50.

308. Gao, S.; von der Malsburg, A.; Paeschke, S.; Behlke, J.; Haller, O.; Kochs, G.; Daumke, $O$. Structural basis of oligomerization in the stalk region of dynaminlike MxA. Nature 2010, 465, 502-6.

309. Haller, O.; Gao, S.; von der Malsburg, A.; Daumke, O.; Kochs, G. Dynamin-like MxA GTPase: structural insights into oligomerization and implications for antiviral activity. J. Biol. Chem. 2010, 285, 28419-24.

310. Haller, O.; Staeheli, P.; Schwemmle, M.; Kochs, G. Mx GTPases: Dynamin-like antiviral machines of innate immunity. Trends Microbiol. 2015, 23, 154-163.

311. Schwemmle, M.; Weining, K.C.; Richter, M.F.; Schumacher, B.; Staeheli, P. Vesicular stomatitis virus transcription inhibited by purified MxA protein. Virology 1995, 206, 545-54.

312. Zhao, H.; De, B.P.; Das, T.; Banerjee, A.K. Inhibition of human parainfluenza virus-3 replication by interferon and human MxA. Virology 1996, 220, 330-8.

313. Kochs, G.; Haller, O. Interferon-induced human MxA GTPase blocks nuclear import of Thogoto virus nucleocapsids. Proc. Natl. Acad. Sci. U. S. A. 1999, 96, 2082-6.

314. Xiao, H.; Killip, M.J.; Staeheli, P.; Randall, R.E.; Jackson, D. The human interferon-induced MxA protein inhibits early stages of influenza $A$ virus infection by retaining the incoming viral genome in the cytoplasm. J. Virol. 2013, 87, 13053-8.

315. Goujon, C.; Moncorgé, O.; Bauby, H.; Doyle, T.; Ward, C.C.; Schaller, T.; Hué, S.; Barclay, W.S.; Schulz, R.; Malim, M.H. Human MX2 is an interferon-induced 
post-entry inhibitor of HIV-1 infection. Nature 2013, 502, 559-62.

316. Kane, M.; Yadav, S.S.; Bitzegeio, J.; Kutluay, S.B.; Zang, T.; Wilson, S.J.; Schoggins, J.W.; Rice, C.M.; Yamashita, M.; Hatziioannou, T.; et al. MX2 is an interferon-induced inhibitor of HIV-1 infection. Nature 2013, 502, 563-6.

317. Liu, Z.; Pan, Q.; Ding, S.; Qian, J.; Xu, F.; Zhou, J.; Cen, S.; Guo, F.; Liang, C. The interferon-inducible MxB protein inhibits HIV-1 infection. Cell Host Microbe 2013, 14, 398-410.

318. Fricke, T.; White, T.E.; Schulte, B.; de Souza Aranha Vieira, D.A.; Dharan, A.; Campbell, E.M.; Brandariz-Nuñez, A.; Diaz-Griffero, F. MxB binds to the HIV-1 core and prevents the uncoating process of HIV-1. Retrovirology 2014, 11, 68.

319. Friedman, R.L.; Manly, S.P.; McMahon, M.; Kerr, I.M.; Stark, G.R. Transcriptional and posttranscriptional regulation of interferon-induced gene expression in human cells. Cell 1984, 38, 745-55.

320. Perreira, J.M.; Chin, C.R.; Feeley, E.M.; Brass, A.L. IFITMs restrict the replication of multiple pathogenic viruses. J. Mol. Biol. 2013, 425, 4937-55.

321. Huang, I.-C.; Bailey, C.C.; Weyer, J.L.; Radoshitzky, S.R.; Becker, M.M.; Chiang, J.J.; Brass, A.L.; Ahmed, A.A.; Chi, X.; Dong, L.; et al. Distinct patterns of IFITMmediated restriction of filoviruses, SARS coronavirus, and influenza A virus. PLoS Pathog. 2011, 7, e1001258.

322. Feeley, E.M.; Sims, J.S.; John, S.P.; Chin, C.R.; Pertel, T.; Chen, L.-M.; Gaiha, G.D.; Ryan, B.J.; Donis, R.O.; Elledge, S.J.; et al. IFITM3 inhibits influenza A virus infection by preventing cytosolic entry. PLoS Pathog. 2011, 7, e1002337.

323. Diamond, M.S.; Farzan, M. The broad-spectrum antiviral functions of IFIT and IFITM proteins. Nat. Rev. Immunol. 2013, 13, 46-57.

324. Lu, J.; Pan, Q.; Rong, L.; He, W.; Liu, S.-L.; Liang, C. The IFITM proteins inhibit HIV-1 infection. J. Virol. 2011, 85, 2126-37.

325. Wilkins, C.; Woodward, J.; Lau, D.T.-Y.; Barnes, A.; Joyce, M.; McFarlane, N.; McKeating, J.A.; Tyrrell, D.L.; Gale, M. IFITM1 is a tight junction protein that inhibits hepatitis $C$ virus entry. Hepatology 2013, 57, 461-9.

326. Bailey, C.C.; Kondur, H.R.; Huang, I.-C.; Farzan, M. Interferon-induced transmembrane protein 3 is a type II transmembrane protein. J. Biol. Chem. 2013, 288, 32184-93.

327. Bailey, C.C.; Zhong, G.; Huang, I.-C.; Farzan, M. IFITM-Family Proteins: The Cell's First Line of Antiviral Defense. Annu. Rev. Virol. 2014, 1, 261-283.

328. Li, K.; Markosyan, R.M.; Zheng, Y.-M.; Golfetto, O.; Bungart, B.; Li, M.; Ding, S.; He, Y.; Liang, C.; Lee, J.C.; et al. IFITM proteins restrict viral membrane hemifusion. PLoS Pathog. 2013, 9, e1003124.

329. Ozato, K.; Shin, D.-M.; Chang, T.-H.; Morse, H.C. TRIM family proteins and their emerging roles in innate immunity. Nat. Rev. Immunol. 2008, 8, 849-60.

330. van Tol, S.; Hage, A.; Giraldo, M.; Bharaj, P.; Rajsbaum, R. The TRIMendous Role of TRIMs in Virus-Host Interactions. Vaccines 2017, 5, 23.

331. Stremlau, M.; Owens, C.M.; Perron, M.J.; Kiessling, M.; Autissier, P.; Sodroski, J. The cytoplasmic body component TRIM5alpha restricts HIV-1 infection in Old World monkeys. Nature 2004, 427, 848-53.

332. Barr, S.D.; Smiley, J.R.; Bushman, F.D. The interferon response inhibits HIV particle production by induction of TRIM22. PLoS Pathog. 2008, 4, e1000007.

333. Gao, B.; Duan, Z.; Xu, W.; Xiong, S. Tripartite motif-containing 22 inhibits the activity of hepatitis $B$ virus core promoter, which is dependent on nuclear-located RING domain. Hepatology 2009, 50, 424-33.

334. Eldin, P.; Papon, L.; Oteiza, A.; Brocchi, E.; Lawson, T.G.; Mechti, N. TRIM22 
E3 ubiquitin ligase activity is required to mediate antiviral activity against encephalomyocarditis virus. J. Gen. Virol. 2009, 90, 536-45.

335. Di Pietro, A.; Kajaste-Rudnitski, A.; Oteiza, A.; Nicora, L.; Towers, G.J.; Mechti, N.; Vicenzi, E. TRIM22 inhibits influenza A virus infection by targeting the viral nucleoprotein for degradation. J. Virol. 2013, 87, 4523-33.

336. Hovanessian, A.G.; Justesen, J. The human 2'-5'oligoadenylate synthetase family: unique interferon-inducible enzymes catalyzing 2'-5' instead of 3'-5' phosphodiester bond formation. Biochimie 2007, 89, 779-88.

337. Clemens, M.J.; Vaquero, C.M. Inhibition of protein synthesis by double-stranded RNA in reticulocyte lysates: evidence for activation of an endoribonuclease. Biochem. Biophys. Res. Commun. 1978, 83, 59-68.

338. Chakrabarti, A.; Jha, B.K.; Silverman, R.H. New insights into the role of RNase $\mathrm{L}$ in innate immunity. J. Interferon Cytokine Res. 2011, 31, 49-57.

339. Sadler, A.J.; Williams, B.R.G. Interferon-inducible antiviral effectors. Nat. Rev. Immunol. 2008, 8, 559-68.

340. Roberts, W.K.; Hovanessian, A.; Brown, R.E.; Clemens, M.J.; Kerr, I.M. Interferon-mediated protein kinase and low-molecular-weight inhibitor of protein synthesis. Nature 1976, 264, 477-80.

341. Pindel, A.; Sadler, A. The role of protein kinase $R$ in the interferon response. J. Interferon Cytokine Res. 2011, 31, 59-70.

342. Wang, X.; Hinson, E.R.; Cresswell, P. The interferon-inducible protein viperin inhibits influenza virus release by perturbing lipid rafts. Cell Host Microbe 2007, 2, 96-105.

343. Szretter, K.J.; Brien, J.D.; Thackray, L.B.; Virgin, H.W.; Cresswell, P.; Diamond, M.S. The interferon-inducible gene viperin restricts West Nile virus pathogenesis. J. Virol. 2011, 85, 11557-66.

344. Nasr, N.; Maddocks, S.; Turville, S.G.; Harman, A.N.; Woolger, N.; Helbig, K.J.; Wilkinson, J.; Bye, C.R.; Wright, T.K.; Rambukwelle, D.; et al. HIV-1 infection of human macrophages directly induces viperin which inhibits viral production. Blood 2012, 120, 778-88.

345. Helbig, K.J.; Eyre, N.S.; Yip, E.; Narayana, S.; Li, K.; Fiches, G.; McCartney, E.M.; Jangra, R.K.; Lemon, S.M.; Beard, M.R. The antiviral protein viperin inhibits hepatitis $C$ virus replication via interaction with nonstructural protein $5 \mathrm{~A}$. Hepatology 2011, 54, 1506-17.

346. Blasius, A.L.; Giurisato, E.; Cella, M.; Schreiber, R.D.; Shaw, A.S.; Colonna, M. Bone marrow stromal cell antigen 2 is a specific marker of type I IFN-producing cells in the naive mouse, but a promiscuous cell surface antigen following IFN stimulation. J. Immunol. 2006, 177, 3260-5.

347. Erikson, E.; Adam, T.; Schmidt, S.; Lehmann-Koch, J.; Over, B.; Goffinet, C.; Harter, C.; Bekeredjian-Ding, I.; Sertel, S.; Lasitschka, F.; et al. In vivo expression profile of the antiviral restriction factor and tumor-targeting antigen CD317/BST-2/HM1.24/tetherin in humans. Proc. Natl. Acad. Sci. U. S. A. 2011, 108, 13688-93.

348. Goto, T.; Kennel, S.J.; Abe, M.; Takishita, M.; Kosaka, M.; Solomon, A.; Saito, $S$. A novel membrane antigen selectively expressed on terminally differentiated human B cells. Blood 1994, 84, 1922-30.

349. Ishikawa, J.; Kaisho, T.; Tomizawa, H.; Lee, B.O.; Kobune, Y.; Inazawa, J.; Oritani, K.; Itoh, M.; Ochi, T.; Ishihara, K. Molecular cloning and chromosomal mapping of a bone marrow stromal cell surface gene, BST2, that may be involved in pre-B-cell growth. Genomics 1995, 26, 527-34. 
350. Kupzig, S.; Korolchuk, V.; Rollason, R.; Sugden, A.; Wilde, A.; Banting, G. Bst$2 / \mathrm{HM} 1.24$ is a raft-associated apical membrane protein with an unusual topology. Traffic 2003, 4, 694-709.

351. Andrew, A.J.; Miyagi, E.; Kao, S.; Strebel, K. The formation of cysteine-linked dimers of BST-2/tetherin is important for inhibition of HIV-1 virus release but not for sensitivity to Vpu. Retrovirology 2009, 6, 80.

352. Swiecki, M.; Scheaffer, S.M.; Allaire, M.; Fremont, D.H.; Colonna, M.; Brett, T.J. Structural and biophysical analysis of BST-2/tetherin ectodomains reveals an evolutionary conserved design to inhibit virus release. J. Biol. Chem. 2011, 286, 2987-97.

353. Welbourn, S.; Kao, S.; Du Pont, K.E.; Andrew, A.J.; Berndsen, C.E.; Strebel, K. Positioning of cysteine residues within the $\mathrm{N}$-terminal portion of the BST2/tetherin ectodomain is important for functional dimerization of BST-2. J. Biol. Chem. 2015, 290, 3740-3751.

354. Hammonds, J.; Wang, J.-J.; Yi, H.; Spearman, P. Immunoelectron microscopic evidence for Tetherin/BST2 as the physical bridge between HIV-1 virions and the plasma membrane. PLoS Pathog. 2010, 6, e1000749.

355. Rollason, R.; Korolchuk, V.; Hamilton, C.; Schu, P.; Banting, G. Clathrinmediated endocytosis of a lipid-raft-associated protein is mediated through a dual tyrosine motif. J. Cell Sci. 2007, 120, 3850-8.

356. Masuyama, N.; Kuronita, T.; Tanaka, R.; Muto, T.; Hirota, Y.; Takigawa, A.; Fujita, H.; Aso, Y.; Amano, J.; Tanaka, Y. HM1.24 is internalized from lipid rafts by clathrin-mediated endocytosis through interaction with alpha-adaptin. J. Biol. Chem. 2009, 284, 15927-41.

357. Matsuda, A.; Suzuki, Y.; Honda, G.; Muramatsu, S.; Matsuzaki, O.; Nagano, Y.; Doi, T.; Shimotohno, K.; Harada, T.; Nishida, E.; et al. Large-scale identification and characterization of human genes that activate NF-kappaB and MAPK signaling pathways. Oncogene 2003, 22, 3307-18.

358. Galão, R.P.; Le Tortorec, A.; Pickering, S.; Kueck, T.; Neil, S.J.D. Innate sensing of HIV-1 assembly by Tetherin induces NFKB-dependent proinflammatory responses. Cell Host Microbe 2012, 12, 633-44.

359. Neil, S.J.D.; Zang, T.; Bieniasz, P.D. Tetherin inhibits retrovirus release and is antagonized by HIV-1 Vpu. Nature 2008, 451, 425-30.

360. Perez-Caballero, D.; Zang, T.; Ebrahimi, A.; McNatt, M.W.; Gregory, D.A.; Johnson, M.C.; Bieniasz, P.D. Tetherin inhibits HIV-1 release by directly tethering virions to cells. Cell 2009, 139, 499-511.

361. Neil, S.J.D. The antiviral activities of tetherin. In Intrinsic Immunity, Current Topics in Microbiology and Immunology; Cullen, B.R., Ed.; Springer-Verlag Berlin Heidelberg, 2013; pp. 67-104.

362. Sarojini, S.; Theofanis, T.; Reiss, C.S. Interferon-induced tetherin restricts vesicular stomatitis virus release in neurons. DNA Cell Biol. 2011, 30, 965-74.

363. Jones, P.H.; Maric, M.; Madison, M.N.; Maury, W.; Roller, R.J.; Okeoma, C.M. BST-2/tetherin-mediated restriction of chikungunya (CHIKV) VLP budding is counteracted by CHIKV non-structural protein 1 (nsP1). Virology 2013, 438, 3749.

364. Ooi, Y.S.; Dubé, M.; Kielian, M. BST2/tetherin inhibition of alphavirus exit. Viruses 2015, 7, 2147-67.

365. Radoshitzky, S.R.; Dong, L.; Chi, X.; Clester, J.C.; Retterer, C.; Spurgers, K.; Kuhn, J.H.; Sandwick, S.; Ruthel, G.; Kota, K.; et al. Infectious Lassa virus, but not filoviruses, is restricted by BST-2/tetherin. J. Virol. 2010, 84, 10569-80. 
366. Yondola, M.A.; Fernandes, F.; Belicha-Villanueva, A.; Uccelini, M.; Gao, Q.; Carter, C.; Palese, P. Budding capability of the influenza virus neuraminidase can be modulated by tetherin. J. Virol. 2011, 85, 2480-91.

367. Douglas, J.L.; Gustin, J.K.; Viswanathan, K.; Mansouri, M.; Moses, A. V.; Früh, K. The great escape: viral strategies to counter BST-2/tetherin. PLoS Pathog. 2010, 6, e1000913.

368. Miyakawa, K.; Matsunaga, S.; Watashi, K.; Sugiyama, M.; Kimura, H.; Yamamoto, N.; Mizokami, M.; Wakita, T.; Ryo, A. Molecular dissection of HBV evasion from restriction factor tetherin: A new perspective for antiviral cell therapy. Oncotarget 2015, 6, 21840-52.

369. Pan, X.-B.; Han, J.-C.; Cong, X.; Wei, L. BST2/tetherin inhibits dengue virus release from human hepatoma cells. PLoS One 2012, 7, e51033.

370. Pan, X.-B.; Qu, X.-W.; Jiang, D.; Zhao, X.-L.; Han, J.-C.; Wei, L. BST2/Tetherin inhibits hepatitis $\mathrm{C}$ virus production in human hepatoma cells. Antiviral Res. 2013, 98, 54-60.

371. Amet, T.; Byrd, D.; Hu, N.; Sun, Q.; Li, F.; Zhao, Y.; Hu, S.; Grantham, A.; Yu, Q. BST-2 expression in human hepatocytes is inducible by all three types of interferons and restricts production of hepatitis C virus. Curr. Mol. Med. 2014, 14, 349-60.

372. Lopez, L.A.; Yang, S.J.; Hauser, H.; Exline, C.M.; Haworth, K.G.; Oldenburg, J.; Cannon, P.M. Ebola virus glycoprotein counteracts BST-2/Tetherin restriction in a sequence-independent manner that does not require tetherin surface removal. J. Virol. 2010, 84, 7243-55.

373. Kühl, A.; Banning, C.; Marzi, A.; Votteler, J.; Steffen, I.; Bertram, S.; Glowacka, I.; Konrad, A.; Stürzl, M.; Guo, J.-T.; et al. The Ebola virus glycoprotein and HIV$1 \mathrm{Vpu}$ employ different strategies to counteract the antiviral factor tetherin. J. Infect. Dis. 2011, 204 Suppl, S850-60.

374. Santa-Marta, M.; de Brito, P.M.; Godinho-Santos, A.; Goncalves, J. Host Factors and HIV-1 Replication: Clinical Evidence and Potential Therapeutic Approaches. Front. Immunol. 2013, 4, 343.

375. Fitzpatrick, K.; Skasko, M.; Deerinck, T.J.; Crum, J.; Ellisman, M.H.; Guatelli, J. Direct restriction of virus release and incorporation of the interferon-induced protein BST-2 into HIV-1 particles. PLoS Pathog. 2010, 6, e1000701.

376. Strauss, J.D.; Hammonds, J.E.; Yi, H.; Ding, L.; Spearman, P.; Wright, E.R. Three-Dimensional Structural Characterization of HIV-1 Tethered to Human Cells. J. Virol. 2016, 90, 1507-21.

377. Dubé, M.; Bego, M.G.; Paquay, C.; Cohen, É.A. Modulation of HIV-1-host interaction: role of the Vpu accessory protein. Retrovirology 2010, 7, 114.

378. Venkatesh, S.; Bieniasz, P.D. Mechanism of HIV-1 virion entrapment by tetherin. PLoS Pathog. 2013, 9, e1003483.

379. Mahauad-Fernandez, W.D.; Jones, P.H.; Okeoma, C.M. Critical role for bone marrow stromal antigen 2 in acute Chikungunya virus infection. J. Gen. Virol. 2014, 95, 2450-61.

380. Liberatore, R.A.; Bieniasz, P.D. Tetherin is a key effector of the antiretroviral activity of type I interferon in vitro and in vivo. Proc. Natl. Acad. Sci. U. S. A. 2011, 108, 18097-101.

381. Jones, P.H.; Mehta, H. V.; Maric, M.; Roller, R.J.; Okeoma, C.M. Bone marrow stromal cell antigen 2 (BST-2) restricts mouse mammary tumor virus (MMTV) replication in vivo. Retrovirology 2012, 9, 10.

382. Li, S.X.; Barrett, B.S.; Heilman, K.J.; Messer, R.J.; Liberatore, R.A.; Bieniasz, 
P.D.; Kassiotis, G.; Hasenkrug, K.J.; Santiago, M.L. Tetherin promotes the innate and adaptive cell-mediated immune response against retrovirus infection in vivo. J. Immunol. 2014, 193, 306-16.

383. Mahauad-Fernandez, W.D.; Okeoma, C.M. The role of BST-2/Tetherin in host protection and disease manifestation. Immunity, Inflamm. Dis. 2016, 4, 4-23.

384. Van Damme, N.; Goff, D.; Katsura, C.; Jorgenson, R.L.; Mitchell, R.; Johnson, M.C.; Stephens, E.B.; Guatelli, J. The interferon-induced protein BST-2 restricts HIV-1 release and is downregulated from the cell surface by the viral Vpu protein. Cell Host Microbe 2008, 3, 245-52.

385. Abada, P.; Noble, B.; Cannon, P.M. Functional domains within the human immunodeficiency virus type 2 envelope protein required to enhance virus production. J. Virol. 2005, 79, 3627-38.

386. Le Tortorec, A.; Neil, S.J.D. Antagonism to and intracellular sequestration of human tetherin by the human immunodeficiency virus type 2 envelope glycoprotein. J. Virol. 2009, 83, 11966-78.

387. Sauter, D.; Schindler, M.; Specht, A.; Landford, W.N.; Münch, J.; Kim, K.-A.; Votteler, J.; Schubert, U.; Bibollet-Ruche, F.; Keele, B.F.; et al. Tetherin-driven adaptation of $\mathrm{Vpu}$ and Nef function and the evolution of pandemic and nonpandemic HIV-1 strains. Cell Host Microbe 2009, 6, 409-21.

388. Jia, B.; Serra-Moreno, R.; Neidermyer, W.; Rahmberg, A.; Mackey, J.; Fofana, I. Ben; Johnson, W.E.; Westmoreland, S.; Evans, D.T. Species-specific activity of SIV Nef and HIV-1 Vpu in overcoming restriction by tetherin/BST2. PLoS Pathog. 2009, 5, e1000429.

389. Serra-Moreno, R.; Evans, D.T. Adaptation of human and simian immunodeficiency viruses for resistance to tetherin/BST-2. Curr. HIV Res. 2012, 10, 277-82.

390. Mansouri, M.; Viswanathan, K.; Douglas, J.L.; Hines, J.; Gustin, J.; Moses, A. V.; Früh, K. Molecular mechanism of BST2/tetherin downregulation by K5/MIR2 of Kaposi's sarcoma-associated herpesvirus. J. Virol. 2009, 83, 9672-81.

391. Blondeau, C.; Pelchen-Matthews, A.; Mlcochova, P.; Marsh, M.; Milne, R.S.B.; Towers, G.J. Tetherin restricts herpes simplex virus 1 and is antagonized by glycoprotein M. J. Virol. 2013, 87, 13124-33.

392. Taylor, J.K.; Coleman, C.M.; Postel, S.; Sisk, J.M.; Bernbaum, J.G.; Venkataraman, T.; Sundberg, E.J.; Frieman, M.B. Severe Acute Respiratory Syndrome Coronavirus ORF7a Inhibits Bone Marrow Stromal Antigen 2 Virion Tethering through a Novel Mechanism of Glycosylation Interference. J. Virol. 2015, 89, 11820-33.

393. Ohta, K.; Goto, H.; Yumine, N.; Nishio, M. Human parainfluenza virus type 2 V protein inhibits and antagonizes tetherin. J. Gen. Virol. 2016, 97, 561-70.

394. Bampi, C.; Rasga, L.; Roux, L. Antagonism to human BST-2/tetherin by Sendai virus glycoproteins. J. Gen. Virol. 2013, 94, 1211-9.

395. Kaletsky, R.L.; Francica, J.R.; Agrawal-Gamse, C.; Bates, P. Tetherin-mediated restriction of filovirus budding is antagonized by the Ebola glycoprotein. Proc. Natl. Acad. Sci. U. S. A. 2009, 106, 2886-91.

396. Vigan, R.; Neil, S.J.D. Determinants of tetherin antagonism in the transmembrane domain of the human immunodeficiency virus type $1 \mathrm{Vpu}$ protein. J. Virol. 2010, 84, 12958-70.

397. Dubé, M.; Roy, B.B.; Guiot-Guillain, P.; Binette, J.; Mercier, J.; Chiasson, A.; Cohen, E.A. Antagonism of tetherin restriction of HIV-1 release by Vpu involves binding and sequestration of the restriction factor in a perinuclear compartment. 
PLoS Pathog. 2010, 6, e1000856.

398. Skasko, M.; Wang, Y.; Tian, Y.; Tokarev, A.; Munguia, J.; Ruiz, A.; Stephens, E.B.; Opella, S.J.; Guatelli, J. HIV-1 Vpu protein antagonizes innate restriction factor BST-2 via lipid-embedded helix-helix interactions. J. Biol. Chem. 2012, 287, 58-67.

399. Miyagi, E.; Andrew, A.J.; Kao, S.; Strebel, K. Vpu enhances HIV-1 virus release in the absence of Bst-2 cell surface down-modulation and intracellular depletion. Proc. Natl. Acad. Sci. 2009, 106, 2868-2873.

400. Goffinet, C.; Allespach, I.; Homann, S.; Tervo, H.-M.; Habermann, A.; Rupp, D.; Oberbremer, L.; Kern, C.; Tibroni, N.; Welsch, S.; et al. HIV-1 antagonism of CD317 is species specific and involves Vpu-mediated proteasomal degradation of the restriction factor. Cell Host Microbe 2009, 5, 285-97.

401. Rollason, R.; Dunstan, K.; Billcliff, P.G.; Bishop, P.; Gleeson, P.; Wise, H.; Digard, P.; Banting, G. Expression of HIV-1 Vpu leads to loss of the viral restriction factor CD317/Tetherin from lipid rafts and its enhanced lysosomal degradation. PLoS One 2013, 8, e75680.

402. Janvier, K.; Roy, N.; Berlioz-Torrent, C. Role of the endosomal ESCRT machinery in HIV-1 Vpu-induced down-regulation of BST2/tetherin. Curr. HIV Res. 2012, 10, 315-20.

403. Douglas, J.L.; Viswanathan, K.; McCarroll, M.N.; Gustin, J.K.; Früh, K.; Moses, A. V. Vpu directs the degradation of the human immunodeficiency virus restriction factor BST-2/Tetherin via a beta\}TrCP-dependent mechanism. J. Virol. 2009, 83, 7931-47.

404. Mangeat, B.; Gers-Huber, G.; Lehmann, M.; Zufferey, M.; Luban, J.; Piguet, V. HIV-1 Vpu neutralizes the antiviral factor Tetherin/BST-2 by binding it and directing its beta-TrCP2-dependent degradation. PLoS Pathog. 2009, 5, e1000574.

405. Schmidt, S.; Fritz, J. V.; Bitzegeio, J.; Fackler, O.T.; Keppler, O.T. HIV-1 vpu blocks recycling and biosynthetic transport of the intrinsic immunity factor CD317/tetherin to overcome the virion release restriction. MBio 2011.

406. Lewinski, M.K.; Jafari, M.; Zhang, H.; Opella, S.J.; Guatelli, J. Membrane Anchoring by a C-terminal Tryptophan Enables HIV-1 Vpu to Displace Bone Marrow Stromal Antigen 2 (BST2) from Sites of Viral Assembly. J. Biol. Chem. 2015, 290, 10919-33.

407. Kühl, A.; Hoffmann, M.; Müller, M.A.; Munster, V.J.; Gnirss, K.; Kiene, M.; Tsegaye, T.S.; Behrens, G.; Herrler, G.; Feldmann, H.; et al. Comparative analysis of Ebola virus glycoprotein interactions with human and bat cells. J. Infect. Dis. 2011, 204 Suppl, S840-9.

408. Hoffmann, M.; Nehlmeier, I.; Brinkmann, C.; Krähling, V.; Behner, L.; Moldenhauer, A.-S.; Krüger, N.; Nehls, J.; Schindler, M.; Hoenen, T.; et al. Tetherin inhibits Nipah virus but not Ebola virus replication in fruit bat cells. J. Virol. 2018.

409. Panchal, R.G.; Ruthel, G.; Kenny, T.A.; Kallstrom, G.H.; Lane, D.; Badie, S.S.; Li, L.; Bavari, S.; Aman, M.J. In vivo oligomerization and raft localization of Ebola virus protein VP40 during vesicular budding. Proc. Natl. Acad. Sci. 2003.

410. Gustin, J.K.; Bai, Y.; Moses, A. V.; Douglas, J.L. Ebola Virus Glycoprotein Promotes Enhanced Viral Egress by Preventing Ebola VP40 From Associating With the Host Restriction Factor BST2/Tetherin. J. Infect. Dis. 2015, 212 Suppl, S181-90.

411. Feagins, A.R.; Basler, C.F. The VP40 Protein of Marburg Virus Exhibits Impaired 
Budding and Increased Sensitivity to Human Tetherin following Mouse Adaptation. J. Virol. 2014.

412. Morrison, J.H.; Guevara, R.B.; Marcano, A.C.; Saenz, D.T.; Fadel, H.J.; Rogstad, D.K.; Poeschla, E.M. Feline Immunodeficiency Virus Envelope Glycoproteins Antagonize Tetherin through a Distinctive Mechanism That Requires Virion Incorporation. J. Virol. 2014.

413. Gnirß, K.; Fiedler, M.; Krämer-Kühl, A.; Bolduan, S.; Mittler, E.; Becker, S.; Schindler, M.; Pöhlmann, S. Analysis of determinants in filovirus glycoproteins required for tetherin antagonism. Viruses 2014, 6, 1654-71.

414. Vande Burgt, N.H.; Kaletsky, R.L.; Bates, P. Requirements within the Ebola viral glycoprotein for tetherin antagonism. Viruses 2015, 7, 5587-5602.

415. Brinkmann, C.; Nehlmeier, I.; Walendy-Gnirß, K.; Nehls, J.; González Hernández, M.; Hoffmann, M.; Qiu, X.; Takada, A.; Schindler, M.; Pöhlmann, S. The Tetherin Antagonism of the Ebola Virus Glycoprotein Requires an Intact Receptor-Binding Domain and Can Be Blocked by GP1-Specific Antibodies. J. Virol. 2016, 90, 11075-11086.

416. Hacke, M.; Björkholm, P.; Hellwig, A.; Himmels, P.; Ruiz de Almodóvar, C.; Brügger, B.; Wieland, F.; Ernst, A.M. Inhibition of Ebola virus glycoproteinmediated cytotoxicity by targeting its transmembrane domain and cholesterol. Nat. Commun. 2015, 6, 7688.

417. Watt, A.; Moukambi, F.; Banadyga, L.; Groseth, A.; Callison, J.; Herwig, A.; Ebihara, H.; Feldmann, H.; Hoenen, T. A Novel Life Cycle Modeling System for Ebola Virus Shows a Genome Length-Dependent Role of VP24 in Virus Infectivity. J. Virol. 2014, 88, 10511-10524.

418. Wang, Z.; Li, J.; Fu, Y.; Zhao, Z.; Zhang, C.; Li, N.; Li, J.; Cheng, H.; Jin, X.; Lu, B.; et al. A Rapid Screen for Host-Encoded miRNAs with Inhibitory Effects against Ebola Virus Using a Transcription- and Replication-Competent VirusLike Particle System. Int. J. Mol. Sci. 2018, 19.

419. Zmora, P.; Moldenhauer, A.-S.; Hofmann-Winkler, H.; Pöhlmann, S. TMPRSS2 Isoform 1 Activates Respiratory Viruses and Is Expressed in Viral Target Cells. PLoS One 2015, 10, e0138380.

420. Wool-Lewis, R.J.; Bates, P. Endoproteolytic processing of the ebola virus envelope glycoprotein: cleavage is not required for function. J. Virol. 1999, 73, 1419-26.

421. Neumann, G.; Feldmann, H.; Watanabe, S.; Lukashevich, I. Reverse Genetics Demonstrates that Proteolytic Processing of the Ebola Virus Glycoprotein Is Not Essential for Replication in Cell Culture. J. Virol. 2002, 76, 406-410.

422. Neumann, G.; Geisbert, T.W.; Ebihara, H.; Geisbert, J.B.; Daddario-DiCaprio, K.M.; Feldmann, H.; Kawaoka, Y. Proteolytic Processing of the Ebola Virus Glycoprotein Is Not Critical for Ebola Virus Replication in Nonhuman Primates. J. Virol. 2007, 81, 2995-2998.

423. Hook, G.; Jacobsen, J.S.; Grabstein, K.; Kindy, M.; Hook, V. Cathepsin B is a New Drug Target for Traumatic Brain Injury Therapeutics: Evidence for E64d as a Promising Lead Drug Candidate. Front. Neurol. 2015, 6, 178.

424. Sudhan, D.R.; Siemann, D.W. Cathepsin L targeting in cancer treatment. Pharmacol. Ther. 2015, 155, 105-16.

425. Wang, S.-Q.; Du, Q.-S.; Zhao, K.; Li, A.-X.; Wei, D.-Q.; Chou, K.-C. Virtual screening for finding natural inhibitor against cathepsin- $L$ for SARS therapy. Amino Acids 2007, 33, 129-35.

426. Brana, C.; Benham, C.D.; Sundstrom, L.E. Calpain activation and inhibition in 
organotypic rat hippocampal slice cultures deprived of oxygen and glucose. Eur. J. Neurosci. 1999, 11, 2375-84.

427. Gewies, A.; Grimm, S. Cathepsin-B and cathepsin-L expression levels do not correlate with sensitivity of tumour cells to TNF-alpha-mediated apoptosis. Br. J. Cancer 2003, 89, 1574-80.

428. Simmons, G.; Gosalia, D.N.; Rennekamp, A.J.; Reeves, J.D.; Diamond, S.L.; Bates, $P$. Inhibitors of cathepsin $L$ prevent severe acute respiratory syndrome coronavirus entry. Proc. Natl. Acad. Sci. U. S. A. 2005, 102, 11876-81.

429. Siklos, M.; BenAissa, M.; Thatcher, G.R.J. Cysteine proteases as therapeutic targets: does selectivity matter? A systematic review of calpain and cathepsin inhibitors. Acta Pharm. Sin. B 2015, 5, 506-19.

430. Wool-Lewis, R.J.; Bates, P. Characterization of Ebola virus entry by using pseudotyped viruses: identification of receptor-deficient cell lines. J. Virol. 1998, 72, 3155-3160.

431. Yang, Z.; Delgado, R.; Xu, L.; Todd, R.F.; Nabel, E.G.; Sanchez, A.; Nabel, G.J. Distinct cellular interactions of secreted and transmembrane Ebola virus glycoproteins. Science 1998, 279, 1034-7.

432. Dube, D.; Schornberg, K.L.; Shoemaker, C.J.; Delos, S.E.; Stantchev, T.S.; Clouse, K.A.; Broder, C.C.; White, J.M. Cell adhesion-dependent membrane trafficking of a binding partner for the ebolavirus glycoprotein is a determinant of viral entry. Proc. Natl. Acad. Sci. U. S. A. 2010, 107, 16637-42.

433. Park, J.-E.; Li, K.; Barlan, A.; Fehr, A.R.; Perlman, S.; McCray, P.B.; Gallagher, T. Proteolytic processing of Middle East respiratory syndrome coronavirus spikes expands virus tropism. Proc. Natl. Acad. Sci. 2016, 113, 12262-12267.

434. Licata, J.M.; Johnson, R.F.; Han, Z.; Harty, R.N. Contribution of ebola virus glycoprotein, nucleoprotein, and VP24 to budding of VP40 virus-like particles. J. Virol. 2004, 78, 7344-51.

435. Lopez, L.A.; Yang, S.J.; Exline, C.M.; Rengarajan, S.; Haworth, K.G.; Cannon, P.M. Anti-Tetherin Activities of HIV-1 Vpu and Ebola Virus Glycoprotein Do Not Involve Removal of Tetherin from Lipid Rafts. J. Virol. 2012, 86, 5467-5480.

436. Teese, M.G.; Langosch, D. Role of GxxxG Motifs in Transmembrane Domain Interactions. Biochemistry 2015, 54, 5125-35.

437. Takada, A.; Feldmann, H.; Stroeher, U.; Bray, M.; Watanabe, S.; Ito, H.; McGregor, M.; Kawaoka, Y. Identification of protective epitopes on ebola virus glycoprotein at the single amino acid level by using recombinant vesicular stomatitis viruses. J. Virol. 2003, 77, 1069-74.

438. Garbutt, M.; Liebscher, R.; Wahl-Jensen, V.; Jones, S.; Moller, P.; Wagner, R.; Volchkov, V.; Klenk, H.-D.; Feldmann, H.; Stroher, U. Properties of ReplicationCompetent Vesicular Stomatitis Virus Vectors Expressing Glycoproteins of Filoviruses and Arenaviruses. J. Virol. 2004, 78, 5458-5465.

439. Brinkmann, C.; Hoffmann, M.; Lübke, A.; Nehlmeier, I.; Krämer-Kühl, A.; Winkler, M.; Pöhlmann, S. The glycoprotein of vesicular stomatitis virus promotes release of virus-like particles from tetherin-positive cells. PLoS One 2017, 12, e0189073. 


\section{Appendix}

\subsection{List of abbreviations}

\begin{tabular}{ll} 
ADAM17 & A disintegrin and metalloproteinase 17 \\
ASGPR & Asialoglycoprotein receptors \\
ASGPR-1 & Asialoglycoprotein receptor-1 \\
BDBV & Bundibugyo virus \\
BOMV & Bombali virus \\
BSL4 & Biosecurity level 4 \\
BST-2 & Bone marrow stromal antigen 2 \\
CARD & caspase recruitment domains \\
CatB & Cathepsin B \\
CatL & Cathepsin L \\
CD209 & Cluster of differentiation 209 \\
CD317 & Cluster of differentiation 317 \\
CD4+ & Cluster of differentiation 4 \\
Cdc42 & Cell division control protein 42 homolog \\
CHIKV & Chikungunya virus \\
CLR & C-type lectin receptors \\
cRNA & Complementary ribonucleic acid \\
CTL & C-type lectins \\
Cys & Cysteine \\
DC & Dendritic cells \\
DC-SIGN & Dendritic cell-specific intercellular adhesion molecule-3-grabbing \\
& non-integrin \\
DENV & Dengue virus \\
DNA & Deoxyribonucleic acid \\
DRC & Democratic Republic of Congo \\
dsRNA & Double-stranded ribonuclec acid \\
EBOV & Ebola virus \\
EBOV-GP & Ebola virus glycoprotein \\
EGFR & Epidermal growth factor receptor \\
ER & Endoplasmic reticulum \\
EVD & Ebola virus disease \\
Gas6 & Growth arrest-specific 6 \\
GDP & Guanidine diphosphate \\
GED & GTPase effector domain \\
GP & Glycoprotein \\
GP0 & Glycoprotein 0 \\
GP1 & Glycoprotein 1 \\
GP2 & Glycoprotein 2 \\
GPI & Glycosylphosphatidylinositol \\
GTP & Guanidine triphosphate \\
GTPase & Guanosine triphosphatase \\
HCV & Hepatitis C virus \\
HIV-1 & Human immunodeficiency virus 1 \\
\hline
\end{tabular}




\begin{tabular}{ll} 
HIV-2 & Human immunodeficiency virus 2 \\
hPIV-2 & Human parainfluenza virus type 2 \\
HR & Heptad repeat \\
HSV & Herpes simplex virus \\
IAV & Influenza A virus \\
ICAM-1 & Intercellular cell adhesion molecule-1 \\
IFITM & Interferon-induced transmembrane protein \\
IFL & Internal fusion loop \\
IFN & Interferon \\
IFNR & Interferon receptor \\
IFN-y & Interferon gamma \\
Ig & Immunoglobulin \\
IgM & Immunoglobulin M \\
IgV & Immunoglobulin variable \\
IRF & Interferon-regulatory factors \\
ISG & Interferon stimulated gene \\
JAK & Janus-kinases \\
KPNA & Karyopherin a \\
KSHV & Kaposi's sarcoma-associated herpesvirus \\
LGP2 & Laboratory of Genetics and Physiology 2 \\
LLOV & Lloviu virus \\
LPS & Lipopolysaccharide \\
LRR & Leucine-rich repeats \\
LSECtin & Liver and lymph node sinusoidal endothelial cell C-type lectin \\
L-SIGN & Lymph node-specific intercellular adhesion molecule-3-grabbing \\
MARV & integrin \\
MAVS & Marburg virus \\
MD & Mitochondrial antiviral signaling protein \\
MD5A & Middle domain \\
MERS & Melanoma differentiation-associated protein 5 \\
MFG-E8 & Middle east respiratory syndrome \\
MGL & Milk fat globule-epidermal growth factor-factor 8 \\
MLD & Macrophage galactose C-type lectin \\
MLV & Mucin-like domain \\
mRNA & Murine leukemia viruses \\
Mx & Messenger ribonucleic acid \\
MyD88 & Myxovirus resistance protein \\
Nedd4 & Myeloid differentiation factor 88 \\
NF-kB & Neural precursor cell expressed developmentally down-regulated \\
NHP & protein 4 \\
NK & Nuclear factor kappa-light-chain-enhancer of activated B cells \\
NLR & Non-human primate \\
NOD & NOD-like receptors \\
NP & Nucleotide-binding oligomerization domain \\
& Nucleoprotein \\
\hline
\end{tabular}




$\begin{array}{ll}\text { NPC1 } & \text { Niemann-Pick C1 } \\ \text { NPC2 } & \text { Niemann-Pick C2 } \\ \text { NTD } & \text { N-terminal domain } \\ \text { OAS } & \text { 2'-5'-oligo-adenylate synthetase } \\ \text { OASL } & \text { OAS-like } \\ \text { ORF } & \text { Open reading frame } \\ \text { PACT } & \text { PKR activator } \\ \text { Pak1 } & \text { Serine/threonine-protein kinase } \\ \text { PAMP } & \text { Pathogen-associated molecular patterns } \\ \text { PECAM-1 } & \text { Platelet/endothelial cell adhesion molecule-1 } \\ \text { PHEIC } & \text { Public Health Emergency of International Concern } \\ \text { PI3K } & \text { Phosphatidylinositol 3-kinases } \\ \text { PKC } & \text { Protein kinase C } \\ \text { PKR } & \text { Protein kinase R } \\ \text { PRR } & \text { Pattern recognition receptors } \\ \text { PtdSer } & \text { Phosphatidylserine } \\ \text { PTK } & \text { Protein tyrosine kinase } \\ \text { PVEERs } & \text { Phosphatidylserine-mediated virus entry enhancing receptors } \\ \text { Rac1 } & \text { Ras-related C3 botulinum toxin substrate 1 } \\ \text { RAVV } & \text { Ravn virus } \\ \text { RBD } & \text { Receptor binding domain } \\ \text { RESTV } & \text { Reston virus } \\ \text { RIG-I } & \text { Retinoic acid-inducible gene-I } \\ \text { RLR } & \text { RIG-I-like receptor } \\ \text { RNA } & \text { Ribonucleic acid } \\ \text { RNase L } & \text { Ribonuclease L } \\ \text { RNP } & \text { Ribonucleoprotein } \\ \text { Rsp5 } & \text { Reversion of Spt phenotype } \\ \text { RT-PCR } & \text { Reverse transcription polymerase chain reaction } \\ \text { SARS-CoV } & \text { Severe acute respiratory syndrome coronavirus } \\ \text { SGP } & \text { Soluble Glycoprotein } \\ \text { siRNA } & \text { Small interfering ribonucleic acid } \\ \text { SIV } & \text { Simian immunodeficiency virus } \\ \text { SP } & \text { Signal peptide } \\ \text { SSD } & \text { Sterol sensing domain } \\ \text { SSGP } & \text { Small soluble Glycoprotein } \\ \text { SSRNA } & \text { Single-stranded ribonucleic acid } \\ \text { STAT } & \text { Signal transducer and activator of transcription } \\ \text { SUDV } & \text { Sudan virus } \\ \text { TACE } & \text { Tumor necrosis factor alpha-converting enzyme } \\ \text { TAFV } & \text { Taï Forest virus } \\ \text { TAM } & \text { Tyro-3, Axl, and Mer receptors } \\ \text { TBK1 } & \text { TANK-binding kinase 1 } \\ \text { TGN } & \text { Trans-Golgi network } \\ \text { TIM } & \text { T-cell immunoglobulin and mucin-domain containing protein } \\ \text { TIR } & \text { Toll-IL-1 receptor } \\ & \end{array}$




$\begin{array}{ll}\text { TLR } & \text { Toll-like receptor } \\ \text { TMD } & \text { Transmembrane domain } \\ \text { TRAF } & \text { TNF receptor-associated factor } \\ \text { TRIF } & \text { TIR-domain-containing adaptor-inducing IFN- factor } \\ \text { TRIM } & \text { Tripartite motif protein } \\ \text { trVLP } & \text { Transcription- and replication-competent virus-like particle } \\ \text { Tsg101 } & \text { Tumor susceptibility gene 101 } \\ \text { TYK2 } & \text { Tyrosine kinase } 2 \\ \text { VCAM-1 } & \text { Vascular cell adhesion molecule-1 } \\ \text { VLP } & \text { Virus-like particle } \\ \text { VP24 } & \text { Viral protein with molecular mass of } 24 \text { kilodalton } \\ \text { VP30 } & \text { Viral protein with molecular mass of } 30 \text { kilodalton } \\ \text { VP35 } & \text { Viral protein with molecular mass of } 35 \text { kilodalton } \\ \text { VP40 } & \text { Viral protein with molecular mass of } 40 \text { kilodalton } \\ \text { Vpu } & \text { Viral protein U } \\ \text { VSV } & \text { Vesicular stomatitis virus } \\ \text { VSV-EBOV-GP } & \text { Vesicular stomatitis virus with Ebola virus glycoprotein } \\ \text { WHO } & \text { World Health Organization }\end{array}$




\subsection{Acknowledgements}

First of all, I would like to thank Prof. Dr. Stefan Pöhlmann for giving me the opportunity to work on this project. I appreciate the valuable suggestions and your help during the whole course of my thesis.

I would like to thank the members of my thesis committee, Prof. Dr. Lutz Walter and Prof. Dr. Stephan Becker for their support and their ideas.

I would also like to thank Dr. Markus Hoffmann for his supervision, his constant support, advice and interesting discussions during my thesis.

I would like to thank the National Council on Science and Technology of Mexico (CONACYT), the DAAD and the MayaNet Project from the Erasmus Mundus partnership for financial support.

Thanks to all the members (current and former) of the Infection Biology Unit. You all made going to work fun. I really appreciate the kind and friendly atmosphere. Anna, Inga and Sabine thanks for all your help in the lab, I learned a lot from you. Kathrin thank you for all the help with the paper work before and during my stay in Germany. Also, thanks to Heike for the advice and valuable comments on my thesis. Hannah, Lisa and Astrid, you made the time in the lab less boring and it was nice to have someone to joke around with. Thank you to the participants of the coffee breaks, Mario Kart parties and free German lessons, I had a lot of great moments.

A special thanks goes to Constantin for his support and patience particularly during the final phase of my thesis. But most importantly for making me laugh specially when times were tough.

Thanks to my friends in Mexico Erick, Karen, Lucero for all the cheering and being there for me even if we could only see each other once a year. Also, to Vero, Omar, Mario and Dr. Leopoldo for their support and help.

Finally, and most important I would like to thank my parents and my brother for all the support they have given me over the years, their love and cheering. Sin su apoyo no hubiera estado aquí. ¡Gracias, los quiero! 


\subsection{Curriculum Vitae}

Mariana González Hernández, M.Sc.

Born: 04. October 1988 in Mexico City, Mexico

E-Mail: mariana.gonzalezh@hotmail.com

\section{Academic Education}

08/2015-03/2019 PhD within the Graduate School "Emerging Infectious

Diseases" (EIDIS)

Georg-August University School of Science (GAUSS)

Göttingen, Germany

Thesis "Interactions of the Ebola virus glycoprotein with host cell factors during viral entry and release"

Supervisor: Prof. Dr. Stefan Pöhlmann

08/2012 - 08/2014 M.Sc., Master in Health Science

Escuela Superior de Medicina - Instituto Politécnico Nacional Mexico City, Mexico

Thesis "Identification and validation of epitopes of Dengue virus serotypes for serological diagnosis"

Supervisors: Dr. Leopoldo Aguilar Faisal

Dr. Adolfo Chávez Negrete

Graduated with Honors

08/2007 - 12/2011 Bachelor in Biochemistry Engineering

Escuela Nacional de Ciencias Biológicas- Instituto Politécnico Nacional

Mexico City, Mexico

Thesis "Activation and retrieval of the four serotypes of Dengue virus and its identification using RT-PCR"

Supervisors: M.Sc. Verónica Alcántara Farfán

M.Sc. Arturo Barbachano Guerrero

08/2004 - 07/2007 High School, Chemical Technician

Centro de Estudios Científicos y Tecnológicos No.6 Miguel

Othón de Mendizábal-Instituto Politécnico Nacional

Mexico City, Mexico 


\section{Honors and Awards}

10/2015 - present Scholarship holder within the DAAD country-related cooperation program with the National Council on Science and Technology of Mexico (CONACYT)

08/2015 - 07/2018 The mobility scholarship within the MayaNet Project-Erasmus Mundus Action 2

08/2012 - 08/2014 Stipend recipient within the master's degree program division from the National Council on Science and Technology of Mexico (CONACYT)

03/2013-09/2014 The scholarship for health research within the Research Development Division from the "Instituto Mexicano del Seguro Social (IMSS)"

02/2013 - 06/2014 Scholarship holder within the Institutional Program for 02/2011 - 12/2011 Training of Researchers (PIFI) from the "Instituto Politécnico Nacional (IPN)"

02/2005-12/2011 The Institutional scholarship from the "Instituto Politécnico Nacional (IPN)"

\subsection{Conference participations}

26th Annual meeting of the Society for Virology (GfV), Münster, Germany, 2016 Poster presentation: Importance of the GXXXA motif in the Ebolavirus GP transmembrane domain for entry and sensitivity towards host-cell encoded antiviral factors

National Symposium on Zoonoses Research, Berlin, Germany, 2016

Oral presentation: GXXXA motif within the transmembrane domain of the Ebola virus glycoprotein is important for counteraction of the antiviral host factor tetherin

Keystone Symposia on Hemorrhagic Fever Viruses, Santa Fe, New Mexico, USA, 2016

Poster presentation: The GXXXA motif within the GP transmembrane domain is important for Ebola virus counteraction of the antiviral host cell factor tetherin 
28th Annual meeting of the Society for Virology (GfV), Würzburg, Germany, 2018 Oral presentation: A GXXXA motif in the transmembrane domain of the Ebola virus glycoprotein is required for tetherin antagonism

National Symposium on Zoonoses Research, Berlin, Germany, 2018

Poster presentation: Ebola virus glycoprotein-driven entry requires cathepsin B/L activity irrespective of particle shape and target cell line

\subsection{List of publications}

González-Hernández, M., Hoffmann, M., Brinkmann, C., Nehls, J., Winkler, M., Schindler, M., Pöhlmann, S. 2018. A GXXXA motif in the transmembrane domain of the Ebola virus glycoprotein is required for tetherin antagonism. Journal of Virology pii: JVI.00403-18. doi: 10.1128/JVI.00403-18.

González-Hernández, M., Hoffmann, M., Pöhlmann, S. 2019. Calu-3 cells are largely resistant to entry driven by filovirus glycoproteins and the entry defect can be rescued by directed expression of DC-SIGN or cathepsin L. Virology (Submitted Manuscript)

Hoffmann, M., Crone, L., Dietzel, E., Paijo, J., González-Hernández, M., Nehlmeier, I., Kalinke, U., Becker, S., Pöhlmann, S. 2017. A polymorphism within the internal fusion loop of the Ebola virus glycoprotein modulates host cell entry. Journal of Virology 91: e00177-17. https://doi.org/ 10.1128/JVI.00177-17

Brinkmann, C., Nehlmeier, I., Walendy-Gnirß, K., Nehls, J., González-Hernández, M., Hoffmann, M., Qiu, X., Takada, A., Schindler, M., Pöhlmann, S. 2016. The tetherin antagonism of the Ebola virus glycoprotein requires an intact receptor-binding domain and can be blocked by GP1-specific antibodies. Journal of Virology 90:11075-11086. doi:10.1128/JVI.01563-16 
Hoffmann, M., González-Hernández, M., Berger, E., Marzi, A., Pöhlmann, S. 2016. The Glycoproteins of All Filovirus Species Use the Same Host Factors for Entry into Bat and Human Cells but Entry Efficiency Is Species Dependent. PLOS ONE 11(2): e0149651. doi:10.1371/journal.pone.0149651

Plegge, T.*, Spiegel, M.*, Krüger, N., Winkler, M., González-Hernández, M., Pöhlmann, S. 2019. Inhibitors of signal peptide peptidase and subtilisin/kexin-isozyme 1 inhibit Ebola virus glycoprotein-driven cell entry by interfering with activity and cellular localization of endosomal cathepsins. PLoS One (Submitted manuscript) *shared first authorship 